\title{
Dissecting Neuronal Circuits: Analysis of Basket Cell-like \\ Interneurons in Cortical Layer 6
}

\author{
Daniel Raphael Wyskiel
}

Roseville, Michigan

Bachelors of Science, Western Michigan University, 2006

A Dissertation presented to the Graduate Faculty of the University of Virginia in Candidacy for the Degree of Doctor of Philosophy

Neuroscience Graduate Program

University of Virginia

December, 2017 


\section{ABSTRACT}

To understand how neuronal circuits underlie the processing of brain function, methods must be employed to dissect the circuit. Identification of the neuronal subtypes and characterization of how they interact are required to effectivity gain insight into understanding the neuronal circuit. The method to decipher neuronal circuits I describe and use here is the simultaneous multiple patch-clamp recording system. My overall aim was to elucidate interneuronal circuits in cortical layer 6 (L6), which are critical for specific brain processes such regulating sensory information. Studies on the connectivity of L6 have predominately focused on excitatory pyramidal neurons. Relatively little is known of the subtypes of GABAergic interneurons in L6 and how they are incorporated into the circuit. However, the role of L6 GABAergic inhibitory interneurons in brain function has recently become more appreciated.

In this dissertation, I first provide background on L6 and basket cells, the most abundant GABAergic interneuron subtype. Then, in Chapters 2 and 3, I describe the simultaneous multiple patch-clamp recording system that is used to decipher interneuronal circuits. In Chapter 4, I examine a population of L6 interneurons using this method. Finally, I discuss my results and the field moving forward. 


\section{ACKNOWLEDGMENTS}

First and foremost, I thank my family. They have always been there for me. I also thank my advisor, Julius Zhu, and my thesis committee members, Alev Erisir, Ruth Stornetta, Douglas Bayliss, Michael Scott and Manoj Patel. Finally, I thank those in the Neuroscience Graduate Program and elsewhere at the University of Virginia who have helped me along the way. 


\section{CHAPTER 1}

\section{Introduction}

\section{Basket cells (BCs)}

Interneurons called "basket cells" were originally described by Ramón y Cajal in 1911 (Cajal, 1911). However, the pericellular "nests" thought to be formed by these cells which gave them their name were later found to be the result of multiple cells forming synapses around the postsynaptic cell (DeFelipe, Hendry, \& Jones, 1986; Marin-Padilla, 1969; Somogyi, Kisvarday, Martin, \& Whitteridge, 1983; Somogyi \& Soltesz, 1986; White \& Keller, 1989). Further studies showed that only a smaller portion of the boutons of BCs targeted the somas of pyramidal neurons, with the rest innervating their dendrites - either at dendritic shafts or spines (Di Cristo et al., 2004; Marin-Padilla, 1969; S, 1904; Szentágothai, 1973). Thus, the synapses made by BCs appear not in the form of "baskets," the characteristic that defined the subtype, and the majority of those synapses do not contact the soma.

\section{Identifying BCs}

The definition of a BC has clearly changed since it was first described. Currently, no definition is agreed upon. Different criteria are now used. However, while some researchers may use a criterion in classifying BCs, others use it in identifying putative BCs. A combination of methods is also sometimes employed. 
Identifying BCs or putative BCs at the macroscopic level has been used since Ramón y Cajal and is still used by some labs (Cajal, 1911; Jiang et al., 2015; X. Jiang, G. Wang, A. J. Lee, R. L. Stornetta, \& J. J. Zhu, 2013; E. H. Jones, SHC, 1984; Wang, Gupta, Toledo-Rodriguez, Wu, \& Markram, 2002). In general, this method examines the axonal arborization, and a description followed by many is stated here from Wang et al., 2002: "At the light microscopic level, short, bent axonal segments seemingly targeting neuronal somata have therefore been used as the general sign for classifying BCs" (Wang et al., 2002). However, many different descriptions have been used. The research team led by Julius Zhu has classified interneurons in L2/3 at the light microscope level and has described BCs as having "a basket-like axonal arborization" (X. Jiang et al., 2013; Wyskiel, Larry, Jiang, Wang, \& Zhu, 2016). While researchers using this method mainly focus on the axon, in some cases either the soma and/or the dendritic arborization has been used. This approach has drawbacks. Anatomical studies show that BCs are greatly diverse in both their axonal and dendritic arborizations (T. Freund, Martin, Smith, \& Somogyi, 1983; Kisvarday, Martin, Whitteridge, \& Somogyi, 1985; Kisvárday, 1992; Markram et al., 2004; Martin, Somogyi, \& Whitteridge, 1983; Somogyi et al., 1983; Thomson \& Lamy, 2007; Wang et al., 2002); and, their dendritic morphology can cause BCs to appear multipolar, bitufted or bipolar (Wang et al., 2002). Due to the great diversity in the spread of their axonal arbors of BCs, some researchers have divided BCs into subgroups, such as "large" and "small," with a third group termed "nests" more recently proposed (Gupta, Wang, \& Markram, 2000; Wang et al., 2002). The division of BCs based on morphology, however, has not been universally adopted (Xiaolong Jiang, Guangfu Wang, Alice J Lee, Ruth L Stornetta, \& J Julius Zhu, 2013; Wyskiel et al., 2016). 
Wang et al., 2002 acknowledged "that examination of synapses that target the soma should be included in the definition of a BC." While identifying axo-somatic synapses under the light microscope is used, verification at the electron microscopy (EM) level is thought to be necessary (Kubota et al., 2015). Identification of a certain portion of synapses made by BCs using EM is a method used by many for BC classification and some consider it the established criteria ( $\mathrm{T}$. Freund, Maglóczky, Soltesz, \& Somogyi, 1986; E. H. Jones, SHC, 1984; Kisvárday, 1992; Somogyi \& Soltesz, 1986). This portion varies by lab. Early studies using EM showed that about 20-30\% of the boutons targeted the soma of the postsynaptic cell (T. Freund et al., 1986;

Kisvarday, Martin, Somogyi, \& Friedlander, 1987; Kisvarday et al., 1985; Somogyi et al., 1983); however, later studies demonstrated significantly more variability with much lower percentages sometimes reported (Y Kawaguchi \& Kubota, 1998; Wang et al., 2002). Indeed, a BC may not form any of its synapses on the soma of a connected neuron (Kisvarday et al., 1987; Kubota et al., 2015; Tamás, Somogyi, \& Buhl, 1998; Wang et al., 2002). In addition, other interneuron subtypes can also form axo-somatic synapses and can even form "baskets" around postsynaptic somas (White \& Keller, 1989). Due to the difficulties in identifying axo-somatic synapses, some researchers have classified cells with that synapse near the soma as BCs or putative BCs. In this case, identifying BCs would appear to be a far departure from what began as an interneuron named for its formation of "baskets" around the somas of postsynaptic cells.

The use of electrophysiological firing patterns and genetic markers has also been used in identifying BCs (Tremblay, Lee, \& Rudy, 2016). However, not all BCs are FS and even those that can be labeled FS, display a range of firing patterns and are highly diverse in their passive membrane properties (Wang et al., 2002). In addition, other interneuron subtypes can also 
display FS properties. Nevertheless, BCs are often divided into subgroups based on their spiking and called FS-BCs and non-FS-BCs. Non-FS BCs are generally regular or burst spiking (Y Kawaguchi \& Kubota, 1998). Anatomically, both of these BC subgroups are highly diverse (Karube, Kubota, \& Kawaguchi, 2004).

Currently, BCs can be divided into different groups based on their expression of the calciumbinding protein parvalbumin (PV) or the neuropeptide cholecystokinin (CCK) (Yasuo Kawaguchi \& Kubota, 1997; Y Kawaguchi \& Kubota, 1998; Kubota \& Kawaguchi, 1997). These are termed PV-BCs and CCK-BCs. PV was found to be expressed by cells that morphologically resembled BCs and is now often used to label putative BCs. However, later it was shown that only a subset of BCs express PV. In addition, PV was found to be expressed in other interneuron subtypes (Pawelzik, Hughes, \& Thomson, 2002). Both PV-BCs and CCK-BCs are diverse in their firing and passive membrane properties. However, PV-BCs are largely FS cells, while CCK-BCs are generally regular-spiking cells (Tremblay et al., 2016). Anatomically, both PV-BCs and CCK-BCs are very diverse; however, PV-BCs typically correlate more with large and nest BCs than small BCs (Wang et al., 2002). Despite their overlapping morphologies, PV-BCs and CCK-BCs are thought to be distinct in their connectivity and function (Armstrong \& Soltesz, 2012; Bartos \& Elgueta, 2012; Tamás F Freund \& Katona, 2007; Tremblay et al., 2016).

\section{BCs in neuronal networks}


As shown above, BCs are composed of different subgroups that differ in their connectivity and function. However, the majority of BCs are those that express PV and are FS. These neurons represent a large portion of all interneurons in the brain - FS-BCs are thought to represent 40$50 \%$ of all interneurons in the brain (Kubota, 2014). In constituting such a large portion, it is not surprising that FS-BCs are have a critical role in many different brain functions and are thought to represent the main inhibitor in the neocortex (Rudy, Fishell, Lee, \& Hjerling-Leffler, 2011). By connecting on or near the soma, BCs more significantly control the action potential output of neurons and the timing of their spiking; and in doing so are thought to tightly regulate network activity (T. F. Freund \& Buzsáki, 1996; Tamás F Freund \& Katona, 2007). FS-BCs are thought to be responsible for establishing and maintaining fast cortical rhythms and in experiencedependent plasticity (Bartos, Vida, \& Jonas, 2007; Cardin et al., 2009; Hensch, 2005; Traub, Bibbig, LeBeau, Buhl, \& Whittington, 2004). In addition, FS-BCs are thought to be the main GABAergic interneuron regulating the balance between excitation and inhibition (Haider \& McCormick, 2009; Hasenstaub et al., 2005).

The role of FS-BCs in cortical functions is also dependent upon its intrinsic properties and its high rate of connectivity among pyramidal neurons. Due to their membrane and firing properties, FS-BCs receive fast synaptic inputs that induce fast action potentials. Other specializations allow FS-BCs to induce fast responses in the postsynaptic neuron. Thus, FS-BCs are responsible for fast, strong and reliable connectivity (Hu, Gan, \& Jonas, 2014; Tremblay et al., 2016). However, there is significant variability in the responses elicited by FS-BCs, which largely depends on whether the synapse is located on the soma or on the dendrites (Kubota et al., 2015). In addition, many factors can alter BC connectivity (Donato, Chowdhury, Lahr, \& Caroni, 2015; Donato, 
Rompani, \& Caroni, 2013; Lee et al., 2014; Xue, Atallah, \& Scanziani, 2014; Yoshimura, Dantzker, \& Callaway, 2005). For example, it can depend on differences in the activity of postsynaptic cells. This effect appears not to be the case in all interneuron subtypes (Xue et al., 2014). Thus, variability is also observed in neuronal connectivity, even among FS-BCs.

While BCs are very highly connected to pyramidal neurons, they are also extensively connected to each other, appearing to specifically target other BCs over other interneuron subtypes. In addition, BCs, in particular PV-BCs and FS-BCs, are also highly connected to each other via electrical synapses. This property is thought to be critical in specific brain functions (Gibson, Beierlein, \& Connors, 1999).

\section{Cortical Layer 6 (L6)}

\section{L6 Neurons}

L6, the deepest layer of the cortex, is present in all cortical areas. In general, it comprises nearly one third of the cortex, with some variation of thickness by region (Gabbott, Dickie, Vaid, Headlam, \& Bacon, 1997; Van Eden \& Uylings, 1985). L6 is distinct from other cortical layers in its cytoarchitecture. Due to its composition of cells, which vary greatly in size, shape and orientation, L6 is called the multiform or polymorph layer. Excitatory cells represent approximately $85-90 \%$ of the neurons in L6. These neurons include different subgroups of the classical pyramidal neurons (some with varying orientations), but also include those described as stellate cells and "star" pyramidal neurons, as well as other nonpyramidal glutamatergic neurons (Andjelic et al., 2009; Kaneko \& Mizuno, 1996; Mendizabal-Zubiaga, Reblet, \& Bueno-Lopez, 
2007; Tömböl, 1984). L6 pyramidal neurons are typically subdivided into two main subgroups based on whether their axons project to the thalamus (corticothalamic pyramidal neurons) or mostly within the cortex (cortico-cortical pyramidal neurons). These pyramidal neurons are most clearly distinct from those in L5 and L2/3 in that their apical dendrites typically do not ascend to and ramify within L1. However, a small subgroup of L6 pyramidal neurons (claustrumprojecting pyramidal neurons) do contain apical dendrites ascending to L1 (Katz, 1987). While some spare nonpyramidal glutamatergic neurons are found in other cortical layers, the great majority are found in L6 (Andjelic et al., 2009).

Attempts to identify and group L6 interneurons into subtypes began with the use of Golgi stain to examine the morphologies of so called local circuit neurons. Ramón y Cajal in 1911 described three subtypes: 1) cells with ascending axons, which include long ascending or Martinotti cells, 2) neurogliaform cells, and 3) local circuit neurons whose axons arborize in L6 (Cajal, 1911). In 1941, the L6 subtypes were expanded to five subtypes by O'Leary: 1) small basket cells, 2) neurons with horizontal axonal arborizations, 3) neurons with vertically oriented axonal arborizations that ramify in L4, 4) ascending non-Martinotti cells, and 5) Martinotti cells (O'Leary, 1941). Subsequent papers using either Golgi or Nissl staining further identified cell types in L6 based on their cell morphology alone, including a more recent study by Chen et al., 2009 (Chen, Abrams, Pinhas, \& Brumberg, 2009; Ferrer, Fabregues, \& Condom, 1986; Prieto \& Winer, 1999; Tömböl, 1984). Using whole-cell electrophysiological recordings and intracellular staining techniques, Kumar and Ohana, 2008 classified L6 interneurons in the barrel cortex of P19-22 rats into two subgroups: 1) L4-projecting and 2) local projecting interneurons (Kumar \& Ohana, 2008). Those projecting locally were described as both small and large BCs. The L4- 
projecting interneurons were thought to possibly include BCs as well. Another investigation using whole-cell electrophysiological recordings and intracellular staining techniques, The Blue Brain Project, spearheaded by Henry Markram, is examining postnatal days (P) 13-16 rats and has subdivided interneurons in all the cortical layers, including L6, into the classical defined morphological subtypes: 1) large BCs, 2) nest BCs, 3) small BCs, 4) chandelier cells, 5) Martinotti cells, 6) double bouquet cells, 7) bipolar cells, 8) bitufted cells, and 9) neurogliaform cells (Markram et al., 2015).

Some studies have shift away from classifying L6 interneurons based on the classically defined morphological subtypes, and the most recent published results use a different method entirely. Using a combination of approaches to examine electrophysiological, molecular and morphological properties, Perrenoud et al., 2012 classified L6 interneurons of P14-17 mice into 4 types: 1) interneurons that were fast spiking and expressed PV (51\%), 2) adapting interneurons transcribing SOM (26\%), 3) NPY-expressing cells resembling NGFCs (18\%), and 4) VIPexpressing GABAergic interneurons (5\%) (Perrenoud, Rossier, Geoffroy, Vitalis, \& Gallopin, 2012). PV-expressing cells were concentrated in the upper portion of L6 whereas those translating NPY were concentrated in the lower region. Notable, Perrenoud et al., 2012 stated that "we have not identified their morphological subclass in the current study." Most recently, a study by Arzt, Sakmann and Meyer, 2017 grouped interneurons based on their axon projections (Arzt, Sakmann, \& Meyer, 2017). In this study, five subgroups were described: 1) L6 inhibitors, 2) L6/5 inhibitors, 3) L5/6 inhibitors, 4) L5b inhibitors, and 5) L2/3/4 inhibitors. Despite a very in depth examination of their morphologies, including axon morphologies, Arzt, Sakmann and 
Meyer, 2017 did not identify their subgroups according to the classically defined morphological subtypes, such as BCs.

The complexities of L6 cytology described above are further exemplified by the discovery that L6 is divided into two distinct zones: L6a and L6b (Raedler \& Raedler, 1978). L6a abuts L5 and constitutes the great majority of the layer. L6b is a thin band lateral and superficial to the white matter. L6a and L6b are distinct in a number of ways, likely reflecting their dual origin (MarinPadilla, 1978)(Radnikow, Qi, \& Feldmeyer, 2015). Similar to cortical layers 2-5, L6a cells are derived from the cortical plate. L6b, however, contains cells derived from both the cortical plate and subplate. The structures of the two sublaminae differ in their arrangement and density of cells (O'Leary, 1941). L6a is denser in cell composition and contains pyramidal neurons, the principle excitatory neuron in the cortex. L6b contains a sparse composition of mostly horizontal cells. The composition of cells, including GABAergic interneurons, in L6a cells are thought to differ from those of L6b (Ferrer et al., 1986; Perrenoud et al., 2012).

\section{L6 neuronal circuitry}

L6 is a principal cortical output layer and is unique among cortical layers in regards to its connectivity to the thalamus. L6 receives direct inputs from and feedback to specific thalamic nuclei as well as providing output to other non-specific thalamic nuclei (Briggs, 2010; Lam \& Sherman, 2009; Radnikow et al., 2015; Thomson, 2010; West, Mercer, Kirchhecker, Morris, \& Thomson, 2005). This feedback to the thalamus is extensive and comprises nearly half of all the input to the thalamus (Erişir, Van Horn, \& Sherman, 1997; Guillery, 1969; E. Jones \& Powell, 
1969; Liu, Honda, \& Jones, 1995). L6 is also reciprocally connected to the claustrum, and has reciprocal connections within the cortex between other cortical layers and other cortical regions (Thomson \& Lamy, 2007). L6 connectivity to L4, the main thalamic input layer, is thought to further regulate thalamic connectivity to the cortex (Sherman, 2016). However, in contrast to L4, studies show that L6 is either unresponsive or exhibits very low activity in response to activity from thalamocortical cells, as shown for whisker deflection (Armstrong-James, Fox, \& DasGupta, 1992). In addition, L6 displays generally very low connectivity within the layer compared to the more highly connected L4 (Arzt et al., 2017; Beierlein \& Connors, 2002).

Studies on L6 connectivity have largely focused on pyramidal neurons. However, recently the role of interneurons in L6 function has been more clearly demonstrated (Olsen, Bortone, Adesnik, \& Scanziani, 2012)(Zhou et al., 2010). Those studies on L6 interneuron connectivity have predominately used genetic markers such as PV and somatostatin, but some of the cells may be FS-BCs (Bortone, Olsen, \& Scanziani, 2014; Cruikshank, Urabe, Nurmikko, \& Connors, 2010; Mercer et al., 2005; Oliva, Jiang, Lam, Smith, \& Swann, 2000). However, it should be noted that Arzt, Sakmann and Meyer, 2017 found no simple correlation between PV expression and axon projections. Some specificity in L6 connectivity has been uncovered, including connectivity to interneurons; for example, CT neurons preferentially connect to GABAergic interneurons over other CT neurons (Mercer et al., 2005; West et al., 2005; Zarrinpar \& Callaway, 2006). The Blue Brain Project is examining L6 connectivity using the classically defined morphological subtypes (Markram et al., 2015; Ramaswamy et al., 2015). However, no published papers on L6 connectivity from the Blue Brain Project can be found at this time. Some results can be seen using their online portal (Ramaswamy et al., 2015). 


\section{L6 BCs}

Caution should be used when reporting L6 BCs. Evidence suggests that at least some of the ways BCs are identified may not be employable in L6. Studies have compared what were described as BCs from different cortical layers and found significant differences between BCs residing in different layers; and based on these differences, the connectivity patterns established by BCs likely differ as well (Tremblay et al., 2016). In addition, anatomical descriptions of L6 BCs widely vary, as indicated by the studies described above and others (Karayannis, HuertaOcampo, \& Capogna, 2006; Kisvarday et al., 1987; Lund, Hawken, \& Parker, 1988; Markram et al., 2015; Tömböl, 1984). Furthermore, the axonal arborizations of other interneuron subtypes, while quite distinct from BCs in the superficial layers, may more closely resemble BCs in L6. Importantly, those cells described as BCs in the deeper layers make substantially fewer synapses onto somas compared to BCs in the superficial layers (Kisvárday, 1992).

Nevertheless, L6 contains BCs. Some studies have provided insight into L6 BC physiology (Beierlein \& Connors, 2002; Gibson et al., 1999; Kumar \& Ohana, 2008). Research examining the function of L6 BCs have focused primarily on PV-BCs, or on cells that may correspond to BCs such as FS interneurons and PV interneurons. Several of these studies suggest that L6 BCs may establish specific connectivity within L6 (Mercer et al., 2005; West et al., 2005). In addition, L6 FS interneurons were found to be strongly innervated by thalamocortical inputs and are thought to be responsible for rapid feed-forward inhibition (Cruikshank et al., 2010). These types of L6 interneurons were also found to be highly connected through electrical synapses (Gibson et al., 1999). 


\section{References}

Andjelic, S., Gallopin, T., Cauli, B., Hill, E. L., Roux, L., Badr, S., . . Lambolez, B. (2009). Glutamatergic nonpyramidal neurons from neocortical layer VI and their comparison with pyramidal and spiny stellate neurons. Journal of Neurophysiology, 101(2), 641-654.

Armstrong-James, M., Fox, K., \& Das-Gupta, A. (1992). Flow of excitation within rat barrel cortex on striking a single vibrissa. Journal of Neurophysiology, 68(4), 1345-1358.

Armstrong, C., \& Soltesz, I. (2012). Basket cell dichotomy in microcircuit function. The Journal of Physiology, 590(4), 683-694.

Arzt, M., Sakmann, B., \& Meyer, H. S. (2017). Anatomical Correlates of Local, Translaminar, and Transcolumnar Inhibition by Layer 6 GABAergic Interneurons in Somatosensory Cortex. Cerebral Cortex, 1-12.

Bartos, M., \& Elgueta, C. (2012). Functional characteristics of parvalbumin-and cholecystokininexpressing basket cells. The Journal of Physiology, 590(4), 669-681.

Bartos, M., Vida, I., \& Jonas, P. (2007). Synaptic mechanisms of synchronized gamma oscillations in inhibitory interneuron networks. Nature Reviews Neuroscience, 8(1), 4556.

Beierlein, M., \& Connors, B. W. (2002). Short-term dynamics of thalamocortical and intracortical synapses onto layer 6 neurons in neocortex. Journal of Neurophysiology, 88(4), 1924-1932.

Bortone, D. S., Olsen, S. R., \& Scanziani, M. (2014). Translaminar inhibitory cells recruited by layer 6 corticothalamic neurons suppress visual cortex. Neuron, 82(2), 474-485.

Briggs, F. (2010). Organizing principles of cortical layer 6. Frontiers in neural circuits, 4. 
Cajal, S. R. (1911). Histologie du syste me nerveux de I'Homme et des verte be s. Maloine (Paris), 2, 891-942.

Cardin, J. A., Carlén, M., Meletis, K., Knoblich, U., Zhang, F., Deisseroth, K., . . Moore, C. I. (2009). Driving fast-spiking cells induces gamma rhythm and controls sensory responses. Nature, 459(7247), 663-667.

Chen, C. C., Abrams, S., Pinhas, A., \& Brumberg, J. C. (2009). Morphological heterogeneity of layer VI neurons in mouse barrel cortex. Journal of Comparative Neurology, 512(6), 726-746.

Cruikshank, S. J., Urabe, H., Nurmikko, A. V., \& Connors, B. W. (2010). Pathway-specific feedforward circuits between thalamus and neocortex revealed by selective optical stimulation of axons. Neuron, 65(2), 230-245.

DeFelipe, J., Hendry, S., \& Jones, E. (1986). A correlative electron microscopic study of basket cells and large GABAergic neurons in the monkey sensory-motor cortex. Neuroscience, 17(4), 991-1009.

Di Cristo, G., Wu, C., Chattopadhyaya, B., Ango, F., Knott, G., Welker, E., . . Huang, Z. J. (2004). Subcellular domain-restricted GABAergic innervation in primary visual cortex in the absence of sensory and thalamic inputs. Nature neuroscience, 7(11), 1184.

Donato, F., Chowdhury, A., Lahr, M., \& Caroni, P. (2015). Early-and late-born parvalbumin basket cell subpopulations exhibiting distinct regulation and roles in learning. Neuron, 85(4), 770-786.

Donato, F., Rompani, S. B., \& Caroni, P. (2013). Parvalbumin-expressing basket-cell network plasticity induced by experience regulates adult learning. Nature, 504(7479), 272-276. 
Erişir, A., Van Horn, S. C., \& Sherman, S. M. (1997). Relative numbers of cortical and brainstem inputs to the lateral geniculate nucleus. Proceedings of the National Academy of Sciences, 94(4), 1517-1520.

Ferrer, I., Fabregues, I., \& Condom, E. (1986). A Golgi study of the sixth layer of the cerebral cortex. I. The lissencephalic brain of Rodentia, Lagomorpha, Insectivora and Chiroptera. Journal of anatomy, 145, 217.

Freund, T., Maglóczky, Z., Soltesz, I., \& Somogyi, P. (1986). Synaptic connections, axonal and dendritic patterns of neurons immunoreactive for cholecystokinin in the visual cortex of the cat. Neuroscience, 19(4), 1133-1159.

Freund, T., Martin, K., Smith, A., \& Somogyi, P. (1983). Glutamate decarboxylaseimmunoreactive terminals of Golgi-impregnated axoaxonic cells and of presumed basket cells in synaptic contact with pyramidal neurons of the cat's visual cortex. Journal of Comparative Neurology, 221(3), 263-278.

Freund, T. F., \& Buzsáki, G. (1996). Interneurons of the hippocampus. Hippocampus, 6(4), 347470. doi: 10.1002/(sici)1098-1063(1996)6:4<347::aid-hipo1>3.0.co;2-i

Freund, T. F., \& Katona, I. (2007). Perisomatic inhibition. Neuron, 56(1), 33-42.

Gabbott, P. L., Dickie, B. G., Vaid, R. R., Headlam, A. J., \& Bacon, S. J. (1997). Local-circuit neurones in the medial prefrontal cortex (areas 25, 32 and 24b) in the rat: morphology and quantitative distribution. Journal of Comparative Neurology, 377(4), 465-499.

Gibson, J. R., Beierlein, M., \& Connors, B. W. (1999). Two networks of electrically coupled inhibitory neurons in neocortex. Nature, 402(6757), 75.

Guillery, R. (1969). The organization of synaptic interconnections in the laminae of the dorsal lateral geniculate nucleus of the cat. Cell and Tissue Research, 96(1), 1-38. 
Gupta, A., Wang, Y., \& Markram, H. (2000). Organizing principles for a diversity of GABAergic interneurons and synapses in the neocortex. Science, 287(5451), 273-278.

Haider, B., \& McCormick, D. A. (2009). Rapid neocortical dynamics: cellular and network mechanisms. Neuron, 62(2), 171-189.

Hasenstaub, A., Shu, Y., Haider, B., Kraushaar, U., Duque, A., \& McCormick, D. A. (2005). Inhibitory postsynaptic potentials carry synchronized frequency information in active cortical networks. Neuron, 47(3), 423-435.

Hensch, T. K. (2005). Critical period plasticity in local cortical circuits. Nature Reviews Neuroscience, 6(11), 877-888.

Hu, H., Gan, J., \& Jonas, P. (2014). Fast-spiking, parvalbumin+ GABAergic interneurons: From cellular design to microcircuit function. Science, 345(6196), 1255263.

Jiang, X., Shen, S., Cadwell, C. R., Berens, P., Sinz, F., Ecker, A. S., . . Tolias, A. S. (2015). Principles of connectivity among morphologically defined cell types in adult neocortex. Science, 350(6264), aac9462.

Jiang, X., Wang, G., Lee, A. J., Stornetta, R. L., \& Zhu, J. J. (2013). The organization of two new cortical interneuronal circuits. Nature neuroscience, 16(2), 210-218.

Jones, E., \& Powell, T. (1969). An electron microscopic study of the mode of termination of cortico-thalamic fibres within the sensory relay nuclei of the thalamus. Proceedings of the Royal Society of London B: Biological Sciences, 172(1027), 173-185.

Jones, E. H., SHC. (1984). Basket cells. In Peters, A; Jones, EG. Cerebral cortex. Vol. 1 Cellular components of the cerebral cortex. 309-336.

Kaneko, T., \& Mizuno, N. (1996). Spiny stellate neurones in layer VI of the rat cerebral cortex. Neuroreport, 7(14), 2331-2336. 
Karayannis, T., Huerta-Ocampo, I., \& Capogna, M. (2006). GABAergic and pyramidal neurons of deep cortical layers directly receive and differently integrate callosal input. Cerebral Cortex, 17(5), 1213-1226.

Karube, F., Kubota, Y., \& Kawaguchi, Y. (2004). Axon branching and synaptic bouton phenotypes in GABAergic nonpyramidal cell subtypes. Journal of Neuroscience, 24(12), 2853-2865.

Katz, L. C. (1987). Local circuitry of identified projection neurons in cat visual cortex brain slices. Journal of Neuroscience, 7(4), 1223-1249.

Kawaguchi, Y., \& Kubota, Y. (1997). GABAergic cell subtypes and their synaptic connections in rat frontal cortex. Cerebral cortex (New York, NY: 1991), 7(6), 476-486.

Kawaguchi, Y., \& Kubota, Y. (1998). Neurochemical features and synaptic connections of large physiologically-identified GABAergic cells in the rat frontal cortex. Neuroscience, 85(3), $677-701$.

Kisvarday, Z., Martin, K., Somogyi, P., \& Friedlander, M. (1987). Evidence for interlaminar inhibitory circuits in the striate cortex of the cat. Journal of Comparative Neurology, 260(1), 1-19.

Kisvarday, Z., Martin, K., Whitteridge, D., \& Somogyi, P. (1985). Synaptic connections of intracellularly filled clutch cells: a type of small basket cell in the visual cortex of the cat. Journal of Comparative Neurology, 241(2), 111-137.

Kisvárday, Z. F. (1992). GABAergic networks of basket cells in the visual cortex. Progress in brain research, 90, 385-405.

Kubota, Y. (2014). Untangling GABAergic wiring in the cortical microcircuit. Current opinion in neurobiology, 26, 7-14. 
Kubota, Y., \& Kawaguchi, Y. (1997). Two distinct subgroups of cholecystokininimmunoreactive cortical interneurons. Brain research, 752(1), 175-183.

Kubota, Y., Kondo, S., Nomura, M., Hatada, S., Yamaguchi, N., Mohamed, A. A., . .

Kawaguchi, Y. (2015). Functional effects of distinct innervation styles of pyramidal cells by fast spiking cortical interneurons. elife, 4, e07919.

Kumar, P., \& Ohana, O. (2008). Inter-and intralaminar subcircuits of excitatory and inhibitory neurons in layer 6a of the rat barrel cortex. Journal of Neurophysiology, 100(4), 19091922.

Lam, Y.-W., \& Sherman, S. M. (2009). Functional organization of the somatosensory cortical layer 6 feedback to the thalamus. Cerebral Cortex, 20(1), 13-24.

Lee, S.-H., Marchionni, I., Bezaire, M., Varga, C., Danielson, N., Lovett-Barron, M., . . Soltesz, I. (2014). Parvalbumin-positive basket cells differentiate among hippocampal pyramidal cells. Neuron, 82(5), 1129-1144.

Liu, X. B., Honda, C., \& Jones, E. (1995). Distribution of four types of synapse on physiologically identified relay neurons in the ventral posterior thalamic nucleus of the cat. Journal of Comparative Neurology, 352(1), 69-91.

Lund, J. S., Hawken, M. J., \& Parker, A. J. (1988). Local circuit neurons of macaque monkey striate cortex: II. Neurons of laminae 5B and 6. Journal of Comparative Neurology, 276(1), 1-29.

Marin-Padilla, M. (1969). Origin of the pericellular baskets of the pyramidal cells of the human motor cortex: a Golgi study. Brain research, 14(3), 633-646. 
Markram, H., Muller, E., Ramaswamy, S., Reimann, M. W., Abdellah, M., Sanchez, C. A., . . . Arsever, S. (2015). Reconstruction and simulation of neocortical microcircuitry. Cell, 163(2), 456-492.

Markram, H., Toledo-Rodriguez, M., Wang, Y., Gupta, A., Silberberg, G., \& Wu, C. (2004). Interneurons of the neocortical inhibitory system. Nature reviews. Neuroscience, 5(10), 793.

Martin, K., Somogyi, P., \& Whitteridge, D. (1983). Physiological and morphological properties of identified basket cells in the cat's visual cortex. Experimental brain research, 50(2), 193-200.

Mendizabal-Zubiaga, J. L., Reblet, C., \& Bueno-Lopez, J. L. (2007). The underside of the cerebral cortex: layer V/VI spiny inverted neurons. Journal of anatomy, 211(2), 223-236.

Mercer, A., West, D. C., Morris, O. T., Kirchhecker, S., Kerkhoff, J. E., \& Thomson, A. M. (2005). Excitatory connections made by presynaptic cortico-cortical pyramidal cells in layer 6 of the neocortex. Cerebral Cortex, 15(10), 1485-1496.

O'Leary, J. L. (1941). Structure of the area striata of the cat. Journal of Comparative Neurology, 75(1), 131-164.

Oliva, A. A., Jiang, M., Lam, T., Smith, K. L., \& Swann, J. W. (2000). Novel hippocampal interneuronal subtypes identified using transgenic mice that express green fluorescent protein in GABAergic interneurons. Journal of Neuroscience, 20(9), 3354-3368.

Olsen, S. R., Bortone, D., Adesnik, H., \& Scanziani, M. (2012). Gain control by layer six in cortical circuits of vision. Nature, 483(7387), 47.

Pawelzik, H., Hughes, D. I., \& Thomson, A. M. (2002). Physiological and morphological diversity of immunocytochemically defined parvalbumin-and cholecystokinin-positive 
interneurones in CA1 of the adult rat hippocampus. Journal of Comparative Neurology, 443(4), 346-367.

Perrenoud, Q., Rossier, J., Geoffroy, H., Vitalis, T., \& Gallopin, T. (2012). Diversity of GABAergic interneurons in layer VIa and VIb of mouse barrel cortex. Cerebral Cortex, bhs032.

Prieto, J. J., \& Winer, J. A. (1999). Layer VI in cat primary auditory cortex: Golgi study and sublaminar origins of projection neurons. Journal of Comparative Neurology, 404(3), $332-358$.

Radnikow, G., Qi, G., \& Feldmeyer, D. (2015). Synaptic Microcircuits in the Barrel Cortex Sensorimotor Integration in the Whisker System (pp. 59-108): Springer.

Raedler, E., \& Raedler, A. (1978). Autoradiographic study of early neurogenesis in rat neocortex. Anatomy and embryology, 154(3), 267-284.

Ramaswamy, S., Courcol, J.-D., Abdellah, M., Adaszewski, S. R., Antille, N., Arsever, S., . . . Chalimourda, A. (2015). The neocortical microcircuit collaboration portal: a resource for rat somatosensory cortex. Frontiers in neural circuits, 9.

Rudy, B., Fishell, G., Lee, S., \& Hjerling-Leffler, J. (2011). Three groups of interneurons account for nearly $100 \%$ of neocortical GABAergic neurons. Developmental neurobiology, 71(1), 45-61.

S, R. y. C. (1904). Textura del Sistema Nervioso del Hombre y de los Vertebrados (Vol. 2): Madrid Nicolas Moya.

Sherman, S. M. (2016). Thalamus plays a central role in ongoing cortical functioning. Nature neuroscience, 19(4), 533-541. 
Somogyi, P., Kisvarday, Z., Martin, K., \& Whitteridge, D. (1983). Synaptic connections of morphologically identified and physiologically characterized large basket cells in the striate cortex of cat. Neuroscience, 10(2), 261-294.

Somogyi, P., \& Soltesz, I. (1986). Immunogold demonstration of GABA in synaptic terminals of intracellularly recorded, horseradish peroxidase-filled basket cells and clutch cells in the cat's visual cortex. Neuroscience, 19(4), 1051-1065.

Szentágothai, J. (1973). Synaptology of the visual cortex. Visual Centers in the Brain, 269-324.

Tamás, G., Somogyi, P., \& Buhl, E. H. (1998). Differentially interconnected networks of GABAergic interneurons in the visual cortex of the cat. Journal of Neuroscience, 18(11), $4255-4270$.

Thomson, A. M. (2010). Neocortical layer 6, a review. Frontiers in neuroanatomy, 4.

Thomson, A. M., \& Lamy, C. (2007). Functional maps of neocortical local circuitry. Frontiers in neuroscience, 1(1), 19.

Tömböl, T. (1984). Layer VI cells. Cerebral Cortex, 1, 479-519.

Traub, R. D., Bibbig, A., LeBeau, F. E., Buhl, E. H., \& Whittington, M. A. (2004). Cellular mechanisms of neuronal population oscillations in the hippocampus in vitro. Annu. Rev. Neurosci., 27, 247-278.

Tremblay, R., Lee, S., \& Rudy, B. (2016). GABAergic interneurons in the neocortex: from cellular properties to circuits. Neuron, 91(2), 260-292.

Van Eden, C., \& Uylings, H. (1985). Cytoarchitectonic development of the prefrontal cortex in the rat. Journal of Comparative Neurology, 241(3), 253-267. 
Wang, Y., Gupta, A., Toledo-Rodriguez, M., Wu, C. Z., \& Markram, H. (2002). Anatomical, physiological, molecular and circuit properties of nest basket cells in the developing somatosensory cortex. Cerebral Cortex, 12(4), 395-410.

West, D. C., Mercer, A., Kirchhecker, S., Morris, O. T., \& Thomson, A. M. (2005). Layer 6 cortico-thalamic pyramidal cells preferentially innervate interneurons and generate facilitating EPSPs. Cerebral Cortex, 16(2), 200-211.

White, E. L., \& Keller, A. (1989). Cortical circuits: synaptic organization of the cerebral cortex: structure, function, and theory: Birkhäuser Boston.

Wyskiel, D. R., Larry, T. C., Jiang, X., Wang, G., \& Zhu, J. J. (2016). Analysis of Transsynaptic Attentional Neuronal Circuits with Octuple Patch-Clamp Recordings. Advanced PatchClamp Analysis for Neuroscientists, 139-150.

Xue, M., Atallah, B. V., \& Scanziani, M. (2014). Equalizing excitation-inhibition ratios across visual cortical neurons. Nature, 511(7511), 596-600. doi: 10.1038/nature13321

Yoshimura, Y., Dantzker, J. L., \& Callaway, E. M. (2005). Excitatory cortical neurons form finescale functional networks. Nature, 433(7028), 868-873.

Zarrinpar, A., \& Callaway, E. M. (2006). Local connections to specific types of layer 6 neurons in the rat visual cortex. Journal of Neurophysiology, 95(3), 1751-1761. 


\title{
CHAPTER 2
}

\section{An optogenetics- and imaging-assisted simultaneous multiple patch-clamp recordings system for decoding complex neural circuits}

\author{
Guangfu Wang ${ }^{1,7}$, Daniel R. Wyskiel ${ }^{1,7}$, Weiguo Yang ${ }^{4}$, Yiqing Wang ${ }^{1,3}$, Lana C. Milbern ${ }^{1}$, \\ Txomin Lalanne ${ }^{1,5}$, Xiaolong Jiang ${ }^{1}$, Ying Shen ${ }^{6}$, Qian-Quan Sun ${ }^{4}$ and J. Julius Zhu ${ }^{1,2}$ \\ Departments of Pharmacology ${ }^{1}$, Neuroscience ${ }^{2}$ and Chemistry ${ }^{3}$, University of Virginia, \\ Charlottesville, VA 22908, USA
}

${ }^{4}$ Department of Zoology and Physiology, University of Wyoming, Laramie, WY 82071, USA

${ }^{5}$ Neuroscience Graduate Program, Department of Health and Life Sciences, Bordeaux University, 33076 Bordeaux Cedex, France

and

${ }^{6}$ Department of Neurobiology and Key Laboratory of Medical Neurobiology of Chinese Ministry of Health, Zhejiang University School of Medicine, 866 Yu Hang Tang Rd, Hangzhou 310058, China

${ }^{7}$ These authors contribute equally 
Deciphering neuronal circuitry is central to understanding brain function and dysfunction, yet it remains a daunting task. To facilitate the dissection of neuronal circuits, a process requiring functional analysis of synaptic connections and morphological identification of the interconnected neurons, I help present here a method for stable simultaneous octuple patchclamp recordings. This method allows physiological analysis of synaptic interconnections among 4-8 simultaneously recorded neurons and/or 10-30 sequentially recorded neurons, and provides insight to the anatomical identification of recorded interneurons and principal neurons. I also help describe the latest refinements and optimizations of mechanics, electronics, optics, and software programs central to the realization of a combined singleand two-photon microscopy-based optogenetics- and imaging-assisted stable simultaneous quadruple-duodecuple patch-clamp recordings system. Setting up the system, from the beginning of instrument assembly and software installation to full operation, can be completed in 3-4 days. 


\section{INTRODUCTION}

Modern electrophysiological tools have been employed in all aspects of neuroscience research due to their unparalleled high sensitivity and temporal resolution(Scanziani \& Hausser, 2009). The initial development of the simultaneous multiple (i.e., dual, triple and quadruple) patch-clamp recording method has greatly facilitated the investigation of communication between neurons and neuronal subcellular compartments in vitro and in vivo(Feldmeyer, Egger, Lubke, \& Sakmann, 1999; Larkum \& Zhu, 2002; Larkum, Zhu, \& Sakmann, 1999; Markram, Lubke, Frotscher, Roth, \& Sakmann, 1997; Reyes \& Sakmann, 1999; Schiller, Schiller, Stuart, \& Sakmann, 1997; Spruston, Schiller, Stuart, \& Sakmann, 1995; Stuart \& Sakmann, 1994; Waters, Larkum, Sakmann, \& Helmchen, 2003). However, deciphering complex interconnected neuronal circuits, a process requiring functional analysis of multi- and trans-synaptic connections and morphological identification of the cell types of many different interconnected neurons (Brown \& Hestrin, 2009a; Luo, Callaway, \& Svoboda, 2008; Wu, Tao, \& Zhang, 2011), remains a difficult endeavor. Because the testable connectivity pattern, $C$, increases exponentially as the number of simultaneously recorded neurons increases, $n$, or $C=4^{n \bullet(n-1) / 2}$, simultaneous patch-clamp recordings from four or more neurons exponentially increases the chance to decode and interrogate complex neuronal circuits. This principle was first verified by the Markram and Petersen groups who investigated the synaptic interconnections of excitatory neurons in the cortex with simultaneous hextuple-duodecuple patch-clamp recordings (Le Be \& Markram, 2006; Lefort, Tomm, Floyd Sarria, \& Petersen, 2009; Perin, Berger, \& Markram, 2011). Working with the engineers and technicians in commercial companies and local university workshops, the Julius Zhu lab has recently overcome various mechanical, electronic and software barriers to develop a 
stable simultaneous octuple patch-clamp recording technique that allows the recovery of the morphology of recorded excitatory neurons and recorded interneurons (Jiang, Wang, Lee, Stornetta, \& Zhu, 2013; A. J. Lee et al., 2014). The technique thus helps in the study and identification of inhibitory neurons, which frequently possess a dense intricate axonal arborization, in the complex neuronal circuits. With this technique, the Zhu lab has recently deciphered two transsynaptic disinhibitory and inhibitory neuronal circuits that span multiple layers and columns in the rat cortex (Jiang et al., 2013; A. J. Lee et al., 2014), further validating the advantage of making simultaneous patch-clamp recordings from four or more neurons. In this protocol, the Zhu lab describe the octuple patch-clamp recording technique, as well as the latest refinements and optimizations of mechanics, electronics, optics, and software programs that may allow the realization of an optogenetics- and imaging-assisted stable simultaneous multiple (from quadruple up to duodecuple) patch-clamp recordings system for functional interrogation of more complex neuronal circuits.

\section{Mechanics}

To accommodate eight or more motorized manipulators in a simultaneous multiple patch-clamp recording setup, compact manipulators are essential. Additionally, these manipulators need to be stable enough for long-lasting recordings ( $\geq 1-2 \mathrm{hrs})$ to be achieved. The Zhu lab found that it typically took $\sim 1-2$ hrs to thoroughly investigate all synaptic connections formed among 8 recorded neurons and to load a sufficient amount of cell marker (e.g., biocytin) to recover the 
complete cell morphology for identification of recorded neurons, particularly interneurons. In addition, stable manipulators allow easy replacement of new patch pipettes, and thus facilitate the sequential multiple patch-clamp recordings(Larkum \& Zhu, 2002).

A large variety of motorized manipulators are currently available on the market. The Zhu lab chose L\&N motorized manipulators (Luigs-Neumann GmbH, Ratingen, Germany) because over the years the company has been continuously improving the manipulators to keep up with our increasing demands in stability and compactness. The MINI series of L\&N manipulators were initially invented in 1992. These manipulators had their motor resolution refined to $9.8 \mathrm{~nm}$ per step and movement reproducibility improved to $<1 \mu \mathrm{m}$ when the Zhu lab started to build our simultaneous octuple patch-clamp recordings setup in 2008 (Fig 1a). The Zhu lab found that the MINI manipulators produced very smooth and highly reproducing movements, ideal for multiple stable recordings, and they were small enough to fit eight of them around a standard microscope. However, the Zhu lab soon realized that the mechanical disruption associated with the electrode replacement became a more significant issue with the increased number of neurons recorded. In response to our technical request and prototype suggestion for improvement, Luigs-Neumann $\mathrm{GmbH}$ developed multiple generations of adaptors for the electrode headstage in the following two years. Our tests showed that the $3^{\text {rd }}$ generation of adaptors, the guide rails, performed far better than the two older versions, the turning and backfolding adaptors. The guide rail exchange system allows the electrode holders to slide backward-and-forward along the manipulators to replace electrodes, largely eliminating the mechanical disruption associated with the turning or backfolding adaptors. Therefore, the system allows replacement of multiple patch pipettes 
multiple times to record many additional neurons without jeopardizing the existing recordings (Jiang et al., 2013; A. J. Lee et al., 2014).

To minimize the size of manipulators, Luigs-Neumann GmbH developed the smaller JUNIOR manipulator in 2002 (Fig 1b). However, the movement and stability of the first version of JUNIOR manipulators were not ideal. In 2010, the company completely redesigned the JUNIOR manipulator and the new JUNIOR manipulator has the motor resolution of $7.8 \mathrm{~nm}$ and reproducibility of $<1 \mu \mathrm{m}$. The Zhu lab used the new JUNIOR manipulators in simultaneous multiple patch-clamp recordings. The Zhu lab found that the JUNIOR manipulators had the movement resolution and stability comparable to the MINI manipulators. Therefore, both the MINI and JUNIOR L\&N manipulators are excellent choices for simultaneous multiple patchclamp recordings.

In 2013, Luigs-Neumann GmbH released a modified version of its JUNIOR manipulator, the JUNIOR COMPACT manipulator. The y-axis width of the manipulator was further minimized to $\sim 50 \mathrm{~mm}$, much smaller than the $\sim 100$-mm-wide JUNIOR manipulators or $\sim 150$-mm-wide MINI manipulators (Fig 1b). Our recent tests showed that the JUNIOR COMPACT manipulator had the same movement resolution and stability as the MINI and JUNIOR manipulates. The exceptionally small size of JUNIOR COMPACT manipulators makes it possible to accommodate 8-14 manipulators (octuple-quattuordecuple patch-clamp recordings) at a standard microscope or to realize a 20 patch (duodecuple patch-clamp recordings) system on a $360^{\circ}$ ring structure with a modified microscope. Moreover, the JUNIOR COMPACT manipulators alleviate the space 
competition among the instruments for electrophysiology, two-photon laser scanning imaging, and optogenetics. The reduction in space competition should be particularly significant for improving the simultaneous multiple in vivo patch-clamp recordings technology because two-photon imaging can improve the targeting of patch-clamp recordings (Kitamura, Judkewitz, Kano, Denk, \& Hausser, 2008; Komai, Denk, Osten, Brecht, \& Margrie, 2006) and optogenetics may help in the searching and investigating of synaptic connections (see below) in brain tissue slices and intact brains of anesthetized and behaving animals.

\section{Electronics}

Stable patch-clamp recordings have paved the way to effectively manipulate and detect neuronal activities, yet the manipulation and detection of neuronal activity are best achieved with highquality and low-noise amplifiers. Typically, one amplifier is needed for each recorded neuron. The Zhu labrecommend the Axoclamp 2A/B and Axopatch 200B amplifiers (Molecular Devices, Sunnyvale, CA) for voltage (current-clamp) and current (voltage-clamp) recordings, respectively, due to their unsurpassed low-noise performance and high compatibility with customizable operation software programs. Alternatively, one may choose the newer versions of Axon amplifiers, MultiClamp 700A/B. The MultiClamp 700A/B amplifiers are versatile amplifiers with two primary headstages, which are intended to support many electrophysiology applications, including voltage or current recordings from two neurons. These amplifiers are computercontrolled and they may only run under its designed program, the Axon MultiClamp Commander. 
To control the operation of an amplifier and to receive the experimental data collected by the amplifier, a computer is needed. To realize the communication between the multiple amplifiers and the computer, data acquisition interface boards with multiple digital-analog output (D/A) and analog-digital input (A/D) channels are preferred. In general, at least three channels are needed for operation control and data collection of an amplifier, with one D/A channel dedicated to its external command port and two A/D channels dedicated to its current and voltage output ports, respectively. Thus, for a simultaneous octuple patch-clamp recording setup, a data acquisition board with $8 \mathrm{D} / \mathrm{A}$ channels and $16 \mathrm{~A} / \mathrm{D}$ channels would be ideal. However, to the best of our knowledge, no such single interface board is commercially available. Therefore, in general, combining multiple interface boards is necessary to achieve simultaneous octuple or more patchclamp recordings (Fig 2). An InstruTECH ITC-18 data acquisition (DAQ) board (HEKA Instruments Inc., Bellmore, NY) has $4 \mathrm{D} / \mathrm{A}$ channels and $8 \mathrm{~A} / \mathrm{D}$ channels, which can thus support simultaneous recordings from 4 neurons. The electronics of ITC-18 board are optically isolated from the amplifiers and computer, ideal for the low-noise performance. The Zhu lab found it possible to use one computer to control multiple ITC-18 boards to realize operation and data collection of 8 or more amplifiers. In principal, the Zhu lab operated one ITC-18 board as the "master" board. When this "master" ITC-18 board started to send operation commands and acquire data, it also sent out a digital trigger signal to one or multiple other ITC-18 board(s) set to the external trigger mode. In this way, the computer could simultaneously control operation and data collection (both voltage and current data) of 8 or more amplifiers.

Alternatively, one may use DAQ boards manufactured by the National Instruments Corporation (Austin, TX). The National Instruments Corporation offers a large variety of DAQ boards (NI 
boards) with various numbers of $\mathrm{A} / \mathrm{D}$ and $\mathrm{D} / \mathrm{A}$ channels. Given the fast sample rate (up to 10 MHz), NI boards are perfect for imaging studies, yet they are also commonly used for electrophysiology recordings. Again, combining two or more NI boards is required to run simultaneous octuple or more patch-clamp recordings. As with ITC-18 boards, one may use the computer to activate one NI board and then use this NI board to trigger itself as well as other NI boards to control operation and data collection of 8 or more amplifiers. Combining ITC-18 and NI DAQ boards is also possible. The Zhu lab have verified that one computer can control multiple ITC-18 and NI boards to operate simultaneous electrophysiology recordings, two-photon laser scanning imaging and/or optogenetics.

The most recent versions of DAQ boards from the Molecular Devices are the Axon Digidata 1440 board that has $4 \mathrm{D} / \mathrm{A}$ channels and $16 \mathrm{~A} / \mathrm{D}$ channels, and the Axon Digidata 1550 board that has 8 D/A channels and 8 A/D channels. As with single ITC-18 and NI boards, one Axon Digidata 1440 board may run simultaneous dual-quadruple patch-clamp recordings because it can control the operation of up to 4 primary headstages of two MultiClamp amplifiers via 4 D/A channels. The Axon Digidata 1550 board can simultaneously support the operation and data collection of either voltage or current with up to four Axon MultiClamp amplifiers under Axon MultiClamp Commander. Therefore, the Axon Digidata 1550 board can run four Axon MultiClamp amplifiers to perform simultaneous octuple patch-clamp recordings collecting either voltage or current data. However, the Axon Digidata boards are not designed to operate in combination with other boards from the same or different companies. 


\section{Optics}

To perform optogenetics experiments, stimulation lasers can be delivered to the tissues via optical fibers and/or objective lenses. A modified optical fiber approach is the laserspritzer that can improve the spatial resolution of light illumination spots (see below). An alternative way to improve the spatial resolution is to combine single- and two-photon stimulation. The Zhu lab found that it was possible to steer the light beam of diode-pumped solid-state (DPSS) lasers into the light path of a two-photon laser scanning microscope. This enabled the optogenetic activation of neurons with single-photon laser pulse and/or two-photon laser scanning stimulations in the same experiments. The single- and two-photon laser focusing spots could be aligned in the same focal plane and controlled by single pair of scanning mirrors. To obtain the smallest laser spots, laser beams could be expanded by a telescope consisting of scanning and tube lenses to fill the entire rear aperture of the objective.

\section{Software}

IGOR Pro (WaveMetrics Inc., Portland, OR) is an interactive software environment that was chosen to carry out experiments and data analysis when the simultaneous dual, triple and quadruple patch-clamp recording techniques were initially developed. Over the years, many IGOR-based programs have been custom-written to handle the operation, online and offline data analysis of the experiments involved in multiple patch-clamp recordings and imaging applications. Since IGOR Pro can support multiple DAQ boards by the XOP file released by HEKA Instruments Inc., the Zhu lab upgraded an IGOR-based program used for quadruple patch-clamp recordings to support multiple ITC-18 boards and 8 or more amplifiers (Fig 3a). However, for ITC-18 boards running 
in the external trigger mode, the acquisition would not stop automatically even when the designated amount of data had been acquired. Thus, a stop command must be sent in time to prevent the FIFO memory of ITC-18 boards from overflowing. Moreover, the available sample interval setting in ITC-18 boards is related to the number of channels in operation. Therefore, to match the sample interval of multiple ITC-18 boards, one should ensure that the same number of channels in ITC-18 boards is operating the entire time. Finally, the sample rate of ITC-18 boards is too slow to support fast data acquisition, e.g., two-photon laser scanning imaging. To accommodate two-photon imaging, one may employ the NIDAQ Tools MX package that adds support for data acquisition directly into IGOR Pro. With the NIDAQ Tools MX package, the Zhu lab were able to use a single IGOR-based program to simultaneously run multiple NI boards to control two-photon laser scanning imaging (and optogenetics) in addition to ITC-18 boards that operate multiple patch-clamp recordings (Fig 3b-c).

Besides IGOR, MATLAB (Mathworks Inc., Natick, MA) is another widely used interactive software environment. MATLAB-based Ephus has been developed for cellular electrophysiology applications(Suter et al., 2010). The Zhu lab found that Ephus could run simultaneous dual recordings with its non-standard customized routines. The program is structured in a way that it can in principle support multiple recordings, including octuple patch-clamp recordings, but to the best of our knowledge this particular application has not yet been officially verified. Currently, the programmers of Ephus are working on a successor version of Ephus to include the routines that will make it easy for the application of Ephus in controlling octuple or more patch-clamp recordings. It is worth noting that Ephus can be easily linked with ScanImage, a co-evolved, powerful package dedicated for two-photon laser scanning microscopy(Pologruto, Sabatini, \& 
Svoboda, 2003). Adapting Ephus to run simultaneous octuple or more patch-clamp recordings should be an excellent alternative to satisfy the desire of combining electrophysiological recordings, two-photon laser scanning imaging and photostimulation.

It seems to be possible to use IGOR- and MATLAB-based software programs to communicate with Axon MultiClamp amplifiers using boards other than the Axon Digidata boards (e.g., ITC-18 and NI broads)(Brown \& Hestrin, 2009b; Buchanan et al., 2012; Couey et al., 2013; Xue, Atallah, \& Scanziani, 2014; Yu, Bultje, Wang, \& Shi, 2009). The approaches provide alternative solutions to run multiple Axon MultiClamp amplifiers to achieve simultaneous multiple patch-clamp recordings with two-photon laser scanning imaging and optogenetics.

\section{Experimental design}

While simultaneous multiple patch-clamp recordings are powerful in decoding complex neuronal circuits, the technique only works when all the components of the circuits (i.e., presynaptic neurons, postsynaptic neurons and their synaptic connections) are intact in the tissue preparations. Therefore, it is essential to have optimized brain slice preparations and high-quality patch-clamp recordings, which permit the relatively unbiased interrogation of the local neuronal circuits with their components located as far as $\sim 500-1,000 \mu \mathrm{m}$ apart(Jiang et al., 2013; Larkum \& Zhu, 2002; Le Be \& Markram, 2006; A. J. Lee et al., 2014; Lefort et al., 2009; Markram et al., 1997; Perin et al., 2011; Reyes \& Sakmann, 1999). To optimize the brain slice preparations, the Zhu lab recommend the use of a high-quality microslicer that can generate large-amplitude and highfrequency movements of the cutting blade in a horizontal axis with minimal vibrations in the 
vertical axis, which may confine tissue damage within $\sim 10 \mu \mathrm{m}$ below the cutting surface and thus produce brain slices with the best quality(Geiger et al., 2002). The Zhu lab also recommend cutting tissues at an angle closely parallel to the projections of dendrites and axons of neurons to minimize the truncation of selective populations of axons and dendrites(Davie et al., 2006; Jiang et al., 2013; A. J. Lee et al., 2014; J. J. Zhu, 2000). To achieve the high-quality recordings, the Zhu lab recommend use of the low-noise amplifiers and interfaces (see the above INTRODUCTION of electronics), proper arrangement of connecting electric circuits, and extensive practice of patchclamp recording skill prior to the actual experiments (see the below PROCEDURE). The averaged somatic whole-cell recording traces obtained from the high-quality recordings will have a solution to discern $\sim 10 \mu \mathrm{V}$ (in the current clamp mode) or $\sim 0.1 \mathrm{pA}$ (in the voltage clamp mode) events(Jiang et al., 2013; Le Be \& Markram, 2006; A. J. Lee et al., 2014; Lefort et al., 2009). Considering the conductance of AMPA and GABA receptor channels, as well as the dendritic filtering effects(J. J. Zhu, 2000), the smallest excitatory and inhibitory synaptic inputs from the distal dendrite would be $\sim 50 \mu \mathrm{V}$ or $\sim 0.5 \mathrm{pA}$ when they arrive at the soma. Thus, the high-quality patch clamp recordings should detect these smallest synaptic events, which has been experimentally verified (Jiang et al., 2013; Le Be \& Markram, 2006; A. J. Lee et al., 2014; Lefort et al., 2009). The Zhu lab do not recommend the use of a low or no sodium slicing solution as a substitute for a high-quality microslicer and/or a proper slicing procedure. This is because many superficial neurons, although they may survive the slicing process in the low or no sodium solution, have their dendrites and axons severely amputated. Recording from such neurons will interfere with achieving a more accurate measurement of synaptic connectivity and post hoc cell identification. Although programming the motorized manipulators to move patch pipettes to the positions just above the targeting tissue areas helps to speed up experiments (see below PROCEDURE), the Zhu labalso 
do not recommend the use of the automated patch clamp systems since there is currently no patchclamp algorithm designed to match the flexibility of a skilled patch-clamp experimenter. The Zhu lab noted that based on the slightly different conditions of individual neurons, skilled electrophysiologists are able to make the subtle modifications of parameters of all patch clamp steps (e.g., size of pipette, position to target pipette, amount of positive and sucking pressure applied, speed of sucking, and time to wait on sealing before break-in) to achieve high-quality recordings from every neurons.

Of course, preparing healthy brain slices containing intact presynaptic neurons, postsynaptic neurons and their connections, particularly when the long-distance circuits are examined, is not always possible. In these cases, or to further investigate the incoming axonal fibers originated from other brain areas or subareas, adding optogenetics to the procedure would be a solution (Petreanu, Huber, Sobczyk, \& Svoboda, 2007; Zhao et al., 2011). Furthermore, the multiple in vivo patch-clamp recordings technique, which can be combined with extracellular recordings(Constantinople \& Bruno, 2013), may be used to reveal and verify the key features of the organization of neuronal circuits(Jiang et al., 2013). As with the in vitro preparations, highquality animal preparation is essential since the injury, for example, at the cortical surface, could preclude high-quality recordings (and imaging) from cortical neurons and apical dendrites in the superficial layers in in vivo experiments(Jiang et al., 2013; Murayama \& Larkum, 2009; Tang, Brecht, \& Burgalossi, 2014; Y. Zhu \& Zhu, 2004). At the moment, the yield for detecting neuronal circuits with the multiple in vivo patch-clamp recordings method is fairly low (Jiang et al., 2013; Y. Zhu \& Zhu, 2004). However, the Zhu lab expect this to be improved when the two-photon imaging and/or optogenetics are combined to help search for the connections (see below). 


\section{Combination with other techniques}

The power of simultaneous multiple patch-clamp recordings in decoding complex neuronal circuits can be boosted when combined with electrophysiological, genetic, optogenetic and/or imaging monosynaptic connection "search" techniques in actual in vitro and in vivo experiments. The electrophysiological "search" technique was initially developed by Feldmeyer and his colleagues(Feldmeyer et al., 1999). This approach utilizes a relatively higher impendence (6-10 $\mathrm{M} \Omega)$ patch pipette as a "search" electrode to form a loose seal $(\sim 30-300 \mathrm{M} \Omega)$ on potential presynaptic neurons and elicit action potentials in the neurons with high intensity $(\sim 0.2-2 \mathrm{nA})$ short $(\sim 5-10 \mathrm{~ms})$ current pulses. When the current pulse stimulation induces coincident unitary excitatory or inhibitory postsynaptic potentials (UE/IPSPs) in recorded postsynaptic neurons, an indicator of putative synaptic connection, the "search" electrode will be repositioned to test other potential presynaptic neurons or removed. The normal patch pipettes will then move in to patch the putative presynaptic neurons and fully characterize the synaptic connections between these putative presynaptic and postsynaptic neurons. Given the improved stability associated with the updated electrode exchange/motorized manipulator system, the electrophysiological "search" techniques can be applied repetitively and intermittingly with simultaneous multiple patch-clamp recordings during individual experiments to reveal more complex (e.g., transsynaptic) neuronal circuits. 
The first genetic approach, invented by the Callaway lab, uses the rabies virus-based monotranssynaptic tracing technique(Osakada \& Callaway, 2013; Wickersham et al., 2007). The technique employs a modified rabies virus that can only retrogradely cross single synapses to label a small population of presynaptic neurons. This technique anatomically identifies a few monosynaptically connected neurons that can be used as the starting point for simultaneous multiple patch-clamp recordings to reveal more complex neuronal circuits involved in these and other neurons. The other genetic approach, developed by Kim and colleagues, is the GFP reconstitution across synaptic partners technique (GRASP)(Feng, Kwon, Lee, Oh, \& Kim, 2014; Kim et al., 2012). GRASP is based on the functional complementation between two nonfluorescent GFP fragments expressed at the presynaptic and postsynaptic neurons, respectively. If the nonfluorescent GFP fragments can be highly expressed only in single presynaptic or postsynaptic neurons within designated areas, GRASP may be potentially used as a way to identify the putative monosynaptically connected neurons although this idea has not yet been validated.

A recently invented optogenetic "search" technique employs a fine optic fiber-based laserspritzer to locally deliver laser light to activate presynaptic neurons(Sun, Wang, \& Yang, 2014). The "search" laserspritzer is manufactured by pulling the core optic fiber of a multi-mode fiber optic patch cable under a fire to generate a $\sim 5-10-\mu$ m-diameter tip (Fig $4 \mathrm{a}$ ). The optic fiber tip is then coated with the glass thawed from a patch pipette with a $\sim 30-50-\mu$ m-diameter tip (Fig $4 \mathrm{~b}$ ). With $0.1-0.8 \mathrm{~mW} / \mathrm{mm}^{2}$ laser power, the optic fiber tip produces a $\sim 10-30-\mu \mathrm{m}$-diameter light spot that can effectively activate single neurons expressing channelrhodopsin 2 (ChR2) and induce $\mathrm{uE} / \mathrm{IPSPs}$ in postsynaptic neurons (Fig 4c-d). With a laserspritzer, a large number of potential 
presynaptic neurons may be quickly scanned. Simultaneous multiple patch-clamp recordings can then be applied to make a full investigation of the putative and other potential synaptic connections.

The newest channelrhodopsin actuator, CheRiff, produces $\sim 2$-fold larger maximal photocurrent ( 2 nA) and has 9-fold increased photon sensitivity compared to ChR2(Hochbaum et al., 2014). This makes it possible to use high-magnification objective lenses (i.e., 40x or 60x) to focus the laser on the soma of CheRiff expressing neurons to elicit action potentials without applying the high laser illumination intensity that can injure neurons. Specifically, the Zhu labfound that to elicit action potentials in all L5 pyramidal neurons, the illumination intensity for ChR2 expressing neurons was larger than $2 \mathrm{~mW}$, whereas that for CheRiff expressing neurons was less than 200 $\mu \mathrm{W}$ (Fig 5a). Two-photon laser scanning stimulations provide a better spatial resolution of laser illumination spots than single-photon laser pulse stimulations. However, the Zhu labfound that even applying the various scanning techniques (e.g., spiral scanning, temporal focusing, lower NA objective, and their combinations) to increase the scanning area, two-photon laser scanning stimulations could only produce $\sim 200-400$ pA maximal photocurrent in CheRiff expressing L5 pyramidal neurons, which were not large enough to elicit action potentials in the majority of L5 neurons. This is consistent with the notion that two-photon laser scanning stimulations only stimulate a small fraction of the somatic membrane at the Z-axis compared to single-photon stimulations. Nevertheless, the increased CheRiff photocurrent makes it possible to combine single- and two-photon laser stimulations to increase the spatial resolution of laser activation areas. Our experiments showed that the combined single- and two-photon laser stimulation had increased half-height spatial resolution $(\sim 30 \mu \mathrm{m})$ compared to the single-photon stimulation $(\sim 60 \mu \mathrm{m})$ (Fig 5b-c). Using the combined single- and two-photon laser stimulation, the Zhu lab could search the 
putative presynaptic neurons and then confirm the synaptic connections with subsequent multiple patch-clamp recordings (Fig 5d). It should be pointed out that the search method was only effective in areas with sparse neurons expressing CheRiff because the Zhu lab frequently had falsepositive connections due to the activation of bypassing axons of other expressing neurons. Further improving the actuator construct to express CheRiff only in the soma and/or increase its photocurrent (permitting the use of more two-photon-dependent better spatial resolution photonstimulations) should make this an efficient search approach for identifying putative monosynaptic connections.

Finally, imaging techniques have also been used to identify monosynaptic connections. One early developed technique uses current injections to evoke a train of action potentials in "trigger" neurons while optically monitoring a large number of neurons loaded with calcium indicators to identify "follower" neurons(Kozloski, Hamzei-Sichani, \& Yuste, 2001). This method allows the detection of the putative postsynaptic neurons receiving strong facilitating excitatory synaptic connections that are large enough to produce action potentials in the "follower" neurons. To detect weaker excitatory synaptic connections or inhibitory synaptic connections typically observed in central neuronal circuits, scientists have since developed a large number of GFP-based and rhodopsin-based genetically encoded voltage indicators. The most recently developed archaerhodopsin-based voltage indicators, QuasArs and Archers, have the voltage sensitivity $\Delta \mathrm{F} / \mathrm{F}$ to be $\sim 30-90 \% / 100 \mathrm{mV}$, and can thus detect postsynaptic voltage responses of $\sim 1-5 \mathrm{mV}$, which enables the optical recording of single action potentials(Flytzanis et al., 2014; Hochbaum et al., 2014). However, QuasArs show rapid reduction in voltage sensitive after a few repetitive light illustrations (Hochbaum et al., 2014), whereas Archers have the slow response time of multiple 
milliseconds(Flytzanis et al., 2014). These imperfections hamper the use of the averaging strategy that permits the detection of smaller postsynaptic responses commonly found at neuronal connections.

In summary, these searching techniques can help identify anatomical or putative functional monosynaptic neuronal connections. However, the currently available search methods usually do not recover the cell morphology to unambiguously define the neuron identity. Moreover, both the spatial (e.g., selectively activating presynaptic neurons) and signal (e.g., detecting the common small postsynaptic responses) resolution of the optogenetics and imaging search methods remain to be improved (by $\sim 10-100$ folds) to be comparable to simultaneous multiple whole-cell recordings. Therefore, although the imaging and optogenetics methods can sometimes help to quickly pre-map the putative neuronal connections, at the moment, they serve more as an aid than a replacement of simultaneous multiple patch-clamp recording technique in deciphering complex neuronal circuits. In the other situations, for example, when the connectivity of neuronal circuits are investigated, directly applying the multiple patch-clamp recordings technique to randomly target all neurons in the entire area without referring any connectivity clues (e.g., those from the search techniques) would be a more accurate and productive approach(Jiang et al., 2013; A. J. Lee et al., 2014). 


\section{MATERIALS}

\section{REAGENTS}

○ Experimental animals: Rodents (neonatal, developing, or mature)

! CAUTION Animal experiments must conform to all relevant governmental and institutional regulations.

○ Carbogen (95\% oxygen, $5 \%$ carbon dioxide)

○ Sodium pentobarbital

- Artificial cerebrospinal fluid (ACSF; see REAGENT SETUP)

○ Biocytin (Sigma, cat. no. B4261-5MG)

○ $\mathrm{CaCl}_{2}$ (Sigma, cat. no. 223506-2.5KG)

- Cesium methanesulfonate $\left(\mathrm{CH}_{3} \mathrm{O}_{3} \mathrm{SCs}\right)$ (Sigma, cat. no. C1426-5G)

○ D-(+)-glucose (Dextrose, Sigma, cat. no. G8270-1KG)

○ EGTA (Sigma, cat. no. E3889)

○ HEPES (Fisher, cat. no. BP310-1)

○ $\mathrm{KCl}$ (Fisher, cat. no. BP366-1)

○ K-Gluconate (Sigma, cat. no. P1847)

○ $\mathrm{KOH}$ (Sigma, cat. no. 319376)

○ MgATP (Sigma, cat. no. A9187-100MG)

○ $\mathrm{MgCl}_{2}$ (Sigma, cat. no. 208337-1KG)

○ $\mathrm{Na}_{3} \mathrm{GTP}$ (Sigma, cat. no. G8877-10MG)

○ $\mathrm{NaH}_{2} \mathrm{PO}_{4}$ (Sigma, cat. no. S8282)

○ $\mathrm{NaHCO}_{3}$ (JT Baker, cat. no. JT3506-5)

○ Phosphocreatine disodium salt $\left(\mathrm{C}_{4} \mathrm{H}_{8} \mathrm{~N}_{3} \mathrm{O}_{5} \mathrm{PNa}\right.$, Sigma, cat. no. P7936-5G) 
- Double distilled water

\section{EQUIPMENT}

- Anti-vibration air table (e.g., Newport)

○ Dissection tools (e.g., Fine Science Tools Inc., scissors, forceps, scalpel, spatula)

○ High-quality vibrating tissue slicer (e.g., Ted Pella Inc., MicroslicerTM DTK-1000)

○ Razor blades for slicing (e.g., Gillette)

○ Cyanoacrylate glue (e.g., Krazy glue)

○ Filter paper (e.g., Fisher Scientific)

- Incubation chamber: ideally this should be a submerged chamber often in a glass beaker that allows for sufficient oxygenation of the slices during the recovery period along with dividers to keep the slices separate.

o Water bath

- Microscope

○ Water-immersion objective (40X)

- Intermediate magnification to achieve sufficient magnification

- Platinum ring covered with a grid of nylon strings

○ Heater

○ Osmometer (e.g., Wescor Inc., Vapro 5600)

○ Horizontal electrode puller (e.g., Sutter Instruments, Brown-Flaming P-87)

○ Stable motorized micromanipulators (see Introduction)

O Patch-clamp amplifiers (e.g., Axoclamp 2A/B and Axopatch 200B amplifiers, see INTRODUCTION)

○ Interface adapters (e.g., USB-18 and/or PCI-18, see INTRODUCTION) 
○ Computer interface (e.g., ITC-18 and/or NI BNC, see INTRODUCTION)

○ Video monitor

○ Software (e.g., IGOR Pro, see INTRODUCTION)

○ Thick-walled borosilicate glass (e.g., Sutter Instrument, cat. no. B200-116-10)

- 473 nm DPSS Laser (Coherent Inc., OBIS 473 LX)

○ Femtosecond Ti:Sapphire laser (Coherent Inc., Chameleon Ultra)

○ Fiber optic patch cable (Thorlabs Inc., cat. no. M38L02, Ø200 $\mu \mathrm{m}$ )

○ Scanning mirrors (Cambridge Technology, 6210H)

○ Shutters (Uniblitz, UHS1 and CS45)

○ Pockels Cell (Conoptics Inc., M350-80)

- Photodiode detector (Thorlabs, PDA100A)

○ Polarizing beamsplitter (e.g., Newport, 05FC16PB.5)

○ Mirrors (e.g., Newport, 10Z20ER.2)

○ Transmission electron microscope (e.g., EOL Ltd., JEOL-1230 transmission electron microscope)

\section{REAGENT SETUP}

Artificial cerebrospinal fluid (ACSF) $125 \mathrm{mM} \mathrm{NaCl}, 2.5 \mathrm{mM} \mathrm{KCl}, 1 \mathrm{mM} \mathrm{MgCl}, 2$ $\mathrm{mM} \mathrm{CaCl} 2,1.25 \mathrm{mM} \mathrm{NaH}_{2} \mathrm{PO}_{4}, 25 \mathrm{mM} \mathrm{NaHCO} 3$ and $25 \mathrm{mM}$ dextrose (pH 7.4). Use double distilled water. Measure the osmolarity using a vapor pressure osmometer. ACSF should be $\sim 300 \mathrm{mmol} / \mathrm{kg}$. Throughout the procedure, ACSF must be saturated with carbogen. ACSF should be stored at $4^{\circ} \mathrm{C}$ and used within 2-3 weeks. 
$\mathrm{MgCl}_{2}, 4 \mathrm{mM} \mathrm{Na} 2 \mathrm{ATP}, 0.4 \mathrm{mM} \mathrm{Na} 3 \mathrm{GTP}, 10 \mathrm{mM}$ disodium phosphocreatine, $0.6 \mathrm{mM}$ EGTA, $0.1 \mathrm{mM}$ spermine and $0.5 \%$ biocytin, for current recordings; $120 \mathrm{mM}$ potassium gluconate, 10 mM HEPES, 4 mM KCl, 4 mM MgATP, 0.3 mM Na 3 GTP, 10 mM disodium phosphocreatine and $0.5 \%$ biocytin, for voltage recordings $(\mathrm{pH} 7.25$, osmolarity $\sim 310$ $\mathrm{mmol} / \mathrm{kg}$ for both solutions). Intracellular solutions are made in $1 \mathrm{ml}$ aliquots and then stored at $-20^{\circ} \mathrm{C}$ for up to $6-12$ months.

Patch-pipette solution for "searching" in cell-attached mode Solution should be based on the intracellular solution (in case of accidental break through) but devoid of biocytin and very high potassium(Feldmeyer et al., 1999). This solution can also be made in $1 \mathrm{~mL}$ aliquots and stored at $-20^{\circ} \mathrm{C}$ for up to $6^{-}-12$ months.

\section{EQUIPMENT SETUP}

Patch-clamp setup (see INTRODUCTION) A description of the equipment needed for patch-clamp recording experiments can be found in Molecular Devices Axon Guide and previous publication(Davie et al., 2006).

Platinum ring Flattened platinum ring covered with a grid of nylon strings used for holding down the slice during recording(Edwards, Konnerth, Sakmann, \& Takahashi, 1989).

Laserspritzer Laserspritzer can be fabricated from a multi-mode fiber optic patch cable(Sun et al., 2014). The core optic fiber is exposed by stripping off the optic patch cable cladding, heated by a homemade syringe gas burner, gently pulled to make a tip size $\sim 5 \mu \mathrm{m}$ (Fig 4a). The pulled end of the optic fiber is inserted through a glass pipette with 
a $\sim 20-30-\mu \mathrm{m}$-diameter opening by $\sim 30-50 \mu \mathrm{m}$ under a microscope, and the tip of the glass pipette is then melted by a heat gun to seal with the optic fiber (Fig 4b).

Optical setup To enable single- and/or two-photon stimulation, the Zhu labcombined the beams of a $473 \mathrm{~nm}$ DPSS laser and a femtosecond Ti:Sapphire laser using a polarizing beamsplitter. The laser beams then were coupled into the light path of a custom-made twophoton laser scanning microscope. The power of femtosecond laser was controlled by a pockels cell. The dichroic mirrors in the microscope were used to aid the fine adjustment of DPSS laser intensity in the low power range. The pockels cell and/or Uniblitz shutters were used to control the laser pulse durations and a pair of scanning mirrors were used to control the position of laser spots. 


\section{PROCEDURE (Step by step methodology)}

\section{Hardware wiring •TIMING Approximately 4-12 h}

1) Mount data acquisition (DAQ) boards into computer.

For Instrutech ITC-18 DAQ boards, mount USB-18 or PCI-18 host interface adapters into a computer and connect them to ITC-18 interfaces.

For NI DAQ boards, mount boards into the computer by proper data bus and connect them to compatible BNC adapters.

2) According to your trigger strategy, wire all DAQ boards together.

3) Connect all patch-clamp amplifiers to DAQ interfaces, with BNC cables for current and voltage output ports connected to A/D ports, and external demand ports connected to D/A ports of DAQ interfaces (Fig 2).

4) Connect all headstages to the patch-clamp amplifiers.

5) Mount micromanipulators around recording chamber and adjust their positions.

6) Mount all headstages onto the micromanipulators.

7) Connect the micromanipulators to their controllers and control pads/wheels/joysticks. For L\&N manipulators, connect controllers together and set them as master or slave properly so that all manipulators can be controlled by a single SM7/8 pad.

ACRITICAL STEP: Proper arrangement and wiring of instruments reduces the noise level and thus decreases the number of recording traces needed to detect synaptic connections. 
8) Install drivers for DAQ boards

9) Install recording software, including IGOR Pro 6.0, XOP files for DAQ boards, and custom-written data acquisition and analysis programs.

-PAUSE POINT The operation of the system can be tested with model cells during this period. Once the system is working with model cells, it is ready to be used with acute brain slices.

\section{? TROUBLESHOOTING}

\section{Brain dissection and preparation of acute slices •TIMING Approximately 75-120 min}

10) Fill a $100 \mathrm{ml}$ beaker with ACSF and place it on ice with salt. Pour ice water around the stage of the microslicer. In a $250 \mathrm{ml}$ beaker, insert a submerged slice chamber with dividers. Fill the beaker with ACSF to just below the top of the chamber and place in a $37^{\circ} \mathrm{C}$ water bath. Bubble the ACSF in both beakers to saturation with carbogen.

ACRITICAL STEP: Allow sufficient time for ACSF to cool (until the ice in ACSF is visible) and saturate with carbogen.

-PAUSE POINT: Wait at least 15 minutes before proceeding to the next step.

11) Anesthetize the animal (e.g., with an intraperitoneal injection of sodium pentobarbital at $90 \mathrm{mg} / \mathrm{kg}$ of body weight).

! CAUTION Follow appropriate guidelines and regulations for animal experiments. 
12) Once deep anesthesia has been established, decapitate the animal with large scissors or a guillotine. Cut the skin along the midline to reveal the skull. Using small scissors cut the skull with slight upwards pressure. Peel the skull back with tweezers. Immediately pour icy ACSF over the brain. Using a scalpel, cut along the midline of the brain and extract the brain into the $100 \mathrm{ml}$ beaker filled with icy ACSF.

-PAUSE POINT: Allow the brain cool at this point. The time needed will depend on the size of the tissue block extracted.

13) Apply a thin layer of glue onto the platform in the chamber of the microslicer. Use a spatula to transfer the brain onto a piece of filter paper to remove excess ACSF. Make sure the midline of the brain is sufficiently dry.

14) Gently place the brain on the platform in the cutting chamber. After the brain is sufficiently glued in place, submerge the brain in the microslicer chamber with icy ACSF. ACRITICAL STEP: The time from decapitation to submersion of the brain in icy ACSF, along with the time the brain is out of solution, must be kept to a minimum.

\section{? TROUBLESHOOTING}

15) Begin slicing the brain. After removing a small initial section to examine the region of interest, cut slices at $\sim 300-400 \mu \mathrm{m}$. Ensure that the speed of the slicer, along with the 
vibration amplitude and frequency, are optimal for slicing without compressing the brain. The optimal settings will depend on the type of slicer used.

$\triangle$ CRITICAL STEP: Optimizing the slicing settings will facilitate the production of the highest quality tissue slices. The slicing speed should be lower for both the softer neonatal and harder adult and aged brain tissues(J. J. Zhu, 2000).

\section{? TROUBLESHOOTING}

16) Transfer slices to the incubated chamber after each slice is obtained. Keep track of the order of each slice. Using a partition helps keep the slices separated and ordered. Incubate slices in oxygenated ACSF for $30-60$ min at $37 \pm 0.5^{\circ} \mathrm{C}$.

\section{Visualization and determining the optimal slice •TIMING Approximately 15-30 min}

17) Transfer a cortical brain slice to the recording chamber on the stage of an upright microscope. The recording bath should be continuously perfused with oxygenated ACSF and held at $34.0 \pm 0.5^{\circ} \mathrm{C}$. Orient the brain to help identify neurons from distinct layers. Hold the slice in place on the chamber by placing a platinum ring covered with a grid of nylon strings on top of the slice.

\section{? TROUBLESHOOTING}

18) Using a $40 \mathrm{X}$ water-immersion lens with a $2 \mathrm{X}$ magnifier, assess the overall health of the tissue and cells. Identify a layer 5 (L5) pyramidal neuron in the cortex and visually track its apical dendrite towards L1. 
A CRITICAL STEP: In an ideal slice, the entire apical dendrites of many L5 pyramidal neurons can be visually tracked with minimal focusing through the slice. Typically, only one or two slices per animal meet this requirement.

\section{? TROUBLESHOOTING}

\section{Octuple patch-clamping •TIMING Approximately 30-45 min ( 4-5 min per cell)}

19) Once the ideal slice has been chosen, examine the area of interest for cells. Identifying healthy cells and then determining which electrode would best patch which cell will help expedite the patching process. When search techniques are used, the Zhu labrecommend capturing transmitted light images of the cells in the area with the landmarks to help to find the putatively connected cells (cf. (Le Be \& Markram, 2006)).

\section{? TROUBLESHOOTING}

20) Using a needle and filter, fill a glass electrode with enough intracellular solution to cover the electrode wire. Insert the electrode into the pipette holder and firmly seal the holder.

21) Apply positive pressure ( 20-60 mbar) to the pipette holder chamber via the connected tubing attached to the side of the pipette holder. Maintain the pressure by closing a valve. Repeat this step for all electrodes.

22) Move a pipette into the bath and underneath the objective. Make sure the tip of the pipette is not clogged and there is a steady stream of ACSF from the tip.

23) Decrease the positive pressure until there is a very small stream of ACSF out of the tip. This will reduce the chances of clogging the pipette and decrease the amount of highpotassium solution flowing onto the slice. 
24) With the electrode in voltage clamp, apply a voltage step (e.g., $1 \mathrm{mV}, 10 \mathrm{~ms}$ square pulse) to determine the current deflection. Using Ohm's law, the resistance of the pipette tip can be calculated $(R=V / I)$. This resistance gives a good indication of the tip size and can verify that the pipette is unblocked. The resistance of the pipette should be between 3-7 $\mathrm{M} \Omega$.

25) Lower the pipette towards the slice.

26) Repeat steps 20-23 for the remaining electrodes. Using the preinstalled or custom-written program to control the motorized manipulators in the steps can be time-saving. Note using the automation increases the risk of the electrodes colliding with each other or with the slice and objective.

27) Zero any voltage offsets.

28) Move the first pipette into position and increase the positive pressure. Lower the pipette into the slice. Ensure there is adequate positive pressure to blow debris away from the tip. ACRITICAL STEP: Cleaning off the debris surrounding the targeting cell is a prerequisite for a high-quality patch recording(Edwards et al., 1989). The exact amount of positive pressure needed, ranging from $\sim 20-60$ mbar, will depend on many factors, including the electrode tip size, depth of the targeting cells, and tissue health. Application of proper pressure can be crucial for cleaning off the debris and achieving $>1 \mathrm{G} \Omega(\sim 10 \mathrm{G} \Omega$ would be ideal) seal patch-clamp without interfering with other recordings. The Zhu lab recommend sufficient patch-clamp recording practice prior to the actual experiments.

29) Slowly advance the electrode towards the cell. An increase in resistance (seen in the test pulse), along with the appearance of a dimple on the cell, should occur as the tip approaches 
the neuron. At this point, release the positive pressure and apply negative pressure to the electrode. Clamp to a negative potential $(\sim-70 \mathrm{mV})$ to facilitate the formation of the highresistance seal. Release the negative pressure and withdraw the electrode slightly if the pipette has advanced too far into the cell. If a tight $\mathrm{G} \Omega$ seal has formed, apply pulses of negative pressure to break into the cell bringing the electrode to a whole-cell patch configuration with access to the intracellular milieu.

30) Repeat steps 25-27 with the remaining electrodes. At this point different strategies can be employed, such as searching for connected cells with the techniques described above. A“searching" pipette can typically be used to test several ( $\sim 5-10)$ presynaptic neurons. However, it should be exchanged once a loose-seal cannot be established. As with the patching electrodes, the amount of positive pressure ( 20-60 mbar) needed for "searching" electrodes should be just enough to clear away debris in front of the electrode but not disturb the other electrodes.

ACRITICAL STEP: High-quality patch recordings with minimal damage of neurites in the recording area help to achieve a more accurate neuronal connectivity measurement and cell identification. Multiple attempts and side movements of electrodes should be avoided when approaching the targeted neurons. Proper training and sufficient practice of animal/tissue preparation and patch recording skills prior to the actual experiments can be extremely beneficial (Chen et al., 2012; Larkum \& Zhu, 2002; Y. Zhu \& Zhu, 2004).

Identifying and examining synaptic connections •TIMING Approximately 60-240 min ( 15-60 min per two cells) 
31) Place the cells into current-clamp mode. Inject a depolarizing current step ( $\sim 10 \mathrm{~ms})$ into one of the cells to reliably induce an action potential in the presynaptic neuron. To speed up the investigation, an alternative approach is to inject a depolarizing current step into one of the cells, and another current step into another cell at $\sim 1 \mathrm{sec}$ later in the tests. Monitor the current or voltage of the other cells for a postsynaptic response.

\section{? TROUBLESHOOTING}

32) Record 50-250 sweeps. The amount of sweeps necessary to establish a postsynaptic response will depend on the strength of the response and the noise level(Debanne et al., 2008).

\section{? TROUBLESHOOTING}

33) After characterizing the physiology and allowing sufficient time for filling the neurons with biocytin, slowly withdraw a pipette (ideally you should be able to see the membrane stretch away from the cell). Once the pipette is $\sim 5-10$ microns away, retract the pipette rapidly out of the recording chamber.

$\triangle$ CRITICAL STEP: Depending on the extent of the dendritic tree and axon, recordings must last at least 15-30 minutes to allow for the sufficient diffusion of biocytin into the neuron. The Zhu lab suppose that the failure to reseal the membrane after the electrode removal to be the primary contributor to loss or incomplete recovery of the morphology of the small percentage of cells.

\section{? TROUBLESHOOTING}


34) Fix the slice (lying flat) in $3 \%$ acrolein $/ 4 \%$ paraformaldehyde in $0.1 \mathrm{M}$ phosphate-buffered saline at $4^{\circ} \mathrm{C}$ for $24-48$ hours.

-PAUSE POINT Fixed tissues can be kept at $4^{\circ} \mathrm{C}$ for up to $\sim 2-3$ weeks.

$\triangle$ CRITICAL STEP: Including acrolein in the fixation solution helps to preserve the ultrastructure of the brain slice tissues.

! CAUTION Acrolein and paraformaldehyde are toxic and/or carcinogen. They should be handled according to the approved institutional biosafety protocol.

\section{Morphological reconstruction •TIMING Approximately 2-7 d}

35) After the 24-48 hour incubation period, process the slice using the avidin-biotinperoxidase method to elucidate the morphologies of the recorded cells. An example protocol can be found in a previous publication(Marx, Gunter, Hucko, Radnikow, \& Feldmeyer, 2012).

$\triangle$ CRITICAL STEP: If the ultrastructure examination is planned to validate synaptic connections, overreaction of avidin-biotin-peroxidase should be avoided since it prevents visualization of presynaptic and postsynaptic ultrastructure. The fixation and immunostaining processing may need to be modified if the investigator is interested in further examining the neuronal connections of recorded and other neurons with the CLARITY procedure(Tomer, Ye, Hsueh, \& Deisseroth, 2014).

\section{? TROUBLESHOOTING}

36) Use a computerized reconstruction system, such as Neurolucida (MBF Bioscience, Williston, VT), to reconstruct the recorded neurons. 


\section{Electron microscopic examination •TIMING Approximately 4-8 wk}

37) Re-section the slice into $60 \mu \mathrm{m}$ sections and postfix in $1 \% \mathrm{OsO}_{4}$.

38) Counterstain the sections with $1 \%$ uranyl acetate.

39) Choose areas of interest $(\sim 50 \mathrm{x} \sim 50 \mu \mathrm{m})$ that contain putative synaptic boutons from single synaptic neurons. Flat embed the sections in resin.

40) Carefully excise and then resection into $80 \mathrm{~nm}$ ultrathin sections with an ultramicrotome. Do not excise and resection if the synaptic boutons originating from different presynaptic neurons are too close to separate.

41) Examine the ultrathin sections in sequence by following the labeled dendrites using a transmission electron microscope. These will typically lead to all microscopic synapses except those very few synapses either destroyed during electron microscopic processing or hidden behind grids. The order in which each synapse is identified should be predicted by the Neurolucida reconstruction.

$\triangle$ CRITICAL STEP: Because it is not possible to unambiguously identify the origins of the presynaptic terminals under an electron microscope, it is important not to include the synaptic boutons originating from different presynaptic neurons in the same ultrathin sections. In addition, reconstructing the presynaptic and postsynaptic neurons and marking their putative contact sites with Neurolucida, and examining the ultrathin sections in sequence, will facilitate the recovery of synapses under the electron microscope.

\section{Table 1 | Troubleshooting table.}




\begin{tabular}{llll}
\hline Step & Problem & Possible reason & Solution \\
\hline 7 & Lack of & Cables are not connected & Connect the cables correctly (see \\
& signal/command & correctly & Figure 2) \\
& operation & & \\
& & & \\
$8-9$ & Software will not & Program is not installed & Check that the program is installed \\
& coad/acquire & correctly & correctly \\
& & \\
data/execute & & \\
commands &
\end{tabular}

13-17 Cannot identify slice Did not cut slice at correct Make sure brain tissue is flat on containing dendrites angle the ramp parallel to plane of slice/ cannot trace L5 apical dendrite to $\mathrm{L} 1$ is disrupting the slice

Slice is unhealthy

Optics are not optimal Moving pipette within slice
Follow the correct slicing procedure

Optimize the optics(Davie et al., 2006)

$29 \quad$ Losing cell after patching another 
Pipette tip has drifted from Move previously patched

cell

electrodes back to their original

location on cell if it has moved

Too much positive pressure Adjust the pressure to the pipette

Slice is not healthy

Correct slicing procedures must be

followed

Slice does not contain

parallel dendrites

Make sure that the pyramidal

apical dendrites run parallel to the

surface of the slice

Cells are too superficia

and connections are

severed

Cells patched do not

Try searching for connections

connect in situ

The holding potential of

Modify internal and holding

the postsynaptic neuron is potential 
too near the reversal

potential

Stimulate 3 or more action

Release probability is too

potentials

low; axon failure

Reduce the noise level

Noise level is too high

31-33 Morphology is not recovered

Cell dies while recording

The cell must be healthy when retracting the electrode

Cell membrane breaks

down when electrode is removed from cell

Problem with the biocytin

filling and/or labeling

procedure
Follow the correct biocytin

labeling and diaminobenzidinebased procedures(Marx et al., 2012) 


\section{•TIMING}

Steps 1-7, hardware wiring: 4-12 h

Steps 8-9, software installation: 4-12 h

Steps 10-14, brain dissection and preparation of acute slices: $75-120 \mathrm{~min}$

Steps 15-16, visualization and determining the optimal slice: $15-30 \mathrm{~min}$

Steps 17-28, octuple patch-clamping: 30-45 $\mathrm{min}$

Steps 29-32, identifying and examining synaptic connections: $1-4 \mathrm{~h}$

Steps 33-34, morphological reconstruction: 2-7 d

Steps 35-39, electron microscopic examination: 4-8 wk 


\section{ANTICIPATED RESULTS}

With a stable octuple recordings system properly set up as described above, simultaneous recording from 8 neurons is fundamentally similar to recordings from one or two neurons. A skilled electrophysiologist can expect to master the technique and make it as a routine within $\sim 1$ month. Afterward, the investigator can typically achieve 6-8 successfully patched neurons per slice on the first attempt. Then, the investigator may decide if it is necessary to replace the failed pipette(s) to get the missed/new neurons. The success rate of patching additional neurons by replacing pipettes without losing those already recorded is $\sim 90 \%$. These recordings of $6-8$ neurons usually last for 2-4 hrs, permitting the full investigation of all synaptic connections and aid in cell identification. Compared to the previous experiments(Larkum \& Zhu, 2002; J. J. Zhu, 2000), both the success rate of re-patching neurons and the recording time of high-quality in vitro and in vivo recordings are significantly enhanced(Jiang et al., 2013; A. J. Lee et al., 2014). The Zhu lab attribute these achievements primarily to the recently improved mechanical stability of the L\&N manipulators and associated guide rail electrode exchange systems. The expected yield for the number of synaptically connected neurons will depend on the actual connectivity of recorded neurons, as well as other factors, such as the quality of the preparations and whether the searching techniques outlined above are performed.

Simultaneous patch-clamp recording from 4 or more neurons allow the examination of complex neuronal circuit patterns (e.g., reciprocal, transitive, circular, parallel, converging and/or diverging circuits and their combinations) that are involved in multiple excitatory and inhibitory neurons, which seem to be common in the brain(Jiang et al., 2013; Le Be \& Markram, 2006; A. J. Lee et al., 2014; Lefort et al., 2009; Perin et al., 2011). Although the Zhu lab focus on the setup of 
simultaneous octuple patch-clamp recordings and exemplify applications in rat cortical brain slices in this protocol, the Zhu lab anticipate that the approach can be adapted to set up other simultaneous multiple (quadruple-duodecuple) patch-clamp recordings systems as well. Moreover, the technology can be employed to investigate the functional organization of neuronal circuits in many different brain regions across a broad range of species (from mice to monkeys) at all developmental ages in vitro and in vivo. For the wide range of neonatal, developing and adult animal in vitro and in vivo preparations, the Zhu lab refer to other previous publications and protocols(Chen et al., 2012; Davie et al., 2006; Jiang et al., 2013; Kitamura et al., 2008; Komai et al., 2006; A. K. Lee, Epsztein, \& Brecht, 2009; Stern, Maravall, \& Svoboda, 2001; J. J. Zhu, 2000; J. J. Zhu \& Connors, 1999; Y. Zhu, Stornetta, \& Zhu, 2004). As discussed above, multiple patch-clamp recordings with two-photon laser scanning microscopy and optogenetics is instrumentally possible; together they would work complementarily or synergistically in the investigation of complex local and longrange neuronal circuits.

\section{ACKNOWLEDGEMENTS}

The Zhu lab thank Drs. Rodrigo Andrade, Mark Beenhakker, Toni Figl, Vijay Iyer, Jinny Kim, Peter Neumann, Karel Svoboda for technical advice and invaluable discussions, and members of the Zhu laboratory for comments and technical assistance. While the Zhu lab discuss here a few approaches to set up a simultaneous multiple patch-clamp recordings, two-photon laser scanning and optogenetics system using the mechanics, electronics, optics and software that the Zhu lab have some experience with, the Zhu lab anticipate alternative approaches to achieve the same goal 
with other instruments and software programs. The Zhu lab hopes that this protocol will inspire more scientists to contribute their wisdom and experience to this subject. 


\section{FIGURE LEGENDS}

Figure 1. Manipulators for simultaneous multiple patch-clamp recordings.

(a) Photograph of an array of eight MINI L\&N motorized manipulators at a standard microscope.

(b) Photographs of the side (b1) and top (b2) views of a 151-mm W x 151-mm D x 197-mm H MINI L\&N motorized manipulator first invented in 1992 (left), a 104-mm W x 114-mm D x 130mm H JUNIOR L\&N motorized manipulator redesigned in 2010 (middle), and a 49-mm W x 114mm D x 157-mm H JUNIOR Compact L\&N motorized manipulator developed in 2013 (right) on a 25 -mm-grid breadboard. Note $\sim 50 \mathrm{~mm}$ width of the JUNIOR Compact manipulator in the $\mathrm{y}$ axis. Note that the red adaptors can be custom-removed to further reduce the width of the manipulators. 
Figure 2. Hardware wiring for simultaneous multiple patch-clamp recordings.

Schematic sketch shows the hardware wiring of the computer, interfaces and manipulators for the simultaneous multiple ( $\geq$ octuple) patch-clamp recordings setup. Inset image shows arrangement of the eight patch pipettes and recording chamber. 
Figure 3. IGOR-based program for simultaneous multiple patch-clamp recordings.

(a) Screenshot of IGOR-based program testing synaptic connections formed among eight recorded neurons.

(b) Screenshot of IGOR-based program measuring calcium transients at synapses of two neurons. Note that IGOR-based program displayed on multiple monitors can simultaneously run electrophysiology, two-photon laser scanning imaging and/or optogenetics routines.

(c) Flowchart showing generic sequence of operations during experiments. The laser light for optogenetic stimulation is delivered through the objective of the microscope (when no image data acquisition is required) and/or an optic fiber. 


\section{Figure 4. Laserspritzer-based synaptic connection "search" technique.}

(a) Schematic graph shows the fabrication of laserspritzer fiber probe.

(b) The tip of a laserspritzer (b1) and light spot produced by the laserspritzer with laser illumination (b2) under a microscope.

(c) 3-D transparent rendering of a sensorimotor cortical brain slice prepared from a 2-month-old VGAT-YFP-ChR2 (green) positive mouse (c) $\mathbf{c}_{1}$. L2 neuron (red) filled with alexa $594(0.2 \mathrm{mg} / \mathrm{ml}$; Invitrogen, A10438) (c2), ChR2-expressing cells (c3), and positions of the soma of ChR2expressing cell 3 in the red circle and stimulating laserspritzer $\left(\mathbf{c}_{4}\right)$ under differential interference contrast and/or fluorescence microscopy. Arrowheads indicate the somata of interneurons expressing YFP-ChR2 and numbers 1-8 indicate cells stimulated by laserspritzer.

(d) Laserspritzer stimulation $\left(8 \mathrm{~ms} ; 0.1 \mathrm{~mW} / \mathrm{mm}^{2}\right)$ at two of eight ChR2-expressing cells elicited IPSCs with short $(<6 \mathrm{~ms})$ and fixed latencies in postsynaptic L2 neuron. Note 10 consecutive recording traces shown in black, average sub- and supra-threshold responses shown in red, and laser stimulation indicated by blue bars. These animal experiments were approved by the University of Wyoming appropriate institutional animal care and use committee. 


\section{Figure 5. Single- and two-photon laser-based synaptic connection "search" technique.}

(a) Action potential thresholds for cortical L5 pyramidal neurons expressing either ChR2 $(\leq 20$ $\mu \mathrm{W}, n=1 ; 20-200 \mu \mathrm{W}, n=7 ; 200-2,000 \mu \mathrm{W}, n=11 ;>2 \mathrm{~mW}, n=4$ out of 23 neurons tested) or CheRiff ( $<20 \mu \mathrm{W}, n=33 ; 20-200 \mu \mathrm{W}, n=4$ out of 37 neurons tested) in respond to a $5 \mathrm{~ms} 473$ nm single-photon laser pulse.

(b) Left, schematic graph shows the photostimulation started with a $20 \mathrm{~ms} 920 \mathrm{~nm}$ two-photon spiral laser scanning followed by a $5 \mathrm{~ms} 473 \mathrm{~nm}$ single-photon laser pulse. Right, responses in a CheRiff-GFP expressing cortical L5 pyramidal neuron to a $5 \mathrm{~ms} 473 \mathrm{~nm}$ supra-threshold singlephoton laser pulse stimulation alone (cyan trace), a $20 \mathrm{~ms} 920 \mathrm{~nm}$ sub-threshold two-photon laser spiral scanning stimulation alone (red trace), a $5 \mathrm{~ms} 473 \mathrm{~nm}$ sub-threshold single-photon laser pulse stimulation alone (blue trace), and the combination of the sub-threshold two-photon laser spiral scanning and single-photon laser pulse stimulation (pink trace) under a 40x/0.8 NA Olympus objective lens.

(c) Top, responses of a CheRiff-GFP expressing cortical L5 pyramidal neuron to the suprathreshold single-photon laser pulse stimulation (cyan traces), and the combination of the subthreshold two-photon laser spiral scanning and single-photon laser pulse stimulation (pink traces) with the objective lens focusing point moving away from the soma. Bottom, plot of the average supra-threshold action potential responses of CheRiff-GFP expressing L5 pyramidal neurons against the distances between the laser focusing spot and soma of L5 pyramidal neurons $(n=21)$. Note the half-height spatial resolution of the combined single- and two-photon stimulation $(\sim 30$ $\mu \mathrm{m})$ is smaller than that of the single-photon stimulation alone $(\sim 60 \mu \mathrm{m})$. 
(d) Reconstruction of four L5 pyramidal neurons recorded simultaneously from an acute cortical slice superimposed on the transmitted light image captured during the recordings. The double colored dots indicate the putative synaptic contacts identified by light microscopy.

(e) The schematic drawing shows symbolically the synaptic connections.

(f) Upper, the combined single- and two-photon optogenetic stimulation of CheRiff-GFP expressing cortical L5 pyramidal neuron (green) evoked uEPSCs in one of L5 pyramidal neuron (orange), but not two others (blue and red). The monosynaptic connection was confirmed after CheRiff-GFP expressing neuron was patched and electrically stimulated in the whole-cell configuration. Note the slightly smaller amplitude and longer kinetics of average uEPSC evoked by the optogenetic stimulation compared to that evoked by the current injection in postsynaptic neuron due to the slight jittering of optogenetically evoked action potentials in presynaptic neuron. The majority of the unconnected axonal branches of the pyramidal neurons are not reconstructed for simplicity, and these animal experiments were approved by the University of Virginia appropriate institutional animal care and use committee. 
Figure 1

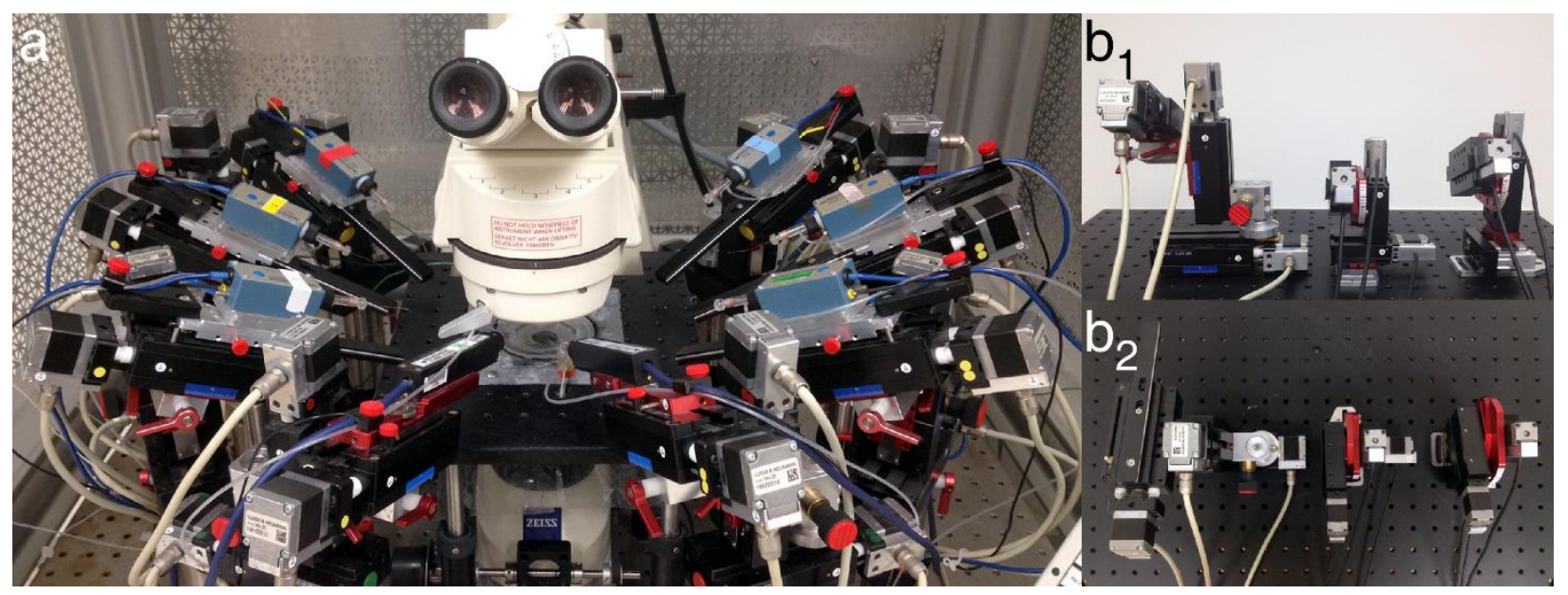


Figure 2

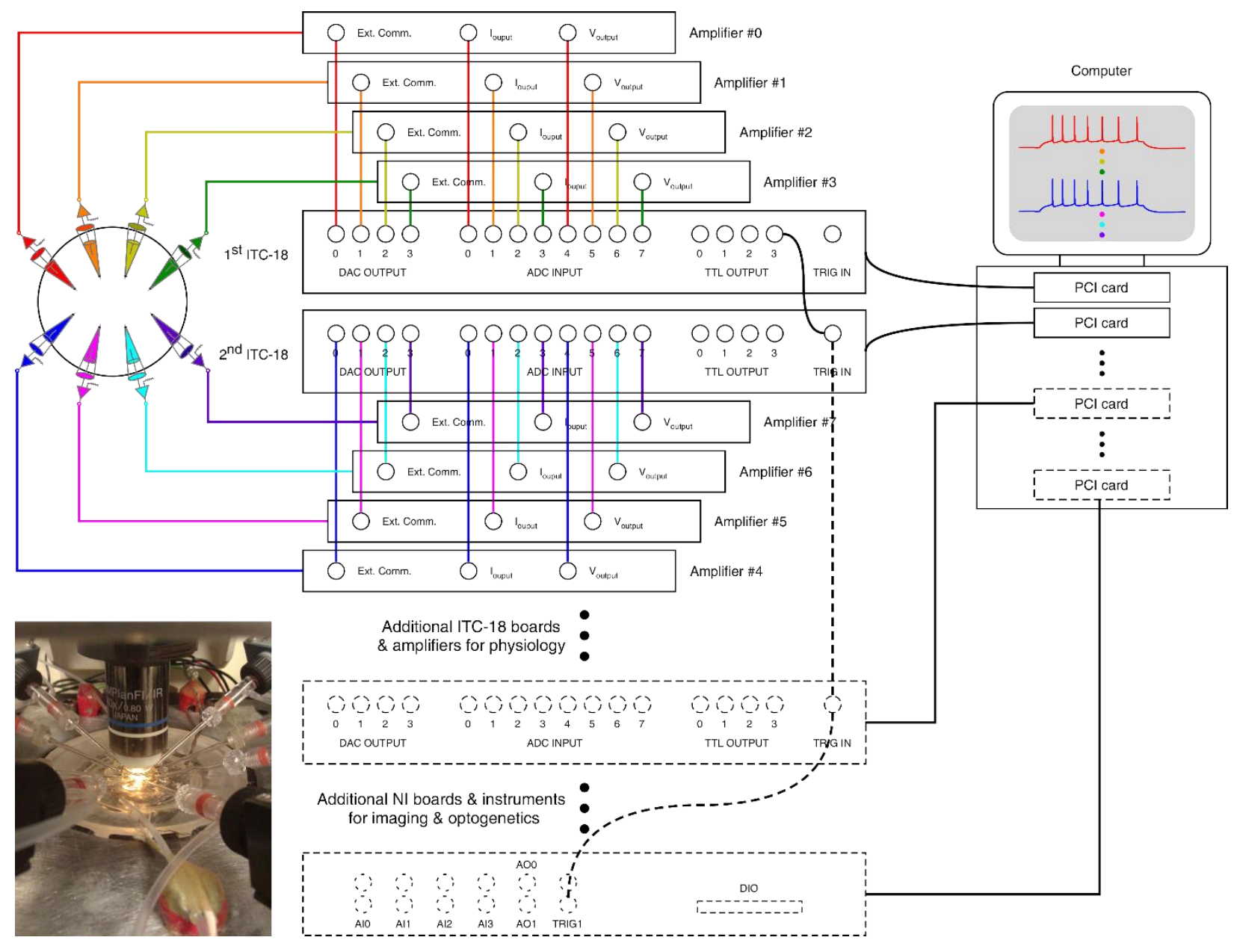


Figure 3
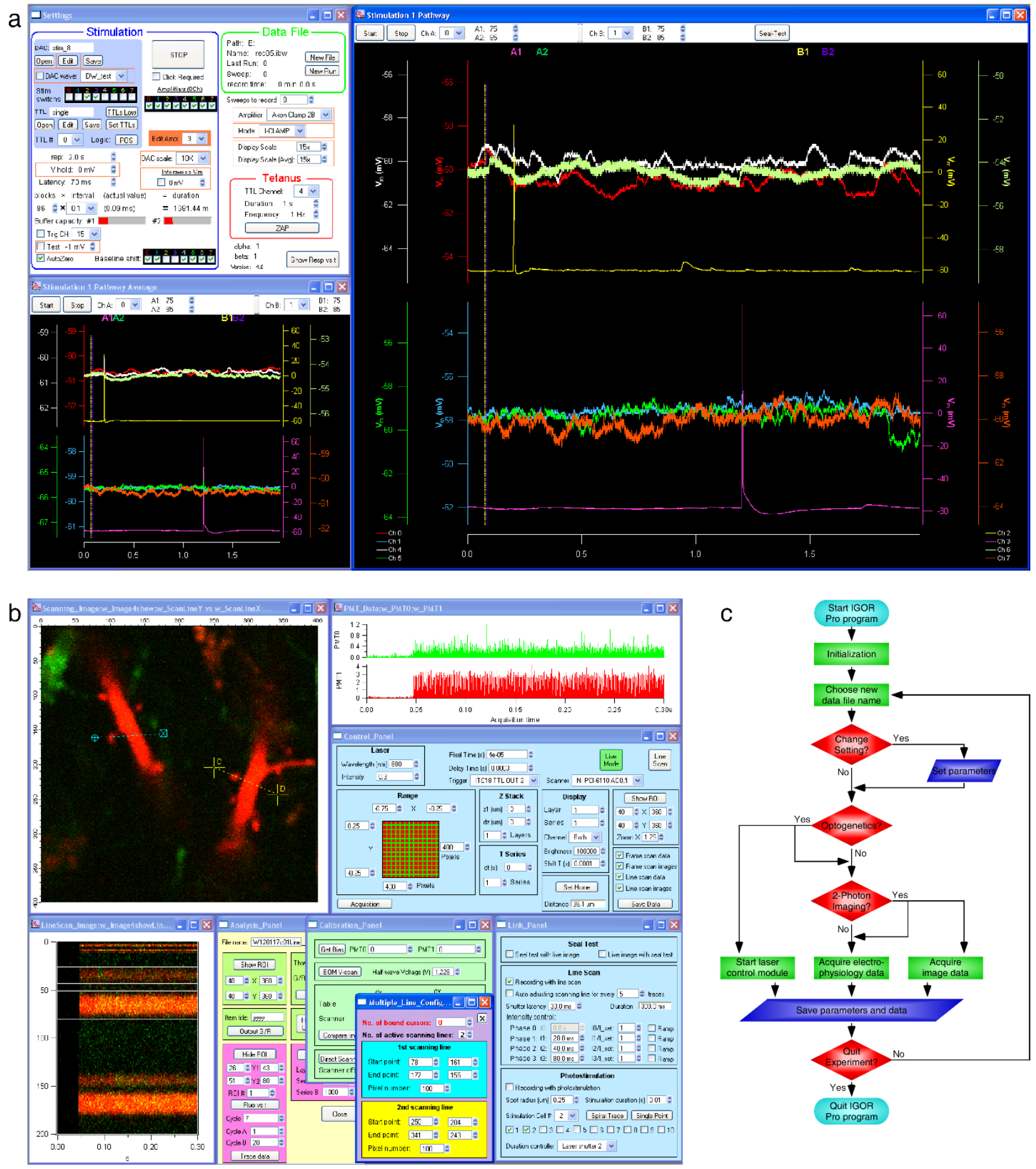
Figure 4

a

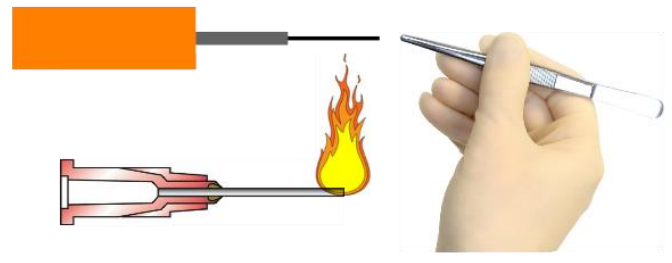

Preparation of laserspritzer

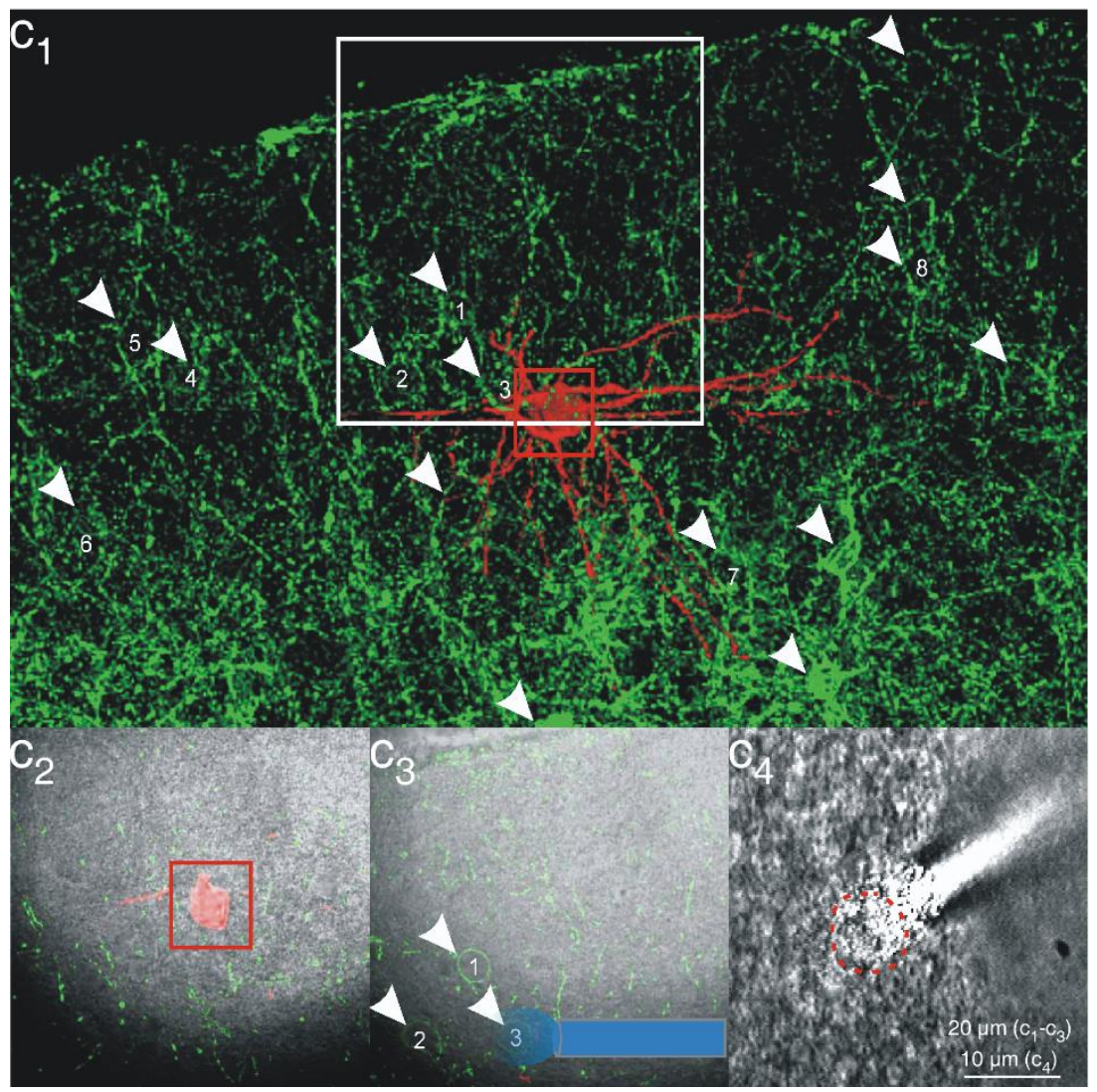

d cell

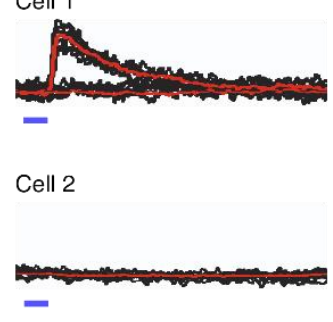

Cell 3

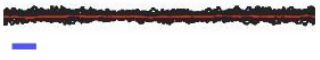

Cell 4

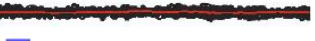

Cell 5

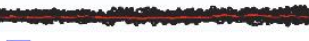

Cell 6

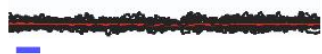

Cell 7

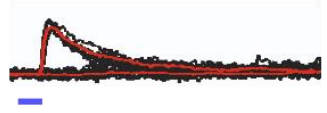

Cell 8

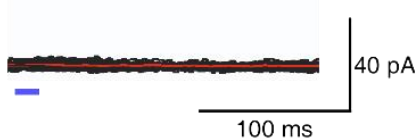


Figure 5

a

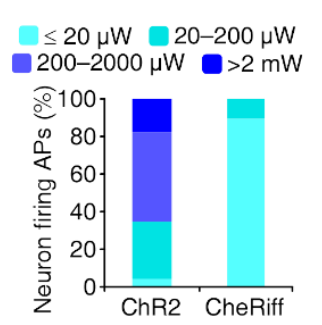

b

1- and 2-photon stimulation
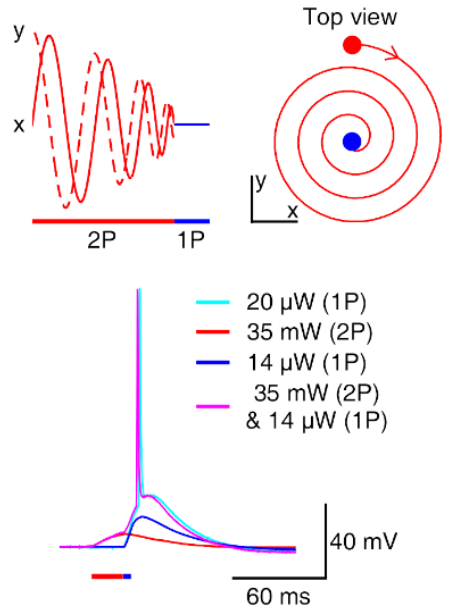

C
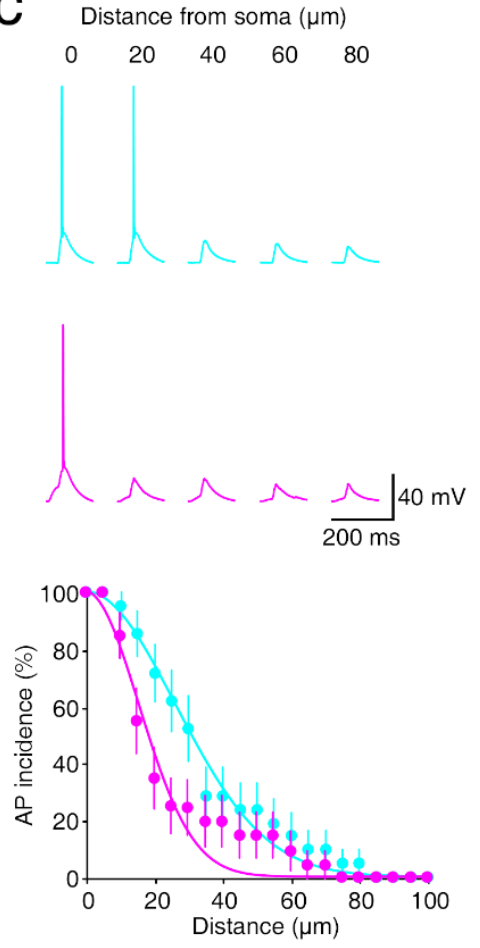

d

L1

L2/3

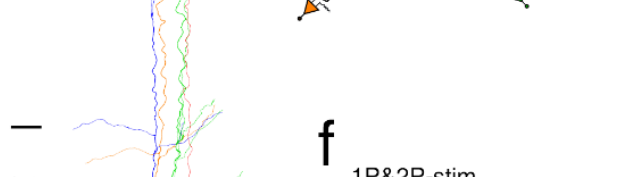

L4
-

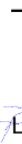

t5

$-$

L6

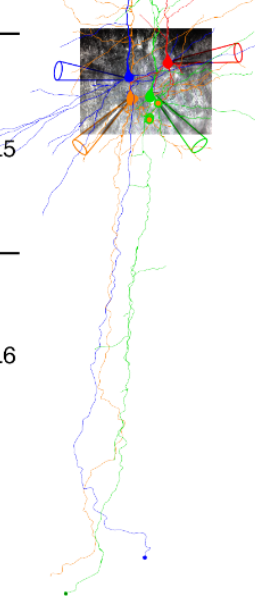

e

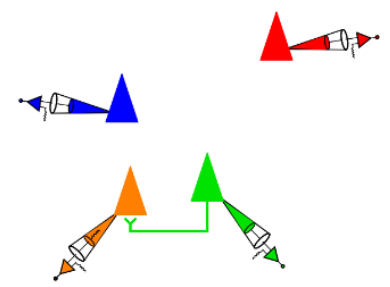

f

1P\&2P-stim

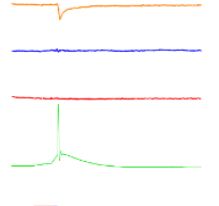

$-$

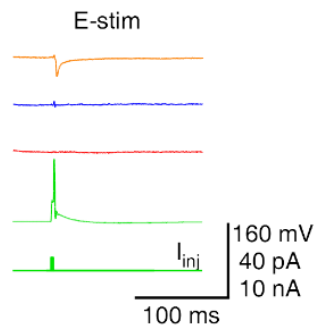




\section{References}

Brown, S. P., \& Hestrin, S. (2009a). Cell-type identity: a key to unlocking the function of neocortical circuits. Curr Opin Neurobiol, 19(4), 415-421.

Brown, S. P., \& Hestrin, S. (2009b). Intracortical circuits of pyramidal neurons reflect their longrange axonal targets. Nature, 457(7233), 1133-1136.

Buchanan, K. A., Blackman, A. V., Moreau, A. W., Elgar, D., Costa, R. P., Lalanne, T., . . . Sjostrom, P. J. (2012). Target-specific expression of presynaptic NMDA receptors in neocortical microcircuits. [Research Support, Non-U.S. Gov't]. Neuron, 75(3), 451-466. doi: 10.1016/j.neuron.2012.06.017

Chen, X., Leischner, U., Varga, Z., Jia, H., Deca, D., Rochefort, N. L., \& Konnerth, A. (2012). LOTOS-based two-photon calcium imaging of dendritic spines in vivo. [Research Support, Non-U.S. Gov't]. Nat Protoc, 7(10), 1818-1829. doi: 10.1038/nprot.2012.106

Constantinople, C. M., \& Bruno, R. M. (2013). Deep cortical layers are activated directly by thalamus. [Research Support, N.I.H., Extramural

Research Support, Non-U.S. Gov't

Research Support, U.S. Gov't, Non-P.H.S.]. Science, 340(6140), 1591-1594. doi: $10.1126 /$ science. 1236425

Couey, J. J., Witoelar, A., Zhang, S. J., Zheng, K., Ye, J., Dunn, B., . . Witter, M. P. (2013). Recurrent inhibitory circuitry as a mechanism for grid formation. [Research Support, NonU.S. Gov't]. Nat Neurosci, 16(3), 318-324. doi: 10.1038/nn.3310 
Davie, J. T., Kole, M. H., Letzkus, J. J., Rancz, E. A., Spruston, N., Stuart, G. J., \& Hausser, M. (2006). Dendritic patch-clamp recording. Nat Protoc, 1(3), 1235-1247.

Debanne, D., Boudkkazi, S., Campanac, E., Cudmore, R. H., Giraud, P., Fronzaroli-Molinieres, L., . . . Caillard, O. (2008). Paired-recordings from synaptically coupled cortical and hippocampal neurons in acute and cultured brain slices. [Comparative Study

Research Support, Non-U.S. Gov't]. Nat Protoc, 3(10), 1559-1568. doi: 10.1038/nprot.2008.147

Edwards, F. A., Konnerth, A., Sakmann, B., \& Takahashi, T. (1989). A thin slice preparation for patch clamp recordings from neurones of the mammalian central nervous system. Pflugers Arch, 414(5), 600-612.

Feldmeyer, D., Egger, V., Lubke, J., \& Sakmann, B. (1999). Reliable synaptic connections between pairs of excitatory layer 4 neurones within a single 'barrel' of developing rat somatosensory cortex. J Physiol, 521 Pt 1, 169-190.

Feng, L., Kwon, O., Lee, B., Oh, W. C., \& Kim, J. (2014). Using mammalian GFP reconstitution across synaptic partners (mGRASP) to map synaptic connectivity in the mouse brain. [Research Support, N.I.H., Extramural

Research Support, Non-U.S. Gov't]. Nat Protocols, 9(10), 2425-2437. doi: 10.1038/nprot.2014.166

Flytzanis, N. C., Bedbrook, C. N., Chiu, H., Engqvist, M. K., Xiao, C., Chan, K. Y., .. Gradinaru, V. (2014). Archaerhodopsin variants with enhanced voltage-sensitive fluorescence in mammalian and Caenorhabditis elegans neurons. Nat Commun, 5, 4894. doi: $10.1038 /$ ncomms5894 
Geiger, J. R., Bischofberger, J., Vida, I., Frobe, U., Pfitzinger, S., Weber, H. J., . . Jonas, P. (2002). Patch-clamp recording in brain slices with improved slicer technology. Pflugers Arch, 443(3), 491-501.

Hochbaum, D. R., Zhao, Y., Farhi, S. L., Klapoetke, N., Werley, C. A., Kapoor, V., . . Cohen, A. E. (2014). All-optical electrophysiology in mammalian neurons using engineered microbial rhodopsins. [Research Support, N.I.H., Extramural

Research Support, Non-U.S. Gov't

Research Support, U.S. Gov't, Non-P.H.S.]. Nat Methods, 11(8), 825-833. doi: 10.1038/nmeth.3000

Jiang, X., Wang, G., Lee, A. J., Stornetta, R. L., \& Zhu, J. J. (2013). The organization of two new cortical interneuronal circuits. Nat Neurosci, 16(2), 210-218. doi: 10.1038/nn.3305

Kim, J., Zhao, T., Petralia, R. S., Yu, Y., Peng, H., Myers, E., \& Magee, J. C. (2012). mGRASP enables mapping mammalian synaptic connectivity with light microscopy. [Research Support, N.I.H., Extramural

Research Support, Non-U.S. Gov't]. Nat Methods, 9(1), 96-102. doi: 10.1038/nmeth.1784

Kitamura, K., Judkewitz, B., Kano, M., Denk, W., \& Hausser, M. (2008). Targeted patch-clamp recordings and single-cell electroporation of unlabeled neurons in vivo. Nat Methods, 5(1), 61-67.

Komai, S., Denk, W., Osten, P., Brecht, M., \& Margrie, T. W. (2006). Two-photon targeted patching (TPTP) in vivo. [Research Support, Non-U.S. Gov't]. Nat Protoc, 1(2), 647-652. doi: $10.1038 /$ nprot.2006.100 
Kozloski, J., Hamzei-Sichani, F., \& Yuste, R. (2001). Stereotyped position of local synaptic targets in neocortex. Science, 293(5531), 868-872. doi: 10.1126/science.293.5531.868

Larkum, M. E., \& Zhu, J. J. (2002). Signaling of layer 1 and whisker-evoked $\mathrm{Ca}^{2+}$ and $\mathrm{Na}^{+}$action potentials in distal and terminal dendrites of rat neocortical pyramidal neurons in vitro and in vivo. J Neurosci, 22(16), 6991-7005.

Larkum, M. E., Zhu, J. J., \& Sakmann, B. (1999). A new cellular mechanism for coupling inputs arriving at different cortical layers. Nature, 398(6725), 338-341.

Le Be, J. V., \& Markram, H. (2006). Spontaneous and evoked synaptic rewiring in the neonatal neocortex. Proc Natl Acad Sci U S A, 103(35), 13214-13219.

Lee, A. J., Wang, G., Jiang, X., Johnson, S. M., Hoang, E. T., Lante, F., . . Julius Zhu, J. (2014). Canonical organization of layer 1 neuron-led cortical inhibitory and disinhibitory interneuronal circuits. Cereb Cortex. doi: 10.1093/cercor/bhu020

Lee, A. K., Epsztein, J., \& Brecht, M. (2009). Head-anchored whole-cell recordings in freely moving rats. [Research Support, Non-U.S. Gov't]. Nat Protoc, 4(3), 385-392. doi: 10.1038/nprot.2009.5

Lefort, S., Tomm, C., Floyd Sarria, J. C., \& Petersen, C. C. (2009). The excitatory neuronal network of the C2 barrel column in mouse primary somatosensory cortex. Neuron, 61(2), 301-316.

Luo, L., Callaway, E. M., \& Svoboda, K. (2008). Genetic dissection of neural circuits. Neuron, 57(5), 634-660. 
Markram, H., Lubke, J., Frotscher, M., Roth, A., \& Sakmann, B. (1997). Physiology and anatomy of synaptic connections between thick tufted pyramidal neurones in the developing rat neocortex. J Physiol (Lond), 500(Pt 2), 409-440.

Marx, M., Gunter, R. H., Hucko, W., Radnikow, G., \& Feldmeyer, D. (2012). Improved biocytin labeling and neuronal 3D reconstruction. [Research Support, Non-U.S. Gov't]. Nat Protoc, 7(2), 394-407. doi: 10.1038/nprot.2011.449

Murayama, M., \& Larkum, M. E. (2009). In vivo dendritic calcium imaging with a fiberoptic periscope system. Nat Protoc, 4(10), 1551-1559.

Osakada, F., \& Callaway, E. M. (2013). Design and generation of recombinant rabies virus vectors. [Research Support, N.I.H., Extramural

Research Support, Non-U.S. Gov't]. Nat Protoc, 8(8), 1583-1601. doi: 10.1038/nprot.2013.094

Perin, R., Berger, T. K., \& Markram, H. (2011). A synaptic organizing principle for cortical neuronal groups. Proc Natl Acad Sci U S A, 108(13), 5419-5424.

Petreanu, L., Huber, D., Sobczyk, A., \& Svoboda, K. (2007). Channelrhodopsin-2-assisted circuit mapping of long-range callosal projections. Nat Neurosci, 10(5), 663-668.

Pologruto, T. A., Sabatini, B. L., \& Svoboda, K. (2003). ScanImage: flexible software for operating laser scanning microscopes. [Evaluation Studies

Research Support, U.S. Gov't, P.H.S.]. Biomed Eng Online, 2, 13. doi: 10.1186/1475-925X-2-13

Reyes, A., \& Sakmann, B. (1999). Developmental switch in the short-term modification of unitary EPSPs evoked in layer $2 / 3$ and layer 5 pyramidal neurons of rat neocortex. $J$ Neurosci, 19(10), 3827-3835. 
Scanziani, M., \& Hausser, M. (2009). Electrophysiology in the age of light. Nature, 461(7266), 930-939.

Schiller, J., Schiller, Y., Stuart, G., \& Sakmann, B. (1997). Calcium action potentials restricted to distal apical dendrites of rat neocortical pyramidal neurons. J Physiol (Lond), 505(Pt 3), 605-616.

Spruston, N., Schiller, Y., Stuart, G., \& Sakmann, B. (1995). Activity-dependent action potential invasion and calcium influx into hippocampal CA1 dendrites. Science, 268(5208), 297300.

Stern, E. A., Maravall, M., \& Svoboda, K. (2001). Rapid development and plasticity of layer 2/3 maps in rat barrel cortex in vivo. Neuron, 31(2), 305-315.

Stuart, G. J., \& Sakmann, B. (1994). Active propagation of somatic action potentials into neocortical pyramidal cell dendrites. Nature, 367(6458), 69-72.

Sun, Q. Q., Wang, X., \& Yang, W. (2014). Laserspritzer: a simple method for optogenetic investigation with subcellular resolutions. PLoS One, 9(7), e101600. doi: 10.1371/journal.pone. 0101600

Suter, B. A., O'Connor, T., Iyer, V., Petreanu, L. T., Hooks, B. M., Kiritani, T., . . Shepherd, G. M. (2010). Ephus: multipurpose data acquisition software for neuroscience experiments. Front Neural Circuits, 4, 100. doi: 10.3389/fncir.2010.00100

Tang, Q., Brecht, M., \& Burgalossi, A. (2014). Juxtacellular recording and morphological identification of single neurons in freely moving rats. Nat Protoc, 9(10), 2369-2381. doi: 10.1038/nprot.2014.161 
Tomer, R., Ye, L., Hsueh, B., \& Deisseroth, K. (2014). Advanced CLARITY for rapid and highresolution imaging of intact tissues. [Research Support, N.I.H., Extramural

Research Support, Non-U.S. Gov't

Research Support, U.S. Gov't, Non-P.H.S.]. Nat Protoc, 9(7), 1682-1697. doi: 10.1038/nprot.2014.123

Waters, J., Larkum, M., Sakmann, B., \& Helmchen, F. (2003). Supralinear Ca2+ influx into dendritic tufts of layer $2 / 3$ neocortical pyramidal neurons in vitro and in vivo. $J$ Neurosci, 23(24), 8558-8567.

Wickersham, I. R., Lyon, D. C., Barnard, R. J., Mori, T., Finke, S., Conzelmann, K. K., . . . Callaway, E. M. (2007). Monosynaptic restriction of transsynaptic tracing from single, genetically targeted neurons. Neuron, 53(5), 639-647.

Wu, G. K., Tao, H. W., \& Zhang, L. I. (2011). From elementary synaptic circuits to information processing in primary auditory cortex. [Research Support, N.I.H., Extramural

Research Support, Non-U.S. Gov't]. Neurosci Biobehav Rev, 35(10), 2094-2104.

Xue, M., Atallah, B. V., \& Scanziani, M. (2014). Equalizing excitation-inhibition ratios across visual cortical neurons. Nature, 511(7511), 596-600. doi: 10.1038/nature13321

Yu, Y. C., Bultje, R. S., Wang, X., \& Shi, S. H. (2009). Specific synapses develop preferentially among sister excitatory neurons in the neocortex. Nature, 458(7237), 501-504.

Zhao, S., Ting, J. T., Atallah, H. E., Qiu, L., Tan, J., Gloss, B., . . Feng, G. (2011). Cell typespecific channelrhodopsin-2 transgenic mice for optogenetic dissection of neural circuitry function. [Research Support, American Recovery and Reinvestment Act 
Research Support, N.I.H., Extramural

Research Support, Non-U.S. Gov't]. Nat Methods, 8(9), 745-752.

Zhu, J. J. (2000). Maturation of layer 5 neocortical pyramidal neurons: amplifying salient layer 1 and layer 4 inputs by $\mathrm{Ca}^{2+}$ action potentials in adult rat tuft dendrites. $J$ Physiol (Lond), 526(Pt 3), 571-587.

Zhu, J. J., \& Connors, B. W. (1999). Intrinsic firing patterns and whisker-evoked synaptic responses of neurons in the rat barrel cortex. J Neurophysiol, 81(3), 1171-1183.

Zhu, Y., Stornetta, R. L., \& Zhu, J. J. (2004). Chandelier cells control excessive cortical excitation: characteristics of whisker-evoked synaptic responses of layer $2 / 3$ nonpyramidal and pyramidal neurons. J Neurosci, 24(22), 5101-5108.

Zhu, Y., \& Zhu, J. J. (2004). Rapid arrival and integration of ascending sensory information in layer 1 nonpyramidal neurons and tuft dendrites of layer 5 pyramidal neurons of the neocortex. J Neurosci, 24(6), 1272-1279. 


\section{CHAPTER 3}

\section{Analysis of transsynaptic attentional neuronal circuits with multiple ( $\geq 4)$ whole-cell recordings}

Daniel R. Wyskiel ${ }^{1,2}$, Trevor C. Larry ${ }^{1,3}$, Xiaolong Jiang ${ }^{1}$, Guangfu Wang ${ }^{1}$, J. Julius Zhu ${ }^{1}$

Departments of Pharmacology ${ }^{1}$, Neuroscience Graduate Program ${ }^{2}$ and Neuroscience Undergraduate Program ${ }^{3}$, University of Virginia School of Medicine, Charlottesville, VA 22908, USA, 
Deciphering interneuronal circuitry is essential to understanding brain functions yet remains a daunting task in neurobiology. To facilitate the dissection of complex cortical neuronal circuits, a process requiring analysis of synaptic interconnections and identification of cell types of interconnected neurons, I helped developed a simultaneous quadruple-octuple whole-cell recordings technique that allows physiological analysis of synaptic interconnection among up to eight neurons and anatomical identification of the majority of recorded neurons. Using this method, the Julius Zhu have recently revealed two transsynaptic disinhibitory and inhibitory circuits connecting layer 1-3 interneurons with pyramidal neurons in both supragranular and infragranular cortical layers of the rat neocortex. Here, I outline the technique that permits decoding the complex cortical interneuronal circuits involved in controlling salience detection.

Key words: Multiple whole-cell recordings, interneurons, circuits, coincidence detection, salience. 


\section{BACKGROUND}

The cerebral cortex is a multilayered structure responsible for many higher-order functions, including those involved in attention (Gilbert and Wiesel 1983; Mountcastle 1997; Kastner and Ungerleider 2000; Douglas and Martin 2007). Layer 1 of the cerebral cortex (L1) is strategically positioned to regulate the pathways involved in attention as it receives projections from both higher-order thalamic nuclei and higher-order cortical areas, two regions known to be critical for the selection of salient information (Robinson and Petersen 1992; Tomita et al. 1999; PascualLeone and Walsh 2001; Gilbert and Sigman 2007; van Boxtel et al. 2010; Baluch and Itti 2011; Purushothaman et al. 2012; van Gaal and Lamme 2012; Larkum 2013). These inputs generate direct excitatory postsynaptic potentials in L1 (Zhu and Zhu 2004; Zhu 2009; Jiang et al. 2013), and the excitation is selectively and dramatically enhanced during attentional tasks (Kuhn et al. 2008; Cauller and Kulics 1991; Letzkus et al. 2011). Moreover, L1 receives dense innervations from neuromodulatory systems that may robustly modulate neuronal activity in L1 (Christophe et al. 2002; Yuen and Yan 2009; Letzkus et al. 2011).

L1 is composed of almost entirely GABAergic interneurons that fall into two general groups (Chu et al. 2003; Zhu and Zhu 2004; Wozny and Williams 2011; Kubota et al. 2011; Cruikshank et al. 2012; Jiang et al. 2013; Ma et al. 2013; Muralidhar et al. 2013; Lee et al. 2014). One group of L1 interneurons has heterogeneous dendritic morphology and axons projecting into deeper layers of the cortex, whereas the other group of L1 interneurons is multipolar, aspiny interneurons with highly ramified axons projecting horizontally within L1. These two groups of interneurons generally fire adapting non-late-spiking and non-adapting late-spiking action potentials, respectively. However, exceptions to this cell morphology-firing pattern correlation have been 
reported. However, exceptions to this cell morphology-firing pattern correlation have been reported (Kubota et al. 2011), and confirmed with a much larger L1 interneuron sample (Jiang et al. 2013). On the other hand, these two groups of interneurons can be unambiguously defined as single-bouquet cells (SBCs) and elongated neurogliaform cells (ENGCs) based on their visually distinguishable axonal arborization patterns (Jiang et al. 2013), following the recently proposed nomenclature (Ascoli et al. 2008).

Pyramidal neurons, the primary excitatory neurons in the cortex, contain long apical dendrites which largely contribute to the synaptic integration that ultimately forms the basis of the brain's neuronal computational power (Reyes 2001; Sjostrom et al. 2008; Spruston 2008; Larkum 2013). L2/3 and L5 pyramidal neurons, the major cortical output neurons, send apical dendrites that terminate in L1, where they are innervated by attention-related thalamic and cortical inputs (Rockland and Pandya 1979; DeFelipe and Farinas 1992; Johnson and Burkhalter 1997; Cauller et al. 1998; Petreanu et al. 2009). These modulatory synaptic inputs to distal apical dendrites of L2/3 and L5 pyramidal neurons can induce dendritic action potentials if of sufficient amplitude (Schiller et al. 1997; Zhu and Connors 1999; Zhu 2000; Larkum and Zhu 2002). Moreover, the induction of dendritic action potentials can be greatly facilitated by the back-propagation of action potentials elicited by concurrent L4 sensory inputs (Zhu and Connors 1999; Larkum and Zhu 2002; Antic 2003), resulting in dendritic complex spikes and bursts of somatic/axonal action potentials (Larkum et al. 1999; Larkum and Zhu 2002; Waters et al. 2003; Larkum et al. 2009; Xu et al. 2012). Because the dendritic complex spikes and bursts of somatic/axonal action potentials secure further processing of the concurrent synaptic signals (Lisman 1997; Sjostrom et al. 2008), they act as a coincidence detection mechanism for salient synaptic inputs. Therefore, interneurons from 
L1 may regulate initiation of dendritic complex spikes in L2/3 and L5 pyramidal neurons and thereby effectively control the coincidence detection or salience selection mechanisms.

The functional significance of L1 neurons and how they are integrated into the cortical circuit remain poorly understood. Specifically, the morphological differences between SBCs and ENGCs suggest different regulatory functions. However, due to the extensive, intricate synaptic organization between the vastly diverse interneurons and pyramidal neurons, deciphering complex neuronal circuits, such as those L1 interneuron-involved salience selection circuits, has been a daunting task. To combat this challenge, I helped developed a method that allows for stable wholecell recordings from up to eight neurons simultaneously and leads to morphological recovery and subsequent cell type identification of more than $85 \%$ of recorded interneurons and more than $99 \%$ of pyramidal neurons (Jiang et al. 2013).

Using the simultaneous quadruple-octuple whole-cell recordings technique I helped optomize (Fig 1), the Julius Zhu lab investigated L1 interneurons and their connections between L2/3 and L5 neurons (Jiang et al. 2013; Lee et al. 2014). Our analysis reveals that SBCs predominantly make unidirectional inhibitory connections $(\mathrm{SBC} \rightarrow$ ) with L2/3 interneurons, whereas ENGCs frequently form reciprocal inhibitory and electric connections (ENGC $\leftrightarrow$ ) with L2/3 interneurons. Axon arborization analysis identifies seven general interneuron subtypes in L2/3, including Martinotti cells (MaCs), neurogliaform cells (NGCs), bitufted cells (BTCs), bipolar cells (BPCs), basket cells (BaCs), double-bouquet cells (DBCs), and chandelier cells (ChCs) in L2/3. SBCs innervate all seven L2/3 interneuron subtypes. By contrast, ENGCs preferentially innervate three of these 
subtypes: MaCs, NGCs and BTCs. Simultaneous recordings from L1, L2/3 and L5 neurons show that $\mathrm{SBC} \rightarrow \mathrm{L} 2 / 3$ interneuronal circuits primarily disinhibit L2/3 and L5 pyramidal neurons in the same columns. Conversely, ENGC $\leftrightarrow \mathrm{L} 2 / 3$ interneuronal circuits directly inhibit L2/3 and L5 pyramidal neurons in the same and/or neighboring columns. These results support the notion that SBCs and ENGCs form distinct interneuronal circuits with both L2/3 interneurons and L2-5 pyramidal neurons such that $\mathrm{SBC} \rightarrow \mathrm{L} 2 / 3$ interneuronal circuits disinhibit whereas $\mathrm{ENGC} \leftrightarrow \mathrm{L} 2 / 3$ interneuronal circuits inhibit L2/3 and L5 pyramidal neurons. Furthermore, SBC-led interneuronal circuits disinhibit primarily the dendritic-somato-axonal axis of a small number of L2/3 and L5 pyramidal neurons in the same columns, whereas ENGC-led interneuronal circuits primarily inhibit the distal apical dendrite of much more of these cells in multiple columns.

The contrasting SBC- and ENGC-led interneuronal circuits with L2/3 and L5 pyramidal neurons suggest different functional roles for these circuits. Indeed, action potentials elicited in SBCs inhibit L2/3 interneurons, and relieve the suppression of complex dendritic spiking and somatic bursting in L5 pyramidal neurons. Conversely, action potentials initiated in ENGCs recruit and synchronize the activity in L2/3 interneurons, and potentiate the suppression of complex dendritic spiking and somatic bursting in L5 pyramidal neurons. In intact brains, paired recordings from SBCs and L5 pyramidal neurons show that spontaneous or whisker-evoked synaptic events can trigger action potentials in SBCs that enhance dendritic complex spiking in L5 pyramidal neurons. Conversely, paired recordings from ENGCs and L5 pyramidal neurons show that spontaneous or whisker-evoked synaptic events can trigger action potentials in ENGCs that suppress dendritic complex spiking in L5 pyramidal neurons. Altogether, these results consistently testify to the coexistence of two distinct L1-interneuron-led attention-related interneuronal circuits: $\mathrm{SBC} \rightarrow \mathrm{L} 2 / 3$ 
interneuron $\rightarrow$ L2-5 pyramidal neuronal circuits disinhibit the coincidence detection mechanism, whereas $\mathrm{ENGC} \leftrightarrow \mathrm{L} 2 / 3$ interneuron $\rightarrow \mathrm{L} 2-5$ pyramidal neuronal circuits inhibit the coincidence detection mechanism. These two distinct interneuronal circuits can transform L1 inputs into complementary "filters" by differentially regulating the output of L2/3 and L5 pyramidal neurons. Thus, the two interneuronal circuits seem to work synergistically with the dendritic coincidence detection mechanism in pyramidal neurons to filter out "noise" in the incoming information to achieve effective salience selection. 


\section{MATERIALS}

To ensure examination of mature and stabilized cortical inhibitory neurons and circuits (Huang et al. 2007; Batista-Brito and Fishell 2009), rodents postnatal 20 days or older should be used. When preparing acute brain slices, anesthetics, such as sodium pentobarbital, are required. Immediately upon extraction of the brain, an artificial cerebrospinal fluid (ACSF), saturated with carbogen $\left(95 \% \mathrm{O}_{2} / 5 \% \mathrm{CO}_{2}\right)$, will be needed. ACSF is prepared with the following ingredients: $\mathrm{NaCl}, \mathrm{KCl}$, $\mathrm{NaH}_{2} \mathrm{PO}_{4}, \mathrm{NaHCO}_{3}, \mathrm{MgCl}_{2}$, dextrose, and $\mathrm{CaCl}_{2}$. This solution should be cold $\left(0-4{ }^{\circ} \mathrm{C}\right)$ for the extraction and slicing procedure. A microslicer with minimal vibrations in the vertical axis is preferable to obtain healthy acute cortical slices (Geiger et al. 2002). After slices are obtained, a beaker containing ACSF saturated with carbogen and heated to $37.0 \pm 0.5^{\circ} \mathrm{C}$, will be needed to incubate the slices.

Electrophysiological recordings require two internal solutions (one for current clamp and one for voltage clamp) containing different combinations of the following components: cesium methanesulfonate, $\mathrm{HEPES}, \mathrm{MgCl}_{2}, \mathrm{Na}_{2} \mathrm{ATP}, \mathrm{Na}_{3} \mathrm{GTP}$, sodium phosphocreatine, EGTA, spermine, biocytin, potassium gluconate, $\mathrm{KCl}$ and $\mathrm{MgATP}$.

Eight amplifiers are needed to record from up to eight neurons simultaneously. I used Axopatch 200B and/or Axoclamp 2A/B amplifiers (Molecular Devices, Sunnyvale, CA), which are ideal for current and voltage recordings, respectively. Because no single commercially available interface board is able to supply enough $\mathrm{A} / \mathrm{D}$ and $\mathrm{D} / \mathrm{A}$ channels for eight amplifiers, at least two interface boards are needed. In our setup, two ITC-18 interface boards (HEKA Instruments Inc, Bellmore, 
$\mathrm{NY}$ ) are used to achieve simultaneous A/D and D/A conversions of current, voltage, command and triggering signal for up to eight amplifiers. In addition, custom-written Igor-based software programs are used to synchronize two ITC-18 boards, operate eight Axon amplifiers, and perform online and offline data analysis. Stable long-lasting recordings are crucial to fill sufficient biocytin to recover the complex axonal arborization of recorded interneurons. In our setup, L\&N Mini motorized manipulators (Luigs \& Neumann Feinmechanik and Elektrotechnik, Ratingen, Germany) are used because of their excellent stability and compactness. Furthermore, their headstage rails are specially designed to minimize the loss of other recordings when the exchange of patch pipettes becomes necessary.

Immunohistochemistry procedures are carried out with several toxic reagents. The slices are first fixed in a saline solution containing acrolein, paraformaldehyde, and phosphate (used to buffer the solution). After 24 hours, the slices are processed with avidin-biotin-peroxidase and some require additional fixation with $\mathrm{OsO}_{4}$ and uranyl acetate. A computerized reconstruction system (Neurolucida, Williston, VT) is needed to reconstruct recorded neurons and analyze their morphological properties. For electron microscopy, an ultramicrotome is required for preparing ultrathin tissue sections, and an electron microscope, such as a JEOL-1230 transmission electron microscope (Japan Electron Optic, Tokyo, Japan), is required for visualization of synapses. 


\section{METHODS}

All procedures for animal surgery and maintenance are performed following experimental protocols in accordance with U.S. National and Institutional Guidelines for the Care and Use of Laboratory Animals.

\subsection{In Vitro Multiple Whole-Cell Patch-Clamp Recordings}

\subsubsection{Preparing the Artificial Cerebrospinal Fluid (ACSF)}

Proper preparation of ACSF is essential to the success of whole-cell patch-clamp recordings. To produce 10 liters of ACSF solution, add $69.54 \mathrm{~g} \mathrm{NaCl}(119 \mathrm{mM}), 1.86 \mathrm{~g} \mathrm{KCl}(2.5 \mathrm{mM}), 21.84 \mathrm{~g}$ $\mathrm{NaHCO}_{3}(26 \mathrm{mM})$, and $1.2 \mathrm{~g} \mathrm{Na}_{2} \mathrm{PO}_{4}(1 \mathrm{mM})$ in 9.5 liters of double distilled water. The solution is stirred during the addition of these reagents, and then filled to the 10 liter mark with additional double distilled water. This solution is stable and can be stored for months until it is needed, at which time $4.5 \mathrm{~g}$ of dextrose, $1 \mathrm{ml}$ of a $1 \mathrm{M} \mathrm{MgCl}_{2}$ solution and $2 \mathrm{ml}$ of a $1 \mathrm{M} \mathrm{CaCl}_{2}$ solution are added to one liter of the solution.

\subsubsection{Acute Cortical Brain Slice Preparation}

As mentioned above, male and female rodents $\geq 20$ days old are to be used for the preparation of acute cortical slices. The animals are anesthetized by an intraperitoneal injection of sodium pentobarbital at a ratio of $90 \mathrm{mg} / \mathrm{kg}$ of body weight. Once deep anesthesia has been established, the animals are decapitated, the skin and skull cut open, and then pulled back with curved, blunt forceps. Icy ACSF $\left(0-4{ }^{\circ} \mathrm{C}\right)$ is immediately poured onto the brain to limit cell death. The brain 
region of interest is extracted and put directly into a beaker of oxygenated and iced ACSF to cool. Apply a thin layer of glue to the specimen plate. After a brief placement of the brain block on a piece of filter paper to remove excess ACSF, it can be glued to the specimen plate of the microslicer. Icy ACSF is immediately poured to cover the tissue. Parasagittal slices $350 \mu \mathrm{m}$ thick are cut from the tissue block at an angle $\left(<\sim 4^{\circ}\right)$ closely parallel to apical dendrites of L5 pyramidal neurons, which should retain the majority of distal ascending and descending axonal trees of L13 interneurons that project into L1 and L5-6. The brain slices are gently transferred to a beaker of oxygenated ACSF kept in a water bath at $37.0 \pm 0.5^{\circ} \mathrm{C}$ for $\sim 30$ minutes before recordings.

\subsubsection{Whole-Cell Patch-Clamp Recording}

For whole-cell patch-clamp recordings, the determination of patch-pipette solutions depends upon the desired measurement: current or voltage. For current recordings, fill the pipette with $135 \mathrm{mM}$ cesium methanesulfonate, $10 \mathrm{mM}$ HEPES, $2.5 \mathrm{mM} \mathrm{MgCl}_{2}, 4 \mathrm{mM} \mathrm{Na} 2 \mathrm{ATP}, 0.4 \mathrm{mM} \mathrm{Na} 3 \mathrm{GTP}, 10$ $\mathrm{mM}$ sodium phosphocreatine, $0.6 \mathrm{mM}$ EGTA, $0.1 \mathrm{mM}$ spermine, and $0.5 \%$ biocytin. For voltage recordings, $120 \mathrm{mM}$ potassium gluconate, $10 \mathrm{mM}$ HEPES, $4 \mathrm{mM} \mathrm{KCl,} 4 \mathrm{mM} \mathrm{MgATP}, 0.3 \mathrm{mM}$ $\mathrm{Na}_{3} \mathrm{GTP}, 10 \mathrm{mM}$ sodium phosphocreatine and $0.5 \%$ biocytin are used in the recording pipette. The addition of biocytin to these solutions allows for diffusion of the compound from the pipette into neurons during the recording so that histological staining and proceeding morphological analysis can be achieved thereafter. The resistance of the pipette is between 3-7 M $\Omega$.

To perform electrophysiological recordings, transfer a cortical slice to the recording. The recording bath should be continuously prefused with ACSF, saturated with carbogen, and held at 
$34.0 \pm 0.5{ }^{\circ} \mathrm{C}$. The cortical slice is held in place on the chamber using a platinum ring covered with a grid of nylon strings, similar to one described by Edwards et al. in 1989 (Edwards et al. 1989). Ideally, the entire apical dendrite of a pyramidal neuron can be visually tracked with little adjustment of the focus. It is good practice to identify and select healthy neurons to be recorded before placing the electrodes slightly above the tissue. Paramount to formation of tight G $\Omega$ seals is the maintenance of clean pipette tips. As the pipettes are lowered through the tissue, positive pressure should be applied to the pipettes so as to push the tissue away from the tip of the pipette, which also helps to clean off the membrane debris around the neurons as the electrodes approach them. Tight $\mathrm{G} \Omega$ seal recordings can be achieved following the previously established procedure (Edwards et al. 1989). In order to establish multiple recordings from eight neurons simultaneously, many different exercises should be adhered to. First and foremost, it is imperative that the rig be properly assembled, maintained and fully functional (a detailed description on the setup needed for in vitro recordings can be found in Molecular Devices Axon Guide and Davie et al., 2006). Furthermore, continuous practice is needed to achieve the skills necessary to complete it in a timely manner.

\subsubsection{Identification of Inhibitory Synaptic Events}

Although it is often most helpful to determine all the cells you wish to patch prior to starting, in some cases this may not be preferred. If a particular cell type is desired, such as a fast-spiking interneuron, it may be beneficial to examine the electrophysiological properties before selecting the next cells. It should be noted that although somatic morphology and membrane and firing characteristics help in identifying inhibitory interneurons, there is no certainty that the cell is inhibitory unless it inhibits a postsynaptic neuron. Another technique one might also employ is 
different "searching" techniques to find a connected neuron before patching as described by Feldmeyer. Regardless of the above strategies, once all the cells have been selected it is best to immerse each electrode into the bath, zero any offsets, establish the desired positive pressure, and lower each one to just above the slice. Properly aligned and stable well-functioning micromanipulators along with plenty of working area underneath the objective will greatly facilitate sequentially positioning each electrode in a timely manner. After each successful patch, make sure the electrodes from previously established patches have not drifted away from their neuron.

Once the cells have been patched and their membrane and firing properties characterized, inject a depolarizing current step to elicit an action potential in one of the neurons and monitor the postsynaptic cells for a response. Importantly, you must know the reversal potential for the response desired and adjust the holding potential accordingly. When examining GABAergic responses, if the calculated reversal potential is around $-80 \mathrm{mV}$, holding the neuron closer to -55 $\mathrm{mV}$ will maximize the inhibitory response. In some cases, multiple action potentials or a burst of spikes might be needed in the presynaptic neuron to obtain a response in the postsynaptic neuron. In this case, inject a series or train of depolarizing pulses into the presynaptic neuron. Ideally, to clearly determine the synaptic response, at least 50 sweeps (each sweep representing a stimulated presynaptic neuron) will be needed to fully characterize the connection.

To study specific currents, select channel antagonists may be added to isolate the currents of interest. For example, AMPA-sensitive glutamate receptor (-R) antagonist DNQX and NMDA-R 
antagonist DL-AP5 can be used to eliminate excitatory synaptic transmission, and thus limit cellcell communication to primarily inhibitory synapses.

\subsection{Histology and Electron Microscopy}

After the electrophysiological results are obtained, the cortical slices may be immersed in $3 \%$ acrolein/4\% paraformaldehyde in a $0.1 \mathrm{M}$ phosphate-buffered saline at $4{ }^{\circ} \mathrm{C}$ to preserve tissue morphology. After the 24 hour incubation, the slices can be further processed using the avidinbiotin-peroxidase method to elucidate the morphologies of the recorded cells, and then reconstructed using a computerized reconstruction system like Neurolucida. For electron microscopic examination, the slices should be further sectioned and postfixed. To do so, the slice products from the first staining are re-sectioned into $60 \mu \mathrm{m}$ sections, immersed in $1 \% \mathrm{OsO}_{4}$, counterstained with $1 \%$ uranyl acetate, and embedded into resin. From here, ultrathin slices are taken from small areas of interest, specifically to examine putative synaptic bouton sites. From this analysis, identification of inhibitory synapses can be determined by examining details of the synaptic cleft, including: 1) presence of membranes with parallel alignment forming synaptic clefts that are wider in the middle and close up at one or both edges; 2) absence of a prominent postsynaptic density; and 3) presence of multiple flattened synaptic vesicles with at least one docked at the presynaptic membrane.

\section{Exemplar Results and Analysis}

Figure 1 illustrates representative results using the techniques outlined above. A preliminary step in our study of inhibitory interneuronal circuit analysis is the identification of inhibitory synaptic 
connections. By including DNQX and DL-AP5 in the bath solution, the unitary inhibitory postsynaptic currents (UIPSCs) or potentials (UIPSPs) were isolated and measured. After recordings, the axonal arborization of recorded neurons was reconstructed. The reconstruction provided anatomical confirmation of physiologically identified inhibitory synaptic connections, as well as identification of cell type of recorded cells. Based on previously established axonal arborization-based interneuronal classification schemes (Markram et al. 2004; Ascoli et al. 2008; Jiang et al. 2013; Kubota 2014), the Zhu lab could unambiguously classify L1-3 interneurons into 9 general groups, including SBCs and ENGCs in L1, and MaCs, NGCs, BTCs, BPCs, BaC, DBCs and $\mathrm{ChCs}$ in L2/3. Specifically, L1 SBCs have heterogeneous dendritic morphology and a characteristic vertically descending horsetail-like axonal bundle with short side branches. In contrast, L1 ENGCs have their dense axonal arborization elongated horizontally and restricted largely within L1. L2/3 MaCs have their axonal arborization specialized to project mainly towards L1. L2/3 NGCs have their axonal arborization formed into a highly symmetrical and spherical dendritic field. L2/3 BTCs have give rise to two primary dendrites from opposite poles and an axon forming wide vertical and horizontal projections. L2/3 BPCs have an axon commonly emerging from one of the primary dendrites and forming a narrow descending band that crosses multiple layers. $\mathrm{L} 2 / 3 \mathrm{BaCs}$ have a basket-like axonal arborization. L2/3 ChCs have an axon forming characteristic chandelier-like terminals with short vertical rows of boutons. These interneurons can often be identified with direct visual assessment if the majority of their axonal arborization is recovered. The axonal length density analysis can verify the cell type identification since these seven types of L2/3 interneurons differ significantly in their axonal arborization patterns, reflecting presumably the participation of distinct circuit connections. 


\section{Conclusion}

In order to analyze complex behavior at the circuit level, the identification and role of each participating neuron must be known. Because of the vast diversity of interneurons, in their morphology, chemical and electrophysiological characteristics, as well as their distinct synaptic properties and organization, deciphering neuronal circuitry and its effect on behavior have been challenging. Therefore, to analyze the circuits involved in attention, which are known to contain many diverse interneurons, I helped employ the use of a simultaneous quadruple-octuple wholecell recordings method I helped developed. Furthermore, because the testable connectivity pattern, $C$, increases exponentially with the increase of the number of simultaneously recorded neurons, $n$, or $C=4^{n \bullet(n-1) / 2}$, simultaneous quadruple-octuple whole-cell recordings dramatically increase the yield and chance to detect the complex synaptic interconnections. Therefore, analyzing transsynaptic connections of $4-8$ cell-type identified neurons promises to decode the organization of complex cortical inhibitory circuits used in different types of behavior. 


\section{FIGURE LEGEND}

Figure 1. Cortical L1 $\rightarrow \mathrm{L} 2 / 3$ interneuronal circuits.

(A1-3) Images show a typical octuple recording setting.

(B) Reconstruction of L1 SBC (pink), L2/3 BaC (cyan), L2/3 MaC (red), L2/3 BPC (green), L2/3

DBC (blue) and L2/3 BTC (yellow) recorded simultaneously from an acute cortical slice. The double colored dots indicate the putative synaptic contacts based on anatomical reconstruction. The schematic drawing shows symbolically the synaptic connections.

(C) Single action potentials elicited in SBC evoked uIPSPs in postsynaptic BaC, MaC, BPC, DBC and BTC. Scale bars apply to all recording traces with $80 \mathrm{mV}$ and $4 \mathrm{mV}$ bars applied to traces with and without action potentials, respectively. 
Fig. 1

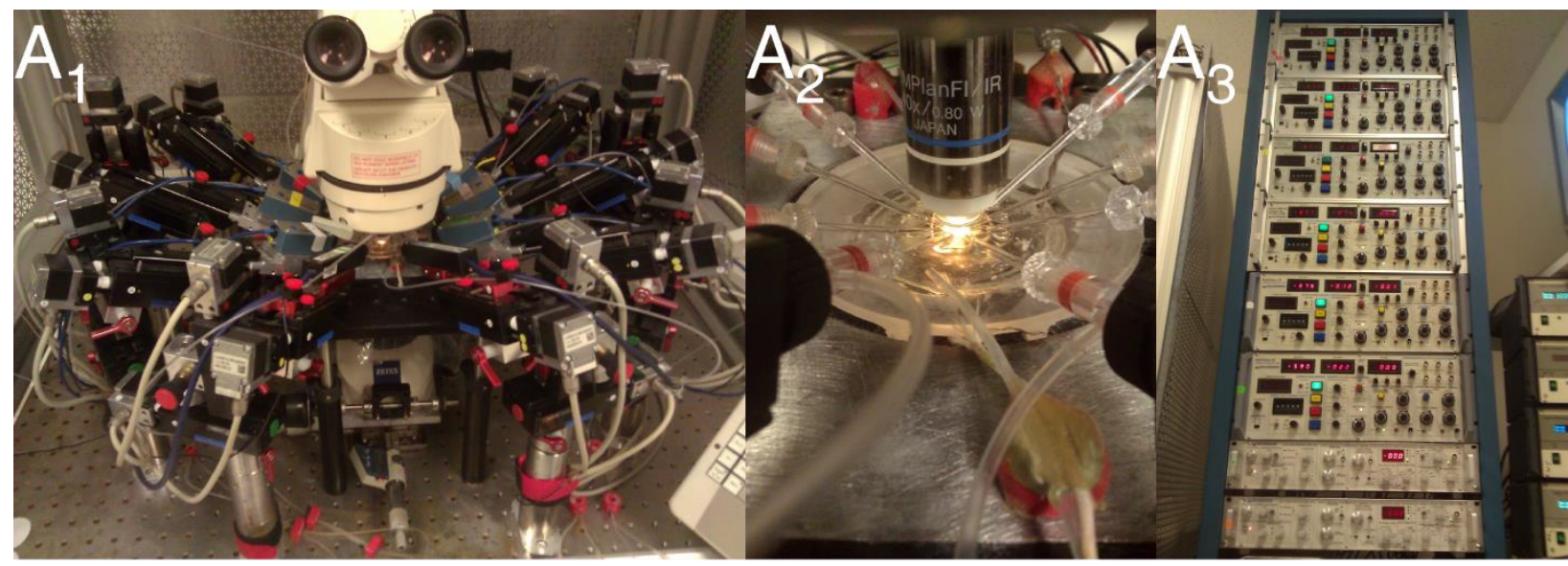

B

L1

L2/3
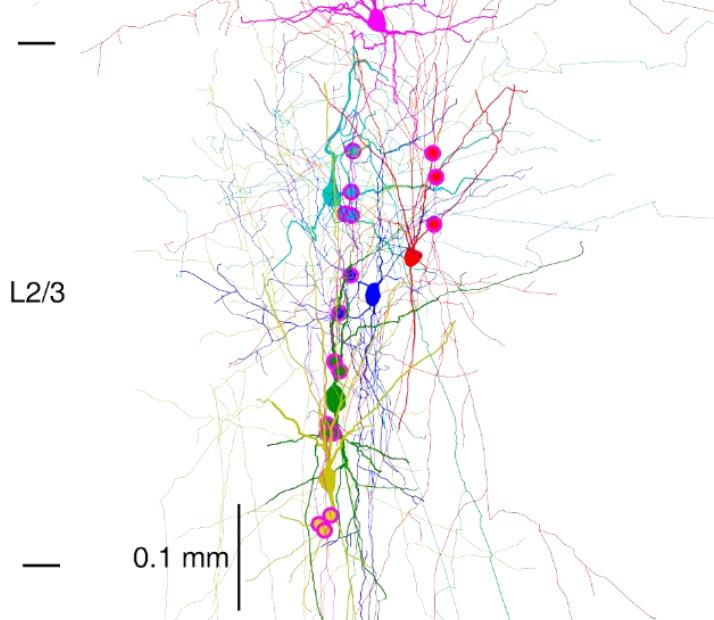

L4

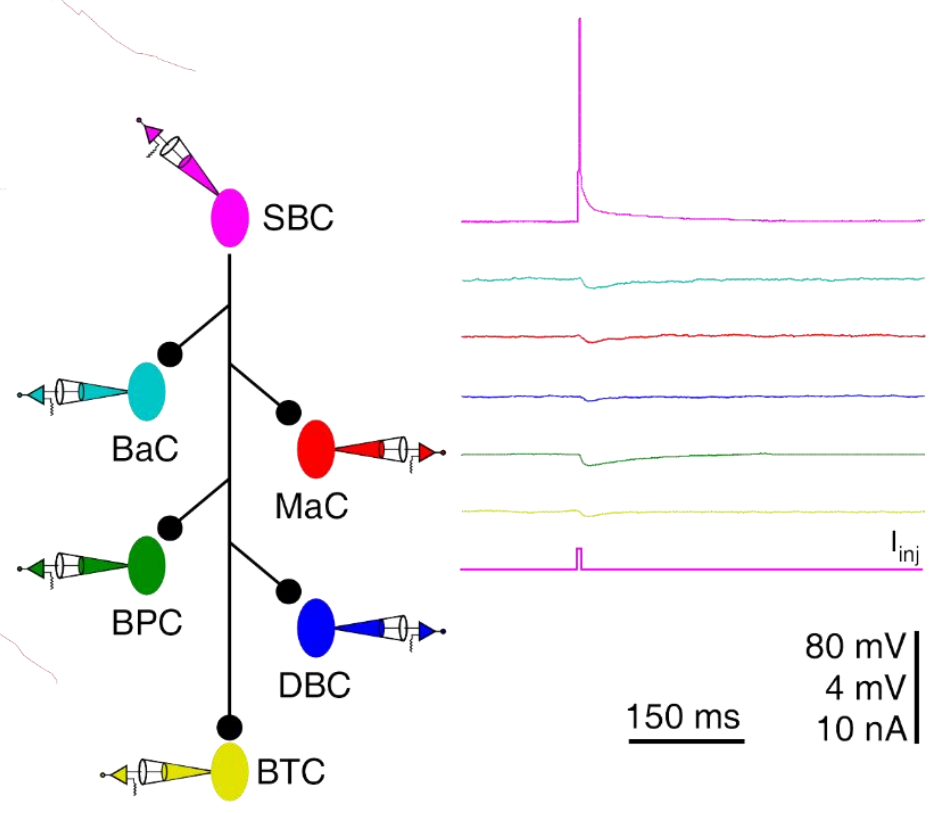

L5-6 


\section{References}

Antic SD (2003) Action potentials in basal and oblique dendrites of rat neocortical pyramidal neurons. The Journal of physiology 550 (Pt 1):35-50. doi:10.1113/jphysiol.2002.033746

Ascoli GA, Alonso-Nanclares L, Anderson SA, Barrionuevo G, Benavides-Piccione R, Burkhalter A, Buzsaki G, Cauli B, Defelipe J, Fairen A, Feldmeyer D, Fishell G, Fregnac Y, Freund TF, Gardner D, Gardner EP, Goldberg JH, Helmstaedter M, Hestrin S, Karube F, Kisvarday ZF, Lambolez B, Lewis DA, Marin O, Markram H, Munoz A, Packer A, Petersen CC, Rockland KS, Rossier J, Rudy B, Somogyi P, Staiger JF, Tamas G, Thomson AM, Toledo-Rodriguez M, Wang Y, West DC, Yuste R (2008) Petilla terminology: nomenclature of features of GABAergic interneurons of the cerebral cortex. Nature reviews Neuroscience 9 (7):557-568

Baluch F, Itti L (2011) Mechanisms of top-down attention. Trends in neurosciences 34 (4):210224. doi:10.1016/j.tins.2011.02.003

Batista-Brito R, Fishell G (2009) The developmental integration of cortical interneurons into a functional network. Curr Top Dev Biol 87:81-118

Cauller LJ, Clancy B, Connors BW (1998) Backward cortical projections to primary somatosensory cortex in rats extend long horizontal axons in layer I. The Journal of comparative neurology 390 (2):297-310

Cauller LJ, Kulics AT (1991) The neural basis of the behaviorally relevant N1 component of the somatosensory-evoked potential in SI cortex of awake monkeys: evidence that backward cortical projections signal conscious touch sensation. Experimental brain research Experimentelle Hirnforschung Experimentation cerebrale 84 (3):607-619 
Christophe E, Roebuck A, Staiger JF, Lavery DJ, Charpak S, Audinat E (2002) Two types of nicotinic receptors mediate an excitation of neocortical layer I interneurons. Journal of neurophysiology 88 (3):1318-1327

Chu Z, Galarreta M, Hestrin S (2003) Synaptic interactions of late-spiking neocortical neurons in layer 1. J Neurosci 23 (1):96-102

Cruikshank SJ, Ahmed OJ, Stevens TR, Patrick SL, Gonzalez AN, Elmaleh M, Connors BW (2012) Thalamic control of layer 1 circuits in prefrontal cortex. J Neurosci 32 (49):1781317823. doi:10.1523/JNEUROSCI.3231-12.2012

DeFelipe J, Farinas I (1992) The pyramidal neuron of the cerebral cortex: morphological and chemical characteristics of the synaptic inputs. Prog Neurobiol 39 (6):563-607

Douglas RJ, Martin KA (2007) Mapping the matrix: the ways of neocortex. Neuron 56 (2):226238

Edwards FA, Konnerth A, Sakmann B, Takahashi T (1989) A thin slice preparation for patch clamp recordings from neurones of the mammalian central nervous system. Pflugers Archiv : European journal of physiology 414 (5):600-612

Geiger JR, Bischofberger J, Vida I, Frobe U, Pfitzinger S, Weber HJ, Haverkampf K, Jonas P (2002) Patch-clamp recording in brain slices with improved slicer technology. Pflugers Archiv : European journal of physiology 443 (3):491-501

Gilbert CD, Sigman M (2007) Brain states: top-down influences in sensory processing. Neuron 54 (5):677-696. doi:10.1016/j.neuron.2007.05.019 
Gilbert CD, Wiesel TN (1983) Functional organization of the visual cortex. Progress in brain research 58:209-218. doi:10.1016/S0079-6123(08)60022-9

Huang ZJ, Di Cristo G, Ango F (2007) Development of GABA innervation in the cerebral and cerebellar cortices. Nature reviews Neuroscience 8 (9):673-686

Jiang X, Wang G, Lee AJ, Stornetta RL, Zhu JJ (2013) The organization of two new cortical interneuronal circuits. Nature neuroscience 16 (2):210-218. doi:10.1038/nn.3305

Johnson RR, Burkhalter A (1997) A polysynaptic feedback circuit in rat visual cortex. J Neurosci $17(18): 7129-7140$

Kastner S, Ungerleider LG (2000) Mechanisms of visual attention in the human cortex. Annual review of neuroscience 23:315-341. doi:10.1146/annurev.neuro.23.1.315

Kubota Y (2014) Untangling GABAergic wiring in the cortical microcircuit. Current opinion in neurobiology 26:7-14

Kubota Y, Shigematsu N, Karube F, Sekigawa A, Kato S, Yamaguchi N, Hirai Y, Morishima M, Kawaguchi Y (2011) Selective coexpression of multiple chemical markers defines discrete populations of neocortical GABAergic neurons. Cereb Cortex 21 (8):1803-1817. doi:10.1093/cercor/bhq252

Kuhn B, Denk W, Bruno RM (2008) In vivo two-photon voltage-sensitive dye imaging reveals top-down control of cortical layers 1 and 2 during wakefulness. Proceedings of the National Academy of Sciences of the United States of America 105 (21):7588-7593. doi:10.1073/pnas.0802462105 
Larkum M (2013) A cellular mechanism for cortical associations: an organizing principle for the cerebral cortex. Trends in neurosciences 36 (3):141-151. doi:10.1016/j.tins.2012.11.006

Larkum ME, Nevian T, Sandler M, Polsky A, Schiller J (2009) Synaptic integration in tuft dendrites of layer 5 pyramidal neurons: a new unifying principle. Science 325 (5941):756760

Larkum ME, Zhu JJ (2002) Signaling of layer 1 and whisker-evoked $\mathrm{Ca}^{2+}$ and $\mathrm{Na}^{+}$action potentials in distal and terminal dendrites of rat neocortical pyramidal neurons in vitro and in vivo. $\mathbf{J}$ Neurosci 22 (16):6991-7005.

Larkum ME, Zhu JJ, Sakmann B (1999) A new cellular mechanism for coupling inputs arriving at different cortical layers. Nature 398 (6725):338-341

Lee AJ, Wang G, Jiang X, Johnson SM, Hoang ET, Lante F, Stornetta RL, Beenhakker MP, Shen Y, Julius Zhu J (2014) Canonical Organization of Layer 1 Neuron-Led Cortical Inhibitory and Disinhibitory Interneuronal Circuits. Cereb Cortex. doi:10.1093/cercor/bhu020

Letzkus JJ, Wolff SB, Meyer EM, Tovote P, Courtin J, Herry C, Luthi A (2011) A disinhibitory microcircuit for associative fear learning in the auditory cortex. Nature 480 (7377):331-335. doi:10.1038/nature10674

Lisman JE (1997) Bursts as a unit of neural information: making unreliable synapses reliable. Trends in neurosciences 20 (1):38-43

Ma J, Yao XH, Fu Y, Yu YC (2013) Development of Layer 1 Neurons in the Mouse Neocortex. Cereb Cortex. doi:10.1093/cercor/bht114 
Markram H, Toledo-Rodriguez M, Wang Y, Gupta A, Silberberg G, Wu C (2004) Interneurons of the neocortical inhibitory system. Nature reviews Neuroscience 5 (10):793-807

Mountcastle VB (1997) The columnar organization of the neocortex. Brain : a journal of neurology 120 (Pt 4):701-722

Muralidhar S, Wang Y, Markram H (2013) Synaptic and cellular organization of layer 1 of the developing rat somatosensory cortex. Frontiers in neuroanatomy 7:52. doi:10.3389/fnana.2013.00052

Pascual-Leone A, Walsh V (2001) Fast backprojections from the motion to the primary visual area necessary for visual awareness. Science 292 (5516):510-512. doi:10.1126/science.1057099

Petreanu L, Mao T, Sternson SM, Svoboda K (2009) The subcellular organization of neocortical excitatory connections. Nature 457 (7233):1142-1145

Purushothaman G, Marion R, Li K, Casagrande VA (2012) Gating and control of primary visual cortex by pulvinar. Nature neuroscience 15 (6):905-912. doi:10.1038/nn.3106

Reyes A (2001) Influence of dendritic conductances on the input-output properties of neurons. Annual review of neuroscience 24:653-675

Robinson DL, Petersen SE (1992) The pulvinar and visual salience. Trends in neurosciences 15 (4):127-132

Rockland KS, Pandya DN (1979) Laminar origins and terminations of cortical connections of the occipital lobe in the rhesus monkey. Brain research 179 (1):3-20

Schiller J, Schiller Y, Stuart G, Sakmann B (1997) Calcium action potentials restricted to distal apical dendrites of rat neocortical pyramidal neurons. J Physiol (Lond) 505 (Pt 3):605-616 
Sjostrom PJ, Rancz EA, Roth A, Hausser M (2008) Dendritic excitability and synaptic plasticity. Physiological reviews 88 (2):769-840

Spruston N (2008) Pyramidal neurons: dendritic structure and synaptic integration. Nature reviews Neuroscience 9 (3):206-221. doi:10.1038/nrn2286

Tomita H, Ohbayashi M, Nakahara K, Hasegawa I, Miyashita Y (1999) Top-down signal from prefrontal cortex in executive control of memory retrieval. Nature 401 (6754):699-703. doi:10.1038/44372

van Boxtel JJ, Tsuchiya N, Koch C (2010) Consciousness and attention: on sufficiency and necessity. Frontiers in psychology 1:217. doi:10.3389/fpsyg.2010.00217

van Gaal S, Lamme VA (2012) Unconscious high-level information processing: implication for neurobiological theories of consciousness. The Neuroscientist : a review journal bringing neurobiology, neurology and psychiatry 18 (3):287-301. doi:10.1177/1073858411404079

Waters J, Larkum M, Sakmann B, Helmchen F (2003) Supralinear Ca2+ influx into dendritic tufts of layer 2/3 neocortical pyramidal neurons in vitro and in vivo. J Neurosci 23 (24):85588567

Wozny C, Williams SR (2011) Specificity of synaptic connectivity between layer 1 inhibitory interneurons and layer 2/3 pyramidal neurons in the rat neocortex. Cereb Cortex 21 (8):18181826. doi:10.1093/cercor/bhq257

Xu NL, Harnett MT, Williams SR, Huber D, O'Connor DH, Svoboda K, Magee JC (2012) Nonlinear dendritic integration of sensory and motor input during an active sensing task. Nature 492 (7428):247-251. doi:10.1038/nature11601 
Yuen EY, Yan Z (2009) Dopamine D4 receptors regulate AMPA receptor trafficking and glutamatergic transmission in GABAergic interneurons of prefrontal cortex. J Neurosci 29 (2):550-562

Zhu JJ (2000) Maturation of layer 5 neocortical pyramidal neurons: amplifying salient layer 1 and layer 4 inputs by $\mathrm{Ca}^{2+}$ action potentials in adult rat tuft dendrites. $\mathrm{J}$ Physiol (Lond) $526(\mathrm{Pt}$ 3):571-587

Zhu JJ (2009) Activity level-dependent synapse-specific AMPA receptor trafficking regulates transmission kinetics. J Neurosci 29 (19):6320-6335

Zhu JJ, Connors BW (1999) Intrinsic firing patterns and whisker-evoked synaptic responses of neurons in the rat barrel cortex. Journal of neurophysiology 81 (3):1171-1183.

Zhu Y, Zhu JJ (2004) Rapid arrival and integration of ascending sensory information in layer 1 nonpyramidal neurons and tuft dendrites of layer 5 pyramidal neurons of the neocortex. $\mathbf{J}$ Neurosci 24 (6):1272-1279 


\section{CHAPTER 4}

Disclaimer: Reconstructions were performed by another lab member and are not meant to correspond to the actual cell morphologies

Examination of a Population of Reconstructed Inhibitory Interneurons in Cortical Layer 6 


\begin{abstract}
Cortical layer 6 (L6) is the most enigmatic layer of the cortex. Interneurons in L6 are particularly poorly understood. In the present study, I used the simultaneous multiple whole-cell recording system to examine 15 L6 inhibitory interneurons from the sensorimotor cortices of 15 rats. My aim was to analyze the physiological and anatomical properties and determine what relationships exist between these properties. After examination of the intrinsic electrophysiological properties of these cells, I grouped the interneurons into fast-spiking (FS) and non-FS interneurons (NFS). FS interneurons in other cortical layers display physiological properties that allow for fast synaptic transmission. To test this in L6, I grouped these cells into FS and NFS cells and then compared their intrinsic and synaptic physiological properties. I found that FS interneurons fired at higher frequencies and had shorter action potential half-widths. I then examined the differences in synaptic physiological properties between FS and NFS interneurons. Results of these analyses showed a significant difference in the synaptic rise time of unitary inhibitory postsynaptic potentials, but no significant differences in the slope $(\Delta \mathrm{V} / \Delta \mathrm{t})$ of the response, the amplitude, the response half-width or the decay tau. I then examined the morphologies of these interneurons. I found no significant differences in the morphologies between FS and NFS interneurons. Lastly, I examined the relationship between the synaptic physiological properties and the morphological properties. I found that synaptic response decay constant positively correlates with the mean distance of putative synaptic contact sites from the soma.
\end{abstract}




\section{Introduction}

The cerebral cortex is composed of different layers, each having a specific role in the cortical microcircuit. L6 is the deepest layer, comprising nearly one third of the cerebral cortex (Gabbott, Dickie, Vaid, Headlam, \& Bacon, 1997; Van Eden \& Uylings, 1985). The least understood of the cortical layers, L6 has been described as the enigmatic layer. Research in the cytology of L6 has largely focused on excitatory neurons. These studies show that L6 is a principal cortical output layer and is critical in regulating sensory information due to its reciprocal connectivity to specific thalamic nuclei (Briggs, 2010; Lam \& Sherman, 2009; Radnikow, Qi, \& Feldmeyer, 2015; Thomson, 2010; Thomson \& Lamy, 2007; West, Mercer, Kirchhecker, Morris, \& Thomson, 2005).

L6 interneurons are not well understood. Significant effort has been made to group these neurons based on their morphologies (Arzt, Sakmann, \& Meyer, 2017; Cajal, 1911; Chen, Abrams, Pinhas, \& Brumberg, 2009; Ferrer, Fabregues, \& Condom, 1986; Kumar \& Ohana, 2008; Markram et al., 2015; O'Leary, 1941; Prieto \& Winer, 1999; Tömböl, 1984). L6 interneurons characterized as "fast spiking" are thought to have a critical role in brain functions (Bortone, Olsen, \& Scanziani, 2014; Cruikshank, Urabe, Nurmikko, \& Connors, 2010; Olsen, Bortone, Adesnik, \& Scanziani, 2012). These cells are strongly innervated by thalamic inputs and are suggested to be the main drivers for rapid feed-forward inhibition in L6 (Cruikshank et al., 2010). However, the morphologies of L6 FS interneurons are poorly understood.

While the physiological differences between FS and NFS interneurons in L6 are not clear, studies in other cortical layers indicate that FS cells have faster action potential half-widths and 
fire at higher frequencies that NFS cells (Rudy \& McBain, 2001). In addition, these cells have lower input resistances and faster membrane time constants, which enables fast synaptic connectivity (Rudy, Fishell, Lee, \& Hjerling-Leffler, 2011). Furthermore, FS interneurons demonstrate faster synaptic inhibition that is thought to facilitate coincidence detection ( $\mathrm{Hu}, \mathrm{Gan}$, \& Jonas, 2014; Pouille \& Scanziani, 2001). These physiological differences are the bases for proposed microcircuits within the layer L2/3 (Avermann, Tomm, Mateo, Gerstner, \& Petersen, 2012).

In this study, I examined a population of reconstructed L6 interneurons. First, I tested the hypothesis that L6 FS interneurons form a distinct subgroup from NFS interneurons based on their intrinsic electrophysiological properties. I then tested the hypothesis that L6 FS interneurons mediate significantly faster synaptic responses. In addition, I tested the hypothesis that FS interneurons significantly differ from NFS interneurons in their morphology. Lastly, I examined the relationship between the synaptic physiologies and the morphologies of the L6 reconstructed interneurons. 


\section{Methods}

\section{Ethical approval}

All experiments described were conducted in accordance with the U.S. Public Health Service Policy on Humane Care and Use of Laboratory Animals and were approved by the Institutional Animal Care and Use Committee of the University of Virginia.

\section{Animals}

Fifteen both male and female Sprang Dawley rats postnatal 20 days or older were used to ensure examination of mature and stabilized cortical inhibitory neurons and circuits (Batista-Brito \& Fishell, 2009; Huang, Di Cristo, \& Ango, 2007). Some experiments were performed using transgenic Venus-expressing rats in order to target interneurons (Uematsu et al., 2007).

\section{Brain slice preparation and electrophysiology}

The procedure for slice preparation and electrophysiology followed the one previous described (Wang et al., 2015; Wyskiel, Larry, Jiang, Wang, \& Zhu, 2016). Briefly, both male and female rats were anesthetized and then decapitated. Brains were removed and cooled in standard ACSF solution (composition in mM: $125 \mathrm{NaCl}, 2.5 \mathrm{KCl}, 1 \mathrm{MgCl}_{2}, 2 \mathrm{CaCl}_{2}, 1.25 \mathrm{NaH}_{2} \mathrm{PO}_{4}, 25$ $\mathrm{NaHCO}_{3}$ and 25 dextrose) saturated with carbogen with a pH 7.4. Acute cortical slices of 300$350 \mu \mathrm{m}$ thickness were obtained using a microslicer. Slices were allowed to recover in a $37.0 \pm$ $0.5^{\circ} \mathrm{C}$ water bath for at least 30 minutes. Parasagittal slices were transferred one at a time to a recording chamber on the stage of an upright Zeiss microscope. Slices were held in place on the chamber by a platinum and nylon grid and continuously perfused with standard ACSF solution at $32 \pm 1{ }^{\circ} \mathrm{C}$ bubbled to saturation with $95 \% \mathrm{O}_{2}$ and $5 \% \mathrm{CO}_{2}$. Patch electrodes were pulled from 2.0 
mm o.d. borosilicate glass using a Flaming-Brown model P-97 horizontal puller (Sutter Instruments, Novato, CA) and filled with a potassium-based intracellular solution (composition in mM: 120 potassium gluconate, $10 \mathrm{HEPES}, 4 \mathrm{KCl}, 4 \mathrm{MgATP}, 0.3 \mathrm{Na}_{3} \mathrm{GTP}, 10$ disodium phosphocreatine and $0.5 \%$ biocytin). The resistance of the pipette was generally between 3-7 $\mathrm{M} \Omega$.

\section{Electrophysiological parameters and data analysis}

Electrophysiological parameters were analyzed with the assistance of custom software in IGOR and Excel. The membrane time constant $\left(\tau_{\mathrm{m}}\right)$ and input resistance were estimated based on the voltage deflection elicited by $600 \mathrm{~ms}$ long hyperpolarizing current pulses. Action potential amplitude and half-width were calculated based on the first spike elicited by depolarizing current steps. The action potential amplitude was measured from action potential threshold to the peak. The action potential half-width corresponds to the duration of the action potential at halfamplitude. A fast afterhyperpolarization (AHP) following the spike was measured from its peak amplitude to action potential threshold. Adaptation index was calculated based on three or more action potentials. Firing rates were assessed near threshold using the last interspike interval. Care was taken to give best fits or estimates of the parameters, however some parameters could not be obtained sufficiently for every cell and were not used for some analyses. Analysis of the synaptic properties were obtained based on the averaged response elicited by presynaptic stimulation of single action potentials. The synaptic latency was calculated from the peak of the action potential of the presynaptic neuron to the onset of the response in the postsynaptic neuron. The uIPSP slope, $\Delta \mathrm{V} / \Delta \mathrm{t}$, was calculated as uIPSP amplitude $* 0.8 /(10-90 \%$ rise time $)$. 


\section{Classification of cells}

Cells were described as inhibitory interneurons due to eliciting an apparent inhibitory response in a L6 postsynaptic neuron. Interneurons were grouped as "fast spiking" (FS) based on their firing frequency of $>40 \mathrm{~Hz}$ following previous classifications and observations (Karube, Kubota, \& Kawaguchi, 2004; Kawaguchi \& Kondo, 2002). Two cells displayed a firing rate of $39 \mathrm{~Hz}$ and were classified as FS due to their lack of adaption and/or short action potential half-width. Action potential half-widths were not solely used as some L6 interneurons with short half-widths were adapting and did not fire at high frequencies, clearly differentiating them from FS cells. Pyramidal neurons were defined as having a pyramidal soma identified under the light microscope. Significant differences between inhibitory interneurons derived from wild type and YFP-expressing cells derived from transgenic animals were found when examining their intrinsic electrophysiological properties; however, no differences were found when examining synaptic physiological properties (data not shown). This may be due to recording from YFP-expressing cells that were not interneurons which is expected to a small extent with this transgenic animal (Uematsu et al., 2007). Another possibility is that the wild-type L6 inhibitory interneurons examined are not representative of all L6 inhibitory interneurons due to only recording within L6.

\section{Cluster analyses}

Cluster analyses was used for visualization of the data and not for dividing the population into subgroups. This was performed using a custom code written in the R programming language. The parameters used for the cluster analyses were standardized and based on the action potential half-width, AHP, firing rate near threshold, adaptation, membrane tau, input resistance, action 
potential threshold, action potential amplitude and the resting membrane potential. Parameters from two interneurons could not be used and, therefore, 13 cells were used for cluster analyses. Model-based clustering used the mclust package. Hierarchical clustering used the hclust R function and the numbers correspond to individual cells. K-means clustering used the kmeans R function and two groups were proposed.

\section{Immunohistochemistry and morphological analyses of L6a examined interneurons} Immunostaining and reconstructing cells followed methods previously described (Wang et al., 2015). Briefly, slices were fixed for at least 24 hours and then were processed using the avidinbiotin-peroxidase method. Slices were reconstructed using the computerized reconstruction system Neurolucida with a 100x oil-immersion objective. Morphological analyses of the L6a interneurons were based on the reconstructions. Using Neuroexplorer within the Neurolucida software, Sholl analysis was performed using a series of $20 \mu \mathrm{m}$ radii circles with the soma at the center. The Sholl critical value corresponds to the sphere in which the highest number of intersections were found. Putative contact sites were estimated under the light microscope as an apposition of an axon terminal with a neuron within the same plane.

\section{Statistical analysis}

Statistical analysis was calculated using Excel. Data was reported as mean \pm SEM. Statistical significance between two groups was determined using a two-tailed, unpaired t-test.

\section{Results}

Membrane and firing properties of L6 interneurons 
The intrinsic properties of interneurons help determine the functional role in neuronal circuitry. Therefore, I first sought to examine the membrane and firing properties of these reconstructed L6 interneurons. Using whole-cell electrophysiological recordings from L6 inhibitory interneurons in cortical brain slices, a series of hyperpolarizing and depolarizing current pulses were used to examine passive and active membrane properties. Examples of two L6 interneurons are shown in Figure 1. Overall, these L6 interneurons had an average resting membrane potential of $-69.2 \pm$ $1.03 \mathrm{mV}$. Their mean input resistance at steady-state was $198.5 \pm 19.1 \mathrm{M} \Omega$ and their membrane time constant was $10.4 \pm 1.3 \mathrm{~ms}$. The mean action potential amplitude was $58.9 \pm 3.5 \mathrm{mV}$, with an average half-width of $0.70 \pm 0.06 \mathrm{~ms}$. The mean AHP was $-13.6 \pm 1.1 \mathrm{mV}$. Examination of the spiking revealed heterogeneity in their spiking behavior, as illustrated in Figure 1 for two interneurons. Six interneurons demonstrated high firing rates in response to depolarizing current pulses and were classified as FS interneurons (see methods). The remaining interneurons were found to be adapting or slightly adapting $(n=3)$, or "regular spiking" $(n=5)$ that produced nonadapting lower-frequency spiking in response to prolonged depolarizing current injections. The firing frequencies of FS interneurons averaged $48.5 \pm 5.4 \mathrm{~Hz}$ and ranged from $39.1-70.8 \mathrm{~Hz}$ at near threshold frequencies. The non-FS (NFS) averaged $11.9 \pm 2.5 \mathrm{~Hz}$ and ranged from 2.5-21.5 Hz. The significance in firing rate between the two groups was high $(\mathrm{p}<0.0005)$.

I next examined whether differences in other intrinsic properties could be found between FS and NFS interneurons. Results of these analyses are summarized in Figure 2. FS inhibitory interneurons had an average resting membrane potential of $-71.5 \pm 1.4 \mathrm{mV}$. Their mean input resistance at steady-state was $173.2 \pm 35.3 \mathrm{M} \Omega$ and their membrane time constant was $9.25 \pm$ $1.74 \mathrm{~ms}$. The mean action potential amplitude was $54.0 \pm 4.1 \mathrm{mV}$, with an average half-width of 
$0.56 \pm 0.06 \mathrm{~ms}$. The mean AHP was $-14.5 \pm 2.0 \mathrm{mV}$. NFS inhibitory interneurons had an average resting membrane potential of $-68 \pm 1.4 \mathrm{mV}$, an input resistance of $213.4 \pm 25.1 \mathrm{M} \Omega$ and a membrane time constant of $12.2 \pm 2.0 \mathrm{~ms}$. The mean action potential amplitude was $63.0 \pm 6.6$ $\mathrm{mV}$ with a mean half-width of $0.82 \pm 0.09 \mathrm{~ms}$. The mean AHP was $-12.9 \pm 1.6 \mathrm{mV}$. Along with the firing rate, a significant difference was found in the action potential half-width between FS and NFS interneurons. FS interneurons has a significantly shorter action potential half-widths (FS: $0.56 \pm 0.06 \mathrm{~ms}$; NFS: $0.82 \pm 0.09 \mathrm{~ms}, \mathrm{p}<0.05$ ). Notably, 2 NFS cells had short action potential half-widths. One was adapting (action potential half-width: $0.59 \mathrm{~ms}$; AI: 2.9) and is illustrated in Figure 1A (right trace). The other cell displayed regular spiking (action potential half-width: $0.46 \mathrm{~ms}$; firing rate: $8.5 \mathrm{~Hz}$ ) and is illustrated in Figure 4B. No significant differences between FS and NFS interneurons were found in the resting membrane potential, membrane tau, input resistance, AHP, action potential threshold, action potential amplitude or adaptation index.

\section{Cluster analyses of the reconstructed L6 interneurons based on intrinsic electrophysiological properties}

Cluster analyses can help with visualization of the data and, if using an appropriate number of cells and parameters, can segregate the population into possibly meaningful subgroups. Here, I used cluster analyses for visualization due to the smaller sample size. Using the membrane and firing properties, I performed cluster analyses on 13 of the 15 L6 interneurons (see methods). I used model-based clustering (see Fig 3A) and then hierarchical clustering (Fig 3B). To segregate the population into 2 groups, I used K-means clustering and proposed 2 groups. Results of this

analysis are shown in Figure 3C. The cells segregated using this method closely resembled those grouped by firing frequency. 


\section{Synaptic responses in postsynaptic neurons elicited by L6 inhibitory interneurons}

I next examined whether these 2 subgroups differed significantly in their synaptic physiology. To facilitate finding connections, I used simultaneous multiple whole-cell electrophysiological recordings. As shown in Figure 4, these interneurons elicited uIPSPs in postsynaptic neurons. To examine the properties of uIPSPs in postsynaptic neurons, depolarizing current was injected into L6 interneurons in order to elicit single action potentials. Average uIPSP latencies, $10-90 \%$ rise times, amplitudes, widths at half amplitude and decay values were measured and are summarized for FS and NFS interneurons in Table 1. A significant difference in the 10-90\% rise times was found. FS interneurons elicited uIPSPs with shorter rise times recorded in postsynaptically connected cells. However, to determine whether this difference results from differences in uIPSP amplitude, I examined the uIPSP slope, $\Delta \mathrm{V} / \Delta \mathrm{t}$. Analysis of this property showed no significant differences between FS and NFS interneurons. No significant differences were found when pyramidal neurons were the only postsynaptic neuron analyzed. FS cells produced an averaged uIPSP amplitude of $-1.4 \pm 0.4 \mathrm{mV}(\mathrm{n}=3)$, latency of $0.3 \pm 0.2 \mathrm{~ms}(\mathrm{n}=5), 10-90 \%$ rise time of $4.6 \pm 1.7 \mathrm{~ms}(\mathrm{n}=3)$, half-width of $48.7 \pm 16.6 \mathrm{~ms}(\mathrm{n}=3)$, and a decay tau of $57.3 \pm 19.6 \mathrm{~ms}(\mathrm{n}=$

3). IPSPs from NFS cells averaged $-0.5 \pm 0.2 \mathrm{mV}$ in amplitude $(\mathrm{n}=4)$, latency of $0.9 \pm 0.6 \mathrm{~ms}(\mathrm{n}$ $=2), 10-90 \%$ rise time of $8.9 \pm 3.0 \mathrm{~ms}(\mathrm{n}=3)$, half-width of $64.6 \pm 10.0 \mathrm{~ms}(\mathrm{n}=4)$, and a decay tau of $68.6 \pm 16.6 \mathrm{~ms}(\mathrm{n}=4)$.

\section{Morphological analysis of reconstructed L6 interneurons}

I began the morphological analyses with examination of the cell somas. Somas of both FS and NFS interneurons were predominantly small and round (see Fig. 5A). The mean horizontal 
diameters were $15.9 \pm 1.2$ for FS cells $(n=5)$ and $16.5 \pm 1.0$ for NFS cells $(n=7)$; and the mean vertical diameters were 19.5 \pm 2.1 for FS cells $(n=5)$ and $20.5 \pm 2.0$ for NFS cells $(n=7)$. No significant differences between the somas were found. Three somas were unable to be adequately measured due to their placement overlapping another cell. Next, I examined the dendrites of the L6 reconstructed interneurons. As shown in Figure 5B, the number of primary dendrites was similar for the two subgroups: $6.0 \pm 0.9$ for FS cells $(n=6)$ and $5.7 \pm 0.8$ for NFS cells $(n=9)$.

Due to simultaneously recording and biocytin filling of neurons in close proximity, accurate morphological analyses of the axonal and dendritic arbors were hampered. However, 13 of the reconstructed interneurons could be properly analyzed for their dendritic and axonal arbor morphologies. Based on the dendritic arborizations, most cells were multipolar; however, one appeared to be bipolar. Dendrites were found to typically radiate away and terminated within L6 or in L5b. Some cells $(n=3)$ had what appeared to be a prominent dendrite that extended into L5a or L2/3. The analyses of the dendritic arbors of 5 FS interneurons and 8 NFS interneurons are shown in Figures 5C and 5D. Examples of the Sholl analyses for a FS and NFS interneuron are shown in Figure 6. The dendritic arbors of the L6 cells averaged $22.3 \pm 2.7$ nodes $(n=13)$. The average Sholl critical value was $16.2 \pm 1.8$, which corresponded to a Sholl radius of $68.5 \pm$ 18.57 microns $(n=13)$. The dendritic arbors of FS L6 examined cells averaged $22.6 \pm 3.0$ nodes $(n=5)$. The average Sholl critical value was $13.8 \pm 1.8$, which corresponded to a Sholl radius of $104 \pm 57.5$ microns $(n=5)$. The dendritic arbors of NFS L6 examined cells averaged $22.1 \pm 8.0$ nodes $(\mathrm{n}=8)$. The average Sholl critical value was $17.8 \pm 5.03$, which corresponded to a Sholl radius of $46.3 \pm 15.4$ microns $(n=8)$. Overall, in the analyses of the dendritic arbors, no 
significant differences between FS and NFS were found in either the Sholl analyses or examinations of the nodes.

The axonal arborizations of the examined L6 cells typically were found within or near L6; however, some axons were found to extend across layers and columns. Examination of the biocytin staining of the cells under the light microscope and the reconstructions indicated that none of the cells appeared to form a basket-like synapsis around the postsynaptic neuron. Therefore, no cell met the criterion to be classified as a basket cell (BC). However, extensive axonal branching in some cells was apparent. Analyses of the axonal arbors of the reconstructed L6 interneurons is shown in Figures 5E and 5F. The axonal arbors of the L6 examined cells averaged $35.5 \pm 6.7$ nodes $(n=13)$. The average Sholl critical value was $17.5 \pm 2.1$, which corresponded to a Sholl radius of $122.3 \pm 13.8$ microns $(\mathrm{n}=13)$. The axonal arbors of FS L6 examined cells averaged $28.6 \pm 7.8$ nodes $(n=5)$. The average Sholl critical value was $17.2 \pm$ 3.1, which corresponded to a Sholl radius of $144.0 \pm 20.4$ microns $(n=5)$. The axonal arbors of NFS L6 examined cells averaged $39.8 \pm 9.8$ nodes $(n=8)$. The average Sholl critical value was $17.6 \pm 2.9$, which corresponded to a Sholl radius of $108.8 \pm 17.7$ microns $(n=8)$. Overall, in the analyses of the axonal arbors, no significant differences between FS and NFS were found in either the Sholl analyses or examinations of the nodes.

Examination of the contact site(s) on postsynaptic neurons is thought to help in classifying interneurons (Han, Buhl, Lörinczi, \& Somogyi, 1993; Somogyi, Tamas, Lujan, \& Buhl, 1998). Therefore, I next sought to examine the putative contacts. Examination under the light microscope of 18 pairs of interneurons and postsynaptic neurons in L6 revealed 60 putative 
contact sites. Examples of labeled putative contact sites on the reconstructed cells are shown in Figure 7. Interestingly, connections on the soma were found with only one pair. The average distance from the soma was found to be $66.80 \pm 8.78$ microns. Most contact sites were observed on basal dendrites or collaterals from the apical dendrite. Connected pairs with FS cells averaged $3.9 \pm 0.7$ putative contact sites ( $n=8$ pairs), and the average distance from the soma was found to be $72.5 \pm 9.1$ microns. Connected pairs with NFS cells averaged $2.9 \pm 0.5$ putative contact sites $(n=10$ pairs $)$, and the average distance from the soma was found to be $47.6 \pm 9.2$ microns. No significant differences was found between FS and NFS interneurons in their number of contact sites. However, the distance form soma to contact sites was significantly shorter for NFS interneurons than for FS interneurons $(\mathrm{p}<0.05)$.

\section{Examination of the relationship between synaptic physiological and anatomical properties}

My findings above sought to examine the relationship between the spiking of L6 interneurons and their synaptic physiological and anatomical properties. I next sought to determine if there is a correlation between the synaptic physiological properties and the anatomical properties. First, I examined the relationship between the uIPSP amplitude and the number of putative contact sites on the postsynaptic neuron as shown in Figure 8A. No statistically significant correlation was found $(\mathrm{r}=-0.24 ; \mathrm{p}>0.05 ; \mathrm{n}=10)$. This may be due to an inability to accurately identify contact sites under the light microscope. However, other factors such as differences in release probability may affect the averaged amplitude. Next, I examined the kinetics of the uIPSP verses the distance from the soma. Synaptic connections on more distal dendrites can result in slower uIPSP kinetics recorded at the soma due to electrotonic filtering (Rall, 1967). In addition, uIPSP kinetics can also be affected by the activation of different $\mathrm{GABA}_{\mathrm{A}}$ receptors (Pearce, 1993; 
Wisden, Laurie, Monyer, \& Seeburg, 1992). However, differences in channel properties along the dendrite may also play a role (Häusser, Spruston, \& Stuart, 2000). As shown in Figure 8B, analysis of the uIPSP rise time verses the putative contact site averaged distance from the soma resulted in no statistically significant correlation $(\mathrm{r}=-0.42 ; \mathrm{p}>0.05 ; \mathrm{n}=10)$. To determine whether the UIPSP amplitudes affected this relationship, I examined the slope of the uIPSP at the rising phase (Fig. 8C). Results from the examination of the relationship between the IPSP slope and the putative contact site averaged distance from the soma found no significant correlation ( $\mathrm{r}$ $=0.15 ; \mathrm{p}>0.05 ; \mathrm{n}=10)$. In addition, I examined the uIPSP decay time constant verses the averaged distance from the soma (Fig. 8D). A positive statistically significant correlation was found $(\mathrm{r}=0.65 ; \mathrm{p}>0.05 ; \mathrm{n}=10)$. The more distant the mean distance from the soma resulted in a longer uIPSP decay.

\section{Discussion}

In the present study, I examined a population of reconstructed L6 interneurons. Examination of their firing properties revealed a portion of the cells were FS interneurons. I then tested the hypothesis that FS and NFS interneurons differ in their intrinsic electrophysiological properties. I found that by grouping the interneurons based on their firing rates, FS interneurons significantly differ from NFS interneurons in their AP half-widths. I then tested the hypothesis that FS and NFS interneurons differ in their synaptic physiological properties. Examination of postsynaptic responses elicited by and the morphologies showed a significant difference in the synaptic rise time of the uIPSP, but no significant differences in the slope $(\Delta \mathrm{V} / \Delta \mathrm{t})$ of the response, the amplitude, the response half-width or the decay tau. I then tested the hypothesis that FS and NFS interneurons differ in their morphological properties. Results from this analyses revealed no 
significant differences between these 2 subgroups. Lastly, I examined the relationship between the synaptic physiological properties and the properties obtained through anatomical analyses. I find that the decay of the synaptic response mediated by L6 interneurons positively correlates with the mean distance of the putative synaptic contact sites from the soma.

There were several other limitations to this study. Substantial changes in the intrinsic electrophysiological and synaptic properties, as well the morphologies, occur early in development, including for BCs (Doischer et al., 2008). However, these changes are most pronounced up to the third post-natal week (Doischer et al., 2008). For FS cells, the intrinsic electrophysiological properties are rapid early in development and should be distinguished from NFS by P20 (Goldberg et al., 2010; Miller, Okaty, Kato, \& Nelson, 2011; Okaty, Miller, Sugino, Hempel, \& Nelson, 2009). For example, one study found little to no changes in the membrane and firing properties of FS cells after P18 in mice (Goldberg et al., 2010). However, some small changes later have been indicated (Miller et al., 2011; Okaty et al., 2009).

FS cells were classified based on their firing frequency of $>40 \mathrm{~Hz}$, following previous classifications and observations (Karube et al., 2004; Kawaguchi \& Kondo, 2002). However, this may not adequately distinguish the cells. In addition, FS cells have been classified in different ways. Avermann et al., 2012 used action potential half-width alone to classify L2/3 interneurons in juvenile transgenic mice as FS and NFS (Avermann et al., 2012). In their study, a clear distinction in this property was found with the action potential half-widths of FS interneurons ranging up to $0.65 \mathrm{~ms}$, while NFS interneurons all exceeded $0.85 \mathrm{~ms}$. The cells studied here in L6 did not show distinct populations based on action potential half-widths. Furthermore, I found 
some cells with very low action potential half-widths that did not fire at high frequencies, and in one case was highly adapting.

In this study, I defined BCs as cells that arrange their synapses in a "basket" formation around the postsynaptic neuron observed under the light microscope. However, the formation of "baskets" is typically a result of multiple BCs synapses on the soma of a postsynaptic neuron (DeFelipe, Hendry, \& Jones, 1986; Marin-Padilla, 1969; Somogyi, Kisvarday, Martin, \& Whitteridge, 1983; Somogyi \& Soltesz, 1986; White \& Keller, 1989). Identify BCs by examining their perisomatic synapses using electron microscopy has been used by some researchers as a criteria (T. Freund, Maglóczky, Soltesz, \& Somogyi, 1986; E. H. Jones, SHC, 1984; Kisvárday, 1992; Somogyi \& Soltesz, 1986). BCs form a larger number of perisomatic synapses compared to other GABAergic interneuron subtypes. Therefore, the use of electron microscopy may be needed. Future studies will need to further test the merit of interneuron classification methods in L6.

Significant research has focused on FS interneurons (Hu et al., 2014). These interneurons are specialized to allow for fast synaptic communication. Their function has been implicated in many brain processes and their dysfunction has been implicated in brain disorders (Cardin et al., 2009; Hu et al., 2014; Lewis, Curley, Glausier, \& Volk, 2012). Studies suggest FS interneurons in L6 also have a significant role in brain functions (Bortone et al., 2014; Olsen et al., 2012). Future studies examining these cells may help uncover whether these cells can be grouped further based on their morphologies. 
This preliminary data indicates that L6 interneurons may form subgroups based upon their firing properties. However, whether FS and NFS differ significantly in their synaptic physiological or anatomical properties is not clear. A study examining a larger population of L6 interneurons may be necessary. In this case, cluster analysis to segregate the population may be appropriate and meaningful. Additional properties, such as the excitatory responses recorded in L6 interneurons, should also be examined. Refinement of the criteria for interneuron subtype classifications should be employed. Overall, a better understanding of the relationships between the physiological and anatomical properties of L6 interneurons will facilitate classifying L6 interneurons and aid in deciphering interneuronal circuitry in L6.

\section{Acknowledgements}

Sudip Pandit provided some of the data and analyses for this study. Joe Ford provided the cluster analysis code. 


\section{References}

Arzt, M., Sakmann, B., \& Meyer, H. S. (2017). Anatomical Correlates of Local, Translaminar, and Transcolumnar Inhibition by Layer 6 GABAergic Interneurons in Somatosensory Cortex. Cerebral Cortex, 1-12.

Avermann, M., Tomm, C., Mateo, C., Gerstner, W., \& Petersen, C. C. (2012). Microcircuits of excitatory and inhibitory neurons in layer $2 / 3$ of mouse barrel cortex. Journal of Neurophysiology, 107(11), 3116-3134.

Batista-Brito, R., \& Fishell, G. (2009). The developmental integration of cortical interneurons into a functional network. Curr Top Dev Biol, 87, 81-118.

Bortone, D. S., Olsen, S. R., \& Scanziani, M. (2014). Translaminar inhibitory cells recruited by layer 6 corticothalamic neurons suppress visual cortex. Neuron, 82(2), 474-485.

Briggs, F. (2010). Organizing principles of cortical layer 6. Frontiers in neural circuits, 4.

Cajal, S. R. (1911). Histologie du syste me nerveux de I'Homme et des verte be s. Maloine (Paris), 2, 891-942.

Cardin, J. A., Carlén, M., Meletis, K., Knoblich, U., Zhang, F., Deisseroth, K., . . Moore, C. I. (2009). Driving fast-spiking cells induces gamma rhythm and controls sensory responses. Nature, 459(7247), 663-667.

Chen, C. C., Abrams, S., Pinhas, A., \& Brumberg, J. C. (2009). Morphological heterogeneity of layer VI neurons in mouse barrel cortex. Journal of Comparative Neurology, 512(6), 726-746. 
Cruikshank, S. J., Urabe, H., Nurmikko, A. V., \& Connors, B. W. (2010). Pathway-specific feedforward circuits between thalamus and neocortex revealed by selective optical stimulation of axons. Neuron, 65(2), 230-245.

Doischer, D., Hosp, J. A., Yanagawa, Y., Obata, K., Jonas, P., Vida, I., \& Bartos, M. (2008). Postnatal differentiation of basket cells from slow to fast signaling devices. Journal of Neuroscience, 28(48), 12956-12968.

Ferrer, I., Fabregues, I., \& Condom, E. (1986). A Golgi study of the sixth layer of the cerebral cortex. I. The lissencephalic brain of Rodentia, Lagomorpha, Insectivora and Chiroptera. Journal of anatomy, 145, 217.

Gabbott, P. L., Dickie, B. G., Vaid, R. R., Headlam, A. J., \& Bacon, S. J. (1997). Local-circuit neurones in the medial prefrontal cortex (areas 25, 32 and 24b) in the rat: morphology and quantitative distribution. Journal of Comparative Neurology, 377(4), 465-499.

Goldberg, E. M., Jeong, H.-Y., Kruglikov, I., Tremblay, R., Lazarenko, R. M., \& Rudy, B. (2010). Rapid developmental maturation of neocortical FS cell intrinsic excitability. Cerebral Cortex, 21(3), 666-682.

Han, Z. S., Buhl, E. H., Lörinczi, Z., \& Somogyi, P. (1993). A High Degree of Spatial Selectivity in the Axonal and Dendritic Domains of Physiologically Identified Local-circuit Neurons in the Dentate Gyms of the Rat Hippocampus. European Journal of Neuroscience, 5(5), 395-410.

Häusser, M., Spruston, N., \& Stuart, G. J. (2000). Diversity and dynamics of dendritic signaling. Science, 290(5492), 739-744.

Hu, H., Gan, J., \& Jonas, P. (2014). Fast-spiking, parvalbumin+ GABAergic interneurons: From cellular design to microcircuit function. Science, 345(6196), 1255263. 
Huang, Z. J., Di Cristo, G., \& Ango, F. (2007). Development of GABA innervation in the cerebral and cerebellar cortices. Nat Rev Neurosci, 8(9), 673-686.

Karube, F., Kubota, Y., \& Kawaguchi, Y. (2004). Axon branching and synaptic bouton phenotypes in GABAergic nonpyramidal cell subtypes. Journal of Neuroscience, 24(12), 2853-2865.

Kawaguchi, Y., \& Kondo, S. (2002). Parvalbumin, somatostatin and cholecystokinin as chemical markers for specific GABAergic interneuron types in the rat frontal cortex. Journal of neurocytology, 31(3), 277-287.

Kumar, P., \& Ohana, O. (2008). Inter-and intralaminar subcircuits of excitatory and inhibitory neurons in layer 6a of the rat barrel cortex. Journal of Neurophysiology, 100(4), 19091922.

Lam, Y.-W., \& Sherman, S. M. (2009). Functional organization of the somatosensory cortical layer 6 feedback to the thalamus. Cerebral Cortex, 20(1), 13-24.

Lewis, D. A., Curley, A. A., Glausier, J. R., \& Volk, D. W. (2012). Cortical parvalbumin interneurons and cognitive dysfunction in schizophrenia. Trends in Neurosciences, 35(1), $57-67$.

Markram, H., Muller, E., Ramaswamy, S., Reimann, M. W., Abdellah, M., Sanchez, C. A., ... Arsever, S. (2015). Reconstruction and simulation of neocortical microcircuitry. Cell, $163(2), 456-492$.

Miller, M. N., Okaty, B. W., Kato, S., \& Nelson, S. B. (2011). Activity-dependent changes in the firing properties of neocortical fast-spiking interneurons in the absence of large changes in gene expression. Developmental neurobiology, 71(1), 62-70. 
O'Leary, J. L. (1941). Structure of the area striata of the cat. Journal of Comparative Neurology, 75(1), 131-164.

Okaty, B. W., Miller, M. N., Sugino, K., Hempel, C. M., \& Nelson, S. B. (2009). Transcriptional and electrophysiological maturation of neocortical fast-spiking GABAergic interneurons. Journal of Neuroscience, 29(21), 7040-7052.

Olsen, S. R., Bortone, D., Adesnik, H., \& Scanziani, M. (2012). Gain control by layer six in cortical circuits of vision. Nature, 483(7387), 47.

Pearce, R. A. (1993). Physiological evidence for two distinct GABA A responses in rat hippocampus. Neuron, 10(2), 189-200.

Pouille, F., \& Scanziani, M. (2001). Enforcement of temporal fidelity in pyramidal cells by somatic feed-forward inhibition. Science, 293(5532), 1159-1163.

Prieto, J. J., \& Winer, J. A. (1999). Layer VI in cat primary auditory cortex: Golgi study and sublaminar origins of projection neurons. Journal of Comparative Neurology, 404(3), 332-358.

Radnikow, G., Qi, G., \& Feldmeyer, D. (2015). Synaptic Microcircuits in the Barrel Cortex Sensorimotor Integration in the Whisker System (pp. 59-108): Springer.

Rall, W. (1967). Distinguishing theoretical synaptic potentials computed for different somadendritic distributions of synaptic input. Journal of Neurophysiology, 30(5), 1138-1168.

Rudy, B., Fishell, G., Lee, S., \& Hjerling-Leffler, J. (2011). Three groups of interneurons account for nearly $100 \%$ of neocortical GABAergic neurons. Developmental neurobiology, 71(1), 45-61.

Rudy, B., \& McBain, C. J. (2001). Kv3 channels: voltage-gated K+ channels designed for highfrequency repetitive firing. Trends in Neurosciences, 24(9), 517-526. 
Somogyi, P., Tamas, G., Lujan, R., \& Buhl, E. H. (1998). Salient features of synaptic organisation in the cerebral cortex. Brain research reviews, 26(2), 113-135.

Thomson, A. M. (2010). Neocortical layer 6, a review. Frontiers in neuroanatomy, 4.

Thomson, A. M., \& Lamy, C. (2007). Functional maps of neocortical local circuitry. Frontiers in neuroscience, 1(1), 19.

Tömböl, T. (1984). Layer VI cells. Cerebral Cortex, 1, 479-519.

Uematsu, M., Hirai, Y., Karube, F., Ebihara, S., Kato, M., Abe, K., . . Yanagawa, Y. (2007). Quantitative chemical composition of cortical GABAergic neurons revealed in transgenic venus-expressing rats. Cerebral Cortex, 18(2), 315-330.

Van Eden, C., \& Uylings, H. (1985). Cytoarchitectonic development of the prefrontal cortex in the rat. Journal of Comparative Neurology, 241(3), 253-267.

Wang, G., Wyskiel, D. R., Yang, W., Wang, Y., Milbern, L. C., Lalanne, T., . . Zhu, J. J. (2015). An optogenetics-and imaging-assisted simultaneous multiple patch-clamp recording system for decoding complex neural circuits. Nature protocols, 10(3), 397-412.

West, D. C., Mercer, A., Kirchhecker, S., Morris, O. T., \& Thomson, A. M. (2005). Layer 6 cortico-thalamic pyramidal cells preferentially innervate interneurons and generate facilitating EPSPs. Cerebral Cortex, 16(2), 200-211.

Wisden, W., Laurie, D. J., Monyer, H., \& Seeburg, P. H. (1992). The distribution of 13 GABAA receptor subunit mRNAs in the rat brain. I. Telencephalon, diencephalon, mesencephalon. Journal of Neuroscience, 12(3), 1040-1062.

Wyskiel, D. R., Larry, T. C., Jiang, X., Wang, G., \& Zhu, J. J. (2016). Analysis of Transsynaptic Attentional Neuronal Circuits with Octuple Patch-Clamp Recordings. Advanced PatchClamp Analysis for Neuroscientists, 139-150. 


\section{Figure legends}

Figure 1. Spiking and passive membrane responses of two reconstructed L6 interneurons and their anatomical reconstructions. A. Responses to hyperpolarizing and depolarizing current injections for a FS (left) and a NFS interneuron (right), top trace: near hyperpolarizing; bottom trace: hyperpolarizing and suprathreshold. B. Morphological reconstructions for the corresponding FS (left) and NFS (right) interneurons. Cell body and dendrites are in lighter blue and axon is in darker blue.

Figure 2. Quantified intrinsic properties of the reconstructed L6 interneurons. A. B-H. Population data set for all L6 examined interneurons. A. Resting membrane potential ( $\left.\mathrm{V}_{\text {rest }}\right)$. B. Membrane decay time constant (tau). C. Input resistance $\left(\mathrm{R}_{\mathrm{in}}\right)$. D. Afterhyperpolarization (AHP). E. Action potential (AP) threshold. F. Action potential (AP) half-width. G. Action potential (AP) amplitude. H. Adaptation index. I. Firing rate. J. The number of action potentials due to increasing depolarizing current injections. Different colors depict individual cells. Stars correspond to FS interneurons. * $\mathrm{p}<0.05 . * * * \mathrm{p}<0.0005$

Figure 3. Cluster analyses of the reconstructed L6 interneurons for visualization (see methods). A. Model-based clustering using the 9 parameters measured. B. Hierarchical clustering. Numbers correspond to individual interneurons. C. K-means clustering with 2 groups proposed.

Figure 4. Synaptic responses in postsynaptic neurons elicited by the stimulation of L6 interneurons. A-B. Current injections in order to elicit single action potentials in a FS interneuron (A) and a NFS interneuron (B) induced uIPSPs in the postsynaptic neurons. Average uIPSP trace 
is shown in black with individual uIPSP traces in gray. Reconstructions of recorded cells are shown to the right. Interneurons are shown in blue while the postsynaptic neurons are shown in grey. Dendrites are in lighter shades; axons are in darker shades.

Figure 5. Morphological analyses of the reconstructed L6 FS and NFS interneurons. A. Plots of the diameter of the somas in the horizontal (left) and vertical (right). B-F. Graphs comparing the number of primary dendrites (B), the Sholl critical values for dendrites (C) and axons (E), and the number of nodes for dendrites(D) and for axons $(F)$.

Figure 6. Anatomical reconstructions and Sholl analyses of FS and a NFS interneuron. A. Membrane and spiking responses and the reconstructions of the FS $\left(\mathrm{A}_{1}\right)$ and NFS $\left(\mathrm{A}_{2}\right)$ interneurons used for Sholl analysis plots. For the FS interneuron, soma and dendrites are in light blue and axon is in dark blue. For the NFS interneuron, soma and dendrites are in light green and axon is in dark green. B-C. Linear Sholl plots of the axons (B) and dendrites (C) of the two L6a examined interneurons.

Figure 7. Examples of the simultaneous patch-clamp system for a FS and a NFS L6 interneuron. $A_{1}$ and $B_{1}$. Recordings showing the synaptic responses of multiple postsynaptic neurons elicited by the stimulation of a single FS (A1) and a single NFS (B1) interneuron. A2 and B2. Anatomical reconstructions of a FS (A1) and a NFS (A2) interneuron and their postsynaptic neurons (1 and 2) with putative contact sites labeled. A2-B2. Interneurons are shown in blue. A. Postsynaptic neurons are shown in black (1) and pink (2). B. Postsynaptic neurons are shown in pink (1) and maroon (2). 
Figure 8. Plots illustrating the relationships between uIPSP properties obtained in postsynaptic neurons and properties obtained through anatomical reconstructions of the presynaptic L6 interneurons. A. uIPSP absolute amplitude verses number of putative contact sites on the postsynaptic neuron $(n=10 ; r=-0.24 ; p>0.05)$. B. uIPSP rise time verses the putative contact site averaged distance from the soma $(n=10 ; r=-0.42 ; p>0.05)$. C. The slope $(\Delta V / \Delta t)$ of the response verses the averaged distance from the soma $(n=10 ; r=-0.15 ; p>0.05)$. D. uIPSP decay time constant verses the averaged distance from the soma $(n=10 ; r=0.65 ; p<0.05)$.

Notes on figures: Portions of some figures were not produced by me. Some points, values and bars may be approximates. 
Table 1. Amplitude and kinetics of uIPSPs in L6

\begin{tabular}{|c|c|c|}
\hline \multirow[b]{2}{*}{ Postsynaptic neuron } & \multicolumn{2}{|c|}{ Presynaptic neuron } \\
\hline & FS & NFS \\
\hline \multicolumn{3}{|l|}{ Amplitude (mV) } \\
\hline All neurons & $-0.84 \pm 0.01(n=2)$ & $-0.87 \pm 0.31(n=10)$ \\
\hline Pyramidal neurons & $-0.83(n=1)$ & $-1.19 \pm 0.37(n=6)$ \\
\hline \multicolumn{3}{|l|}{ Latency (ms) } \\
\hline All neurons & $0.75 \pm 0.35(n=6)$ & $0.68 \pm 0.26(n=8)$ \\
\hline Pyramidal neurons & $0.41 \pm 0.23(\mathrm{n}=2)$ & $0.41 \pm 0.18(n=6)$ \\
\hline \multicolumn{3}{|l|}{ Rise time (ms) } \\
\hline All neurons & $* 3.25 \pm 0.85(\mathrm{n}=2)$ & $* 7.00 \pm 1.31(\mathrm{n}=9)$ \\
\hline Pyramidal neurons & $2.4(\mathrm{n}=1)$ & $6.00 \pm 0.91(n=6)$ \\
\hline \multicolumn{3}{|l|}{ Half-width (ms) } \\
\hline All neurons & $51.3 \pm 32.7(n=2)$ & $63.4 \pm 9.0(\mathrm{n}=10)$ \\
\hline Pyramidal neurons & $18.6(\mathrm{n}=1)$ & $69.7 \pm 10.8(n=6)$ \\
\hline \multicolumn{3}{|l|}{ Decay (ms) } \\
\hline All neurons & $64.7 \pm 45.3(n=2)$ & $68.5 \pm 10.3(\mathrm{n}=10)$ \\
\hline Pyramidal neurons & $2.4(n=1)$ & $74.5 \pm 11.3(n=6)$ \\
\hline \multicolumn{3}{|l|}{ Slope (mV/ms) } \\
\hline All neurons & $-0.22 \pm 0.06(n=2)$ & $-0.17 \pm 0.08(\mathrm{n}=9)$ \\
\hline Pyramidal neurons & $-0.28(\mathrm{n}=1)$ & $-0.22 \pm 0.09(n=6)$ \\
\hline
\end{tabular}

$* p<0.05$ 
Figure 1

A

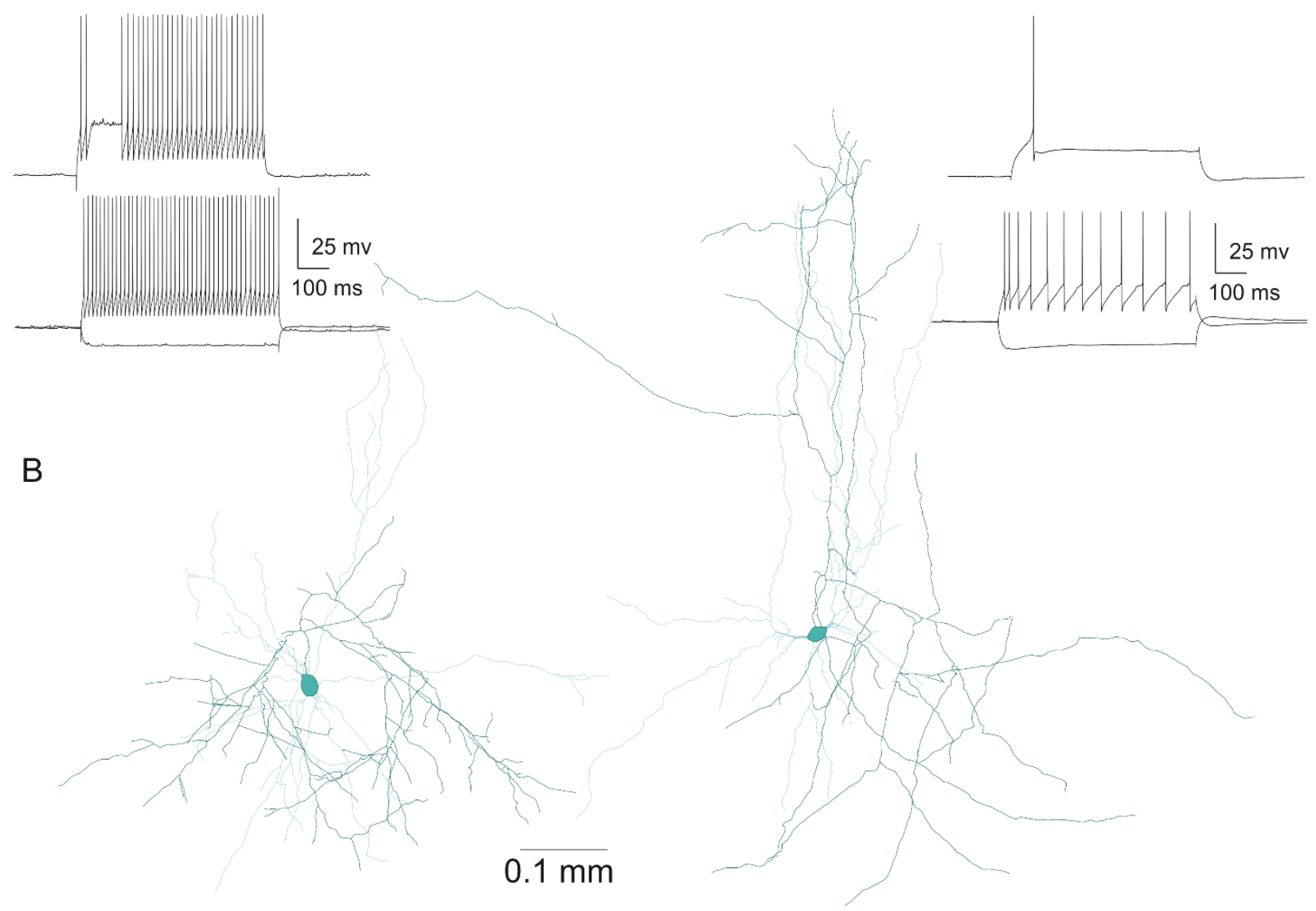


Figure 2
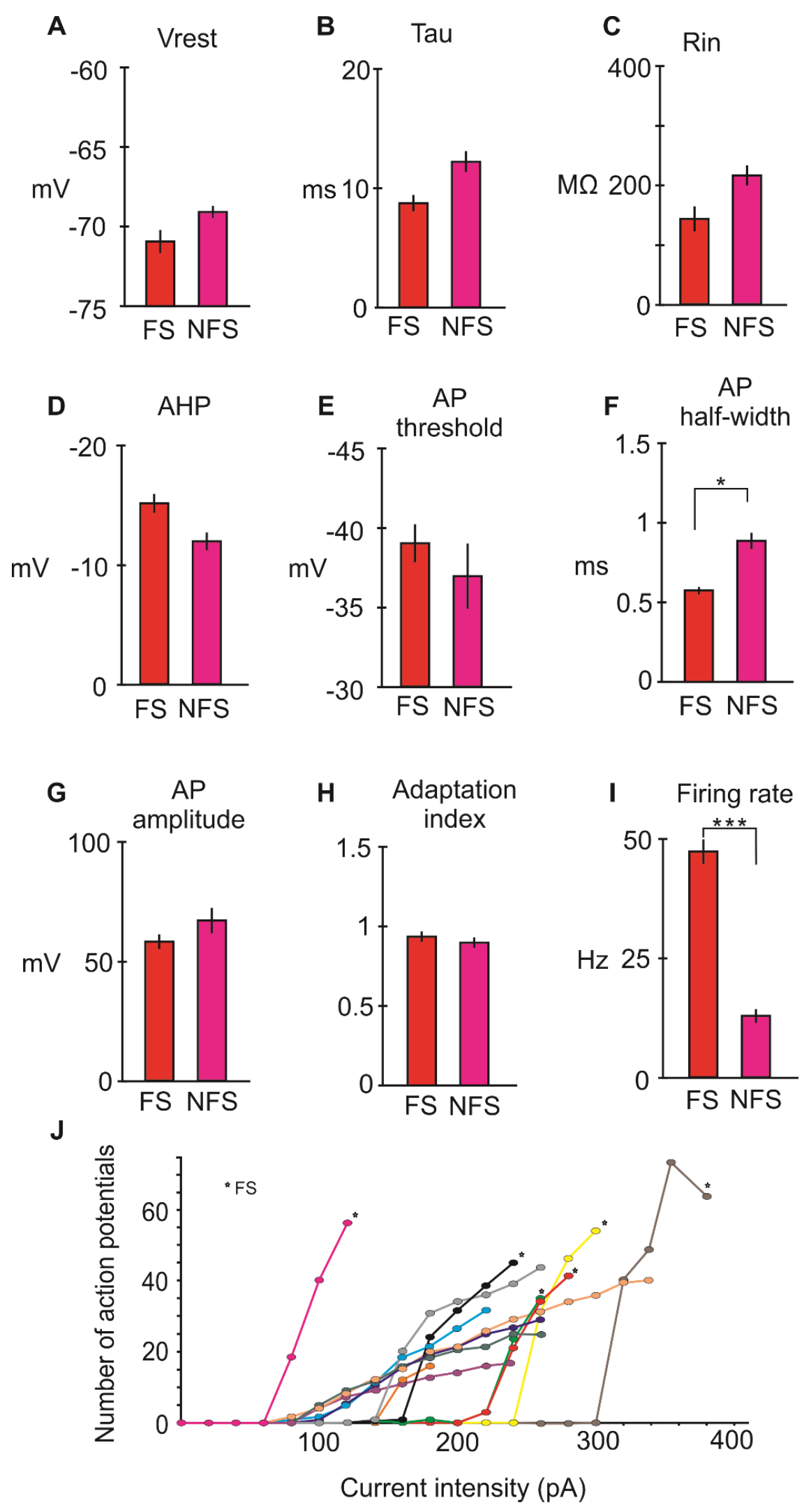


\section{Figure 3}

A.

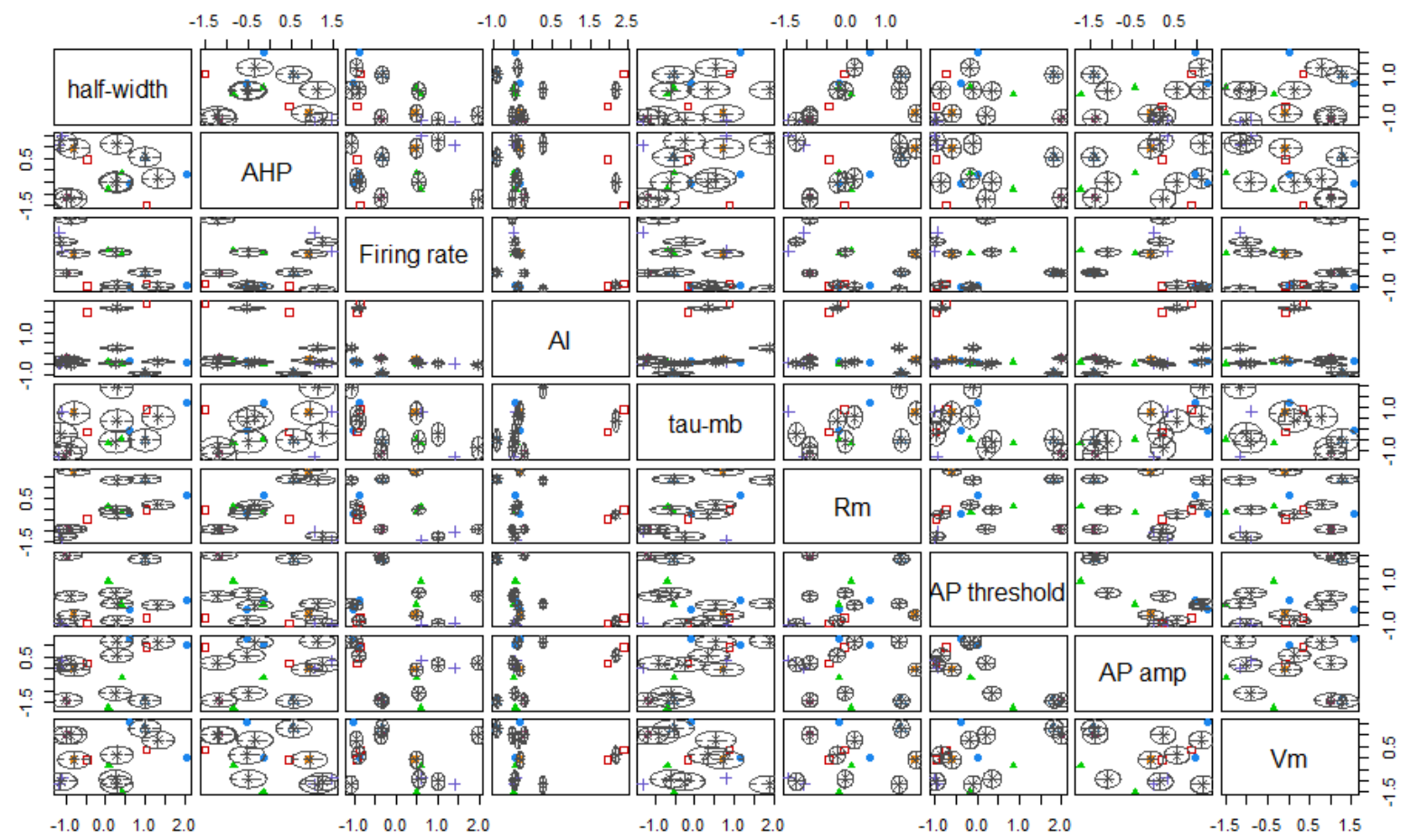


B.

Cluster Dendrogram

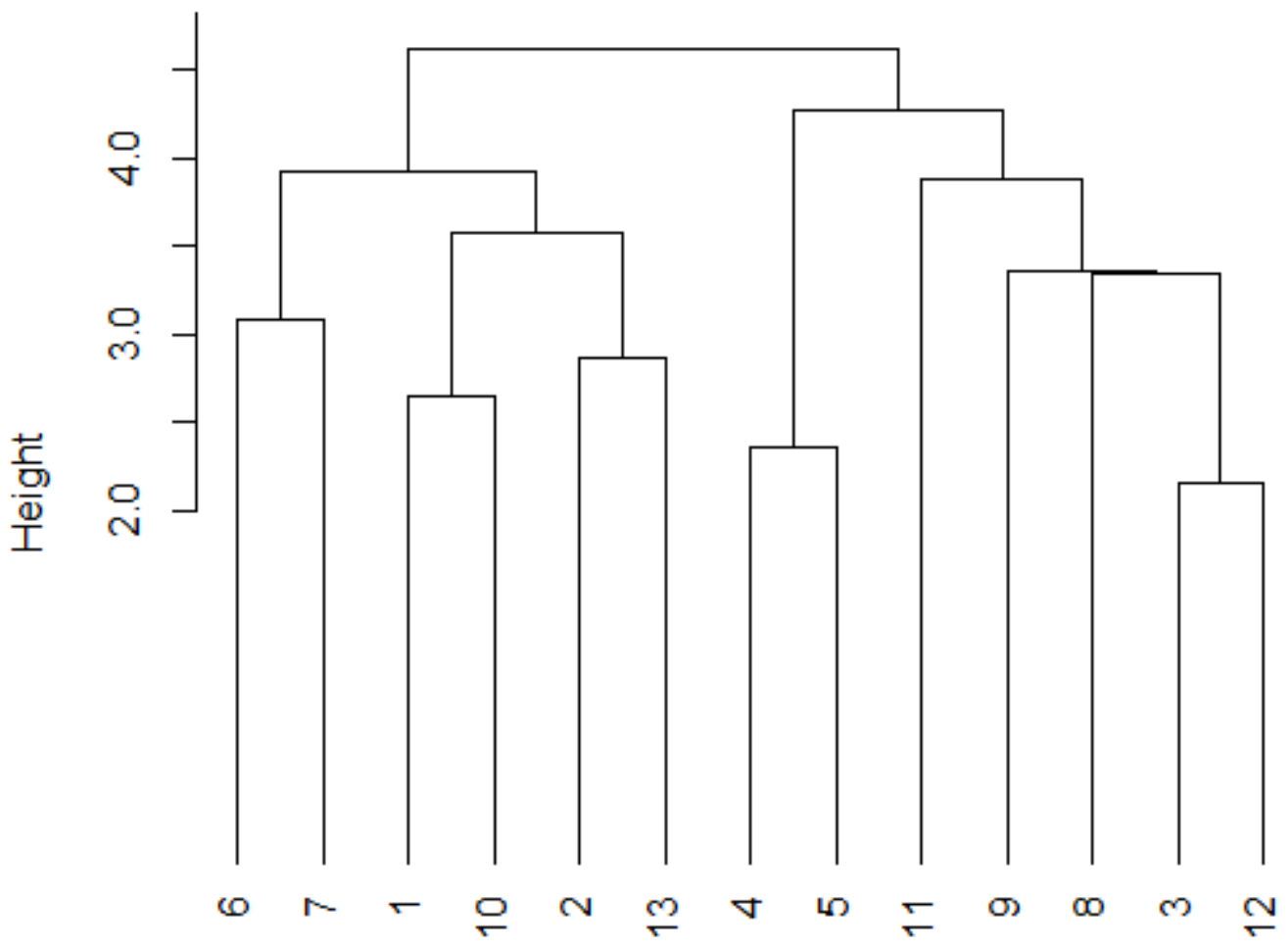

dist(dat)

hclust ( ${ }^{*}$, "average") 
c.

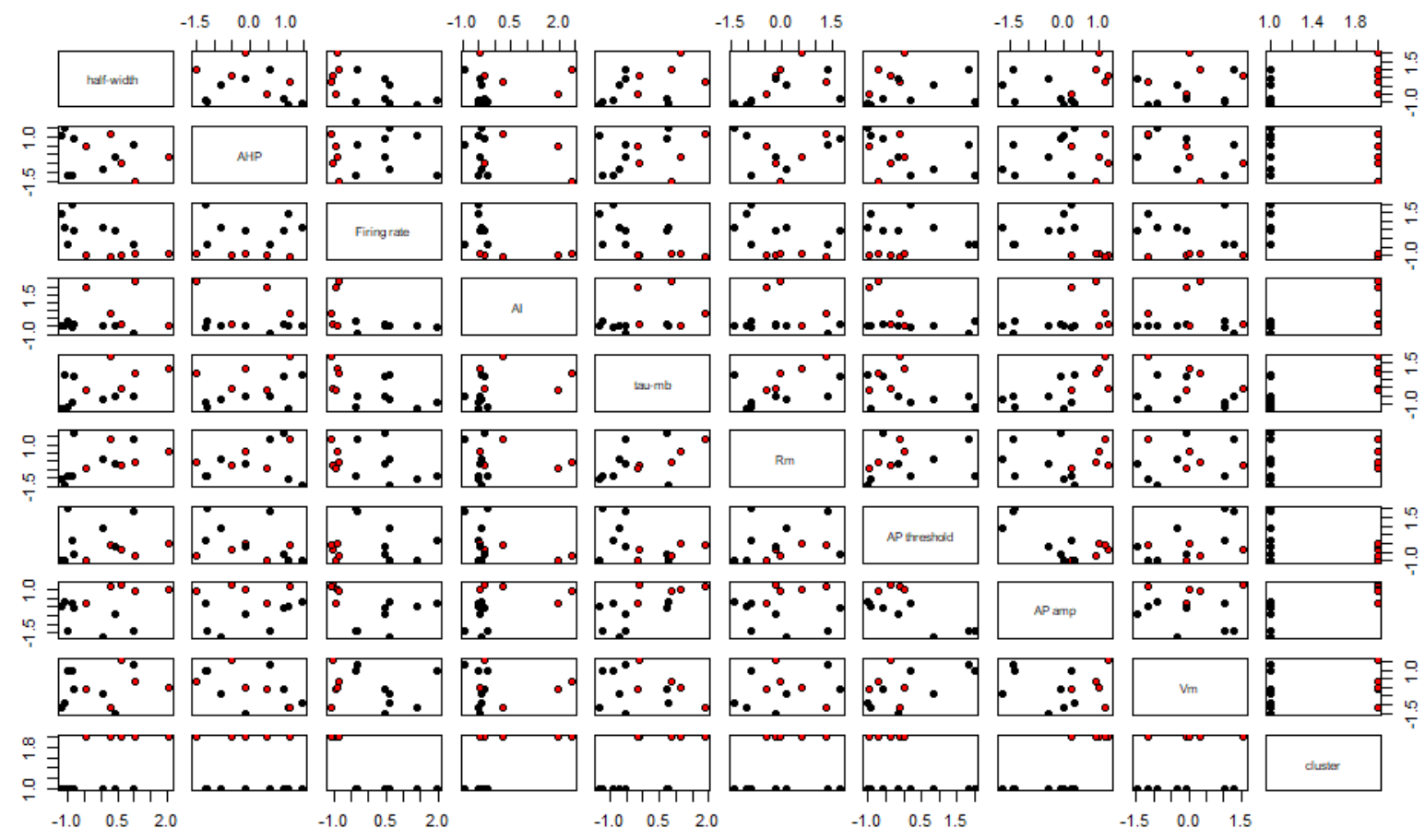




\section{Figure 4}

A
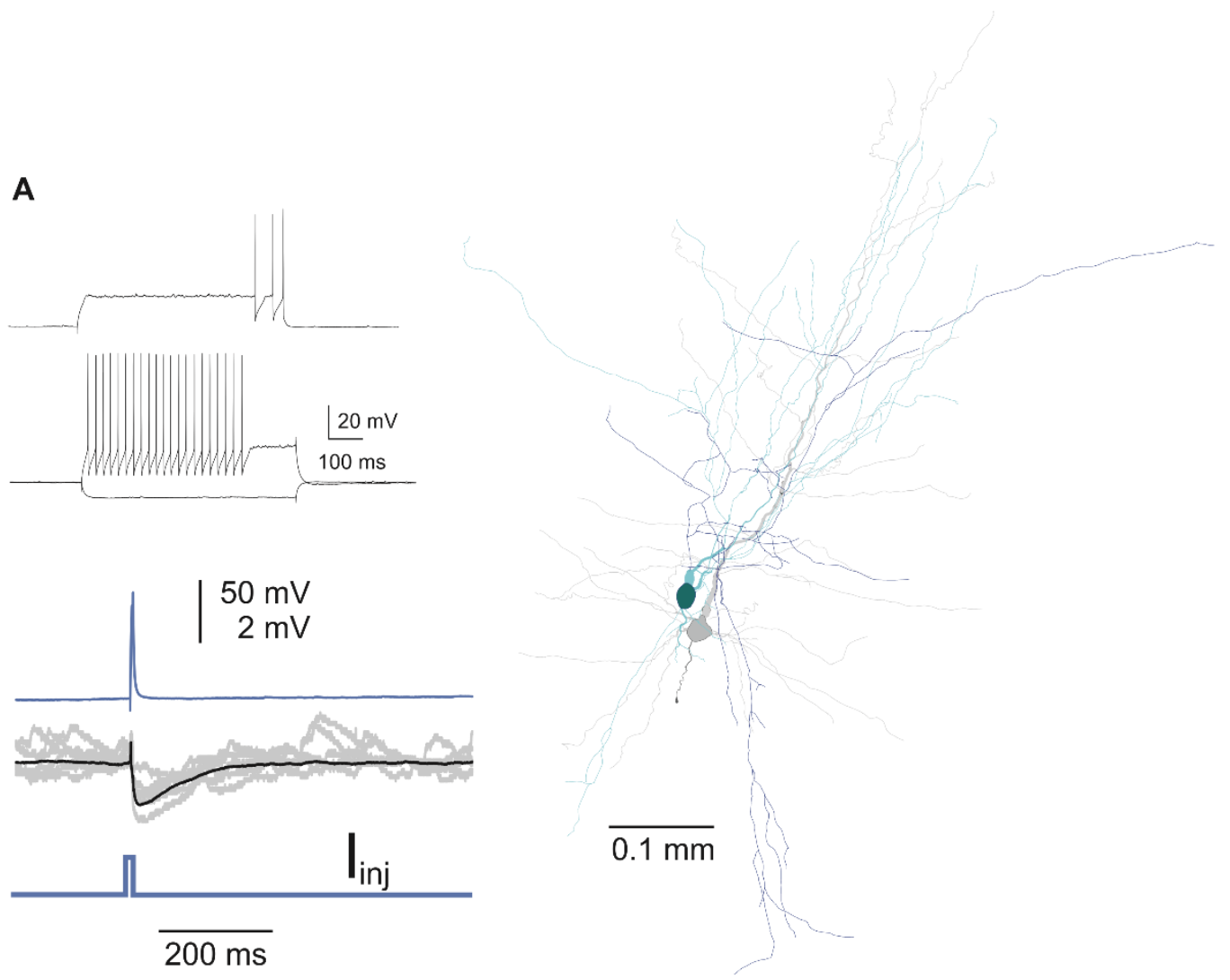

B
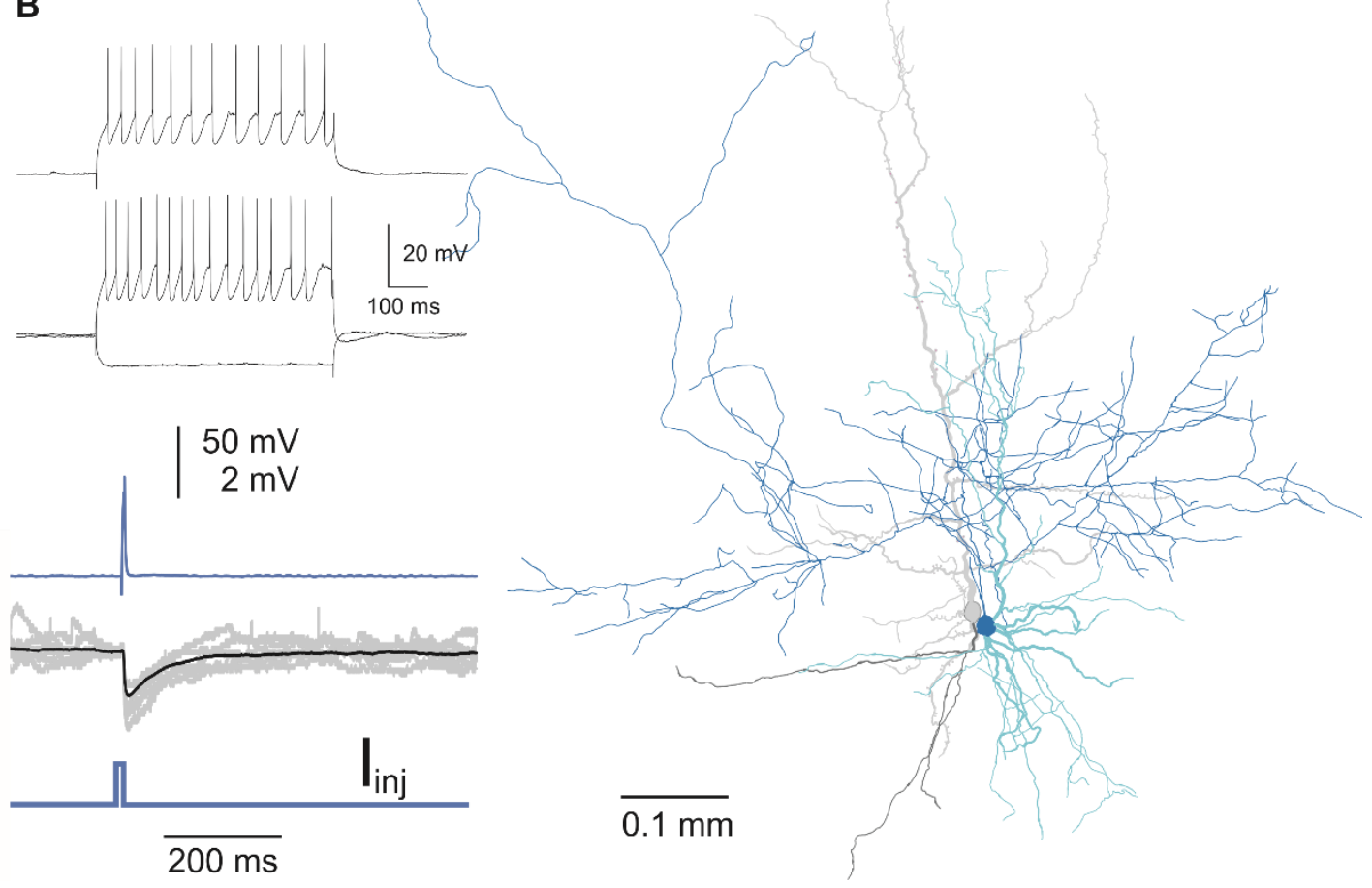
Figure 5
A
OFS
B
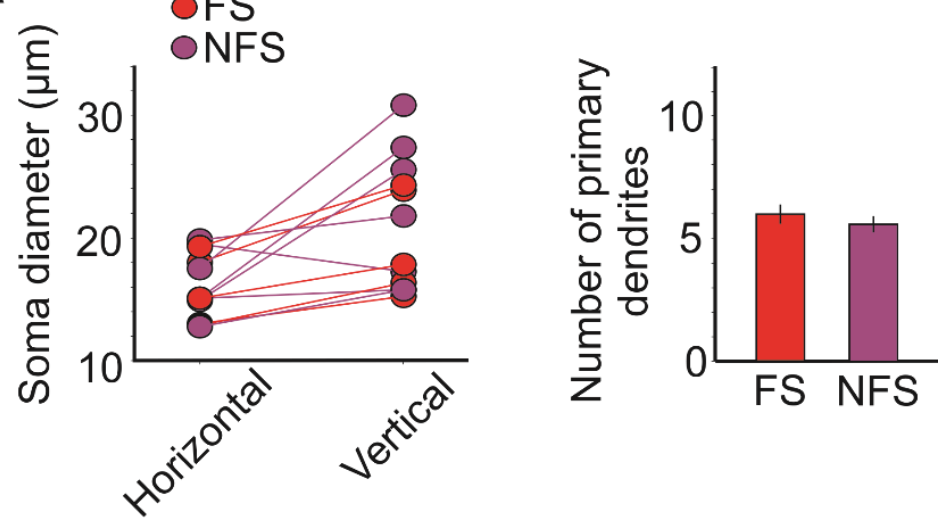
C
D
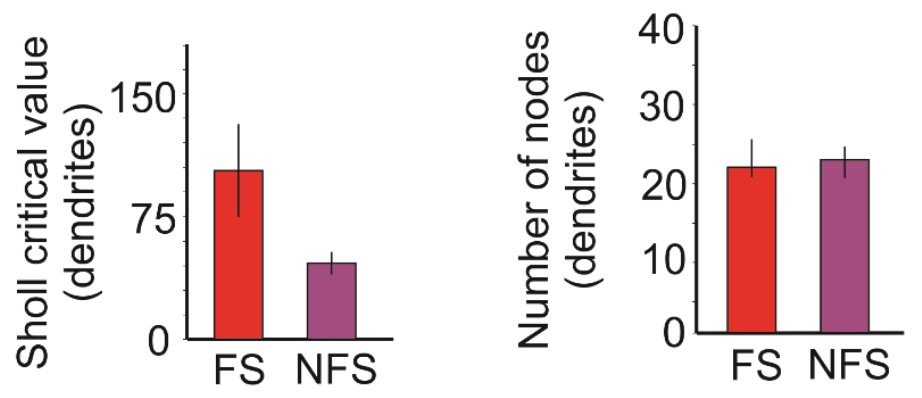
E
F
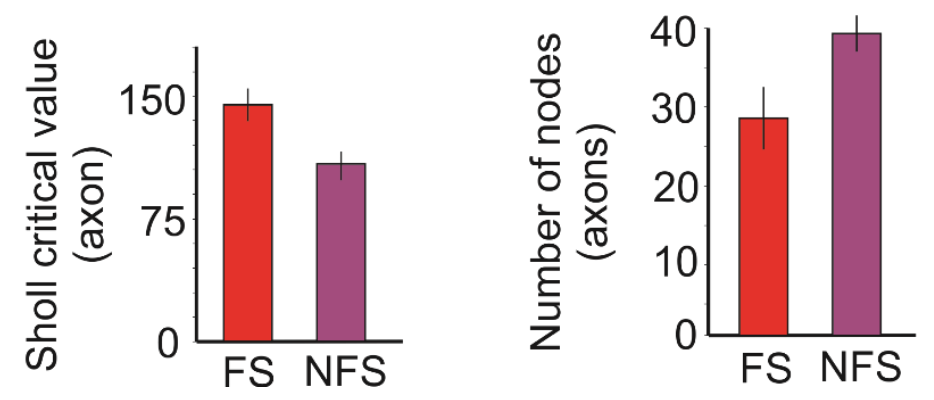


\section{Figure 6}

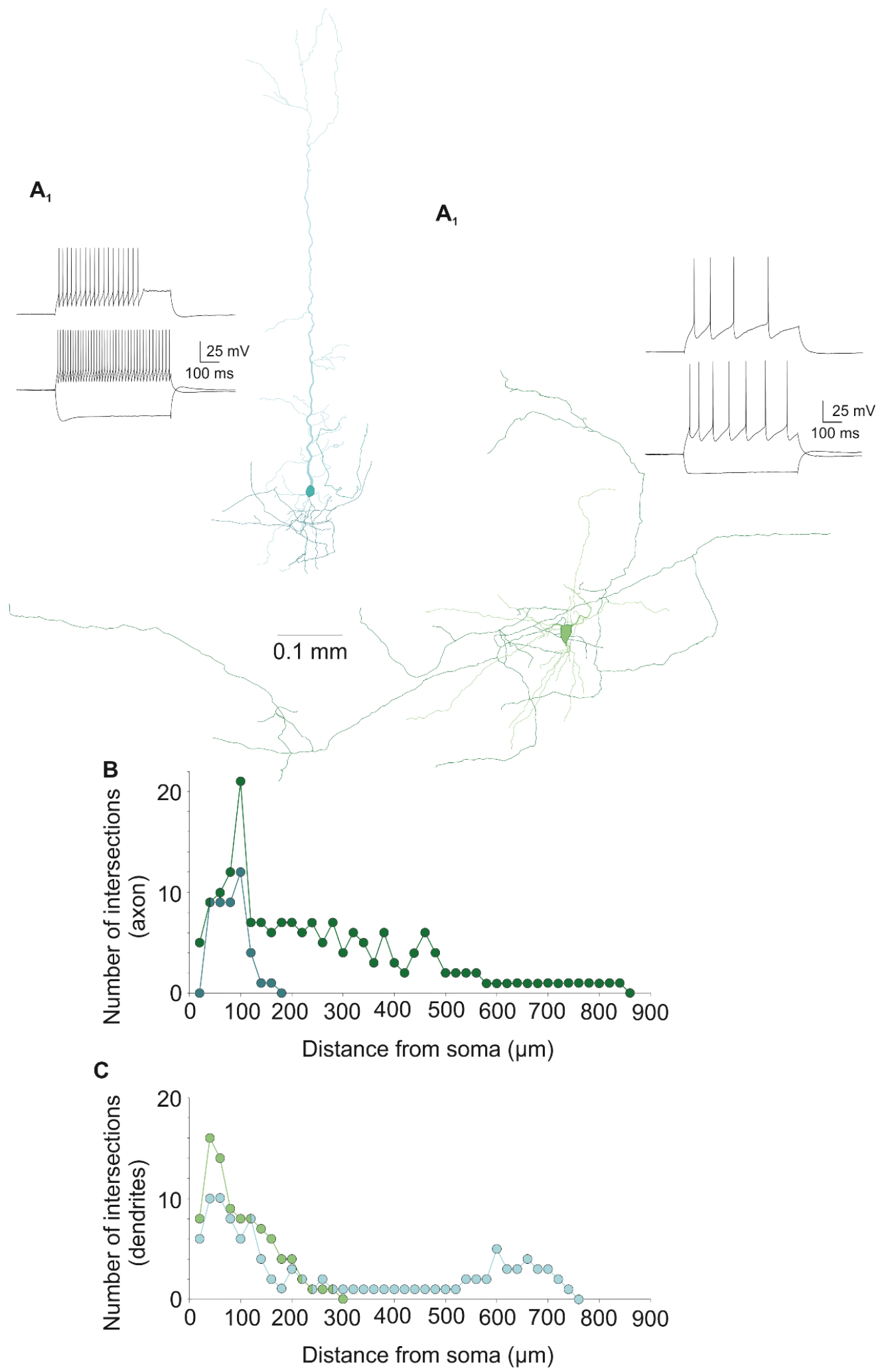


Figure 7

$\mathrm{A}_{1}$

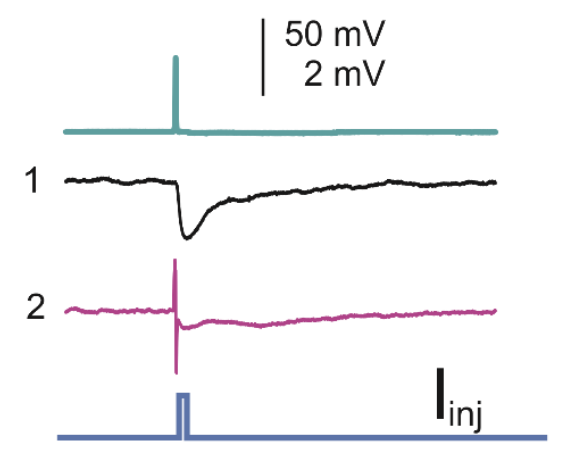

$\mathrm{A}_{2}$

$\mathrm{B}_{1}$

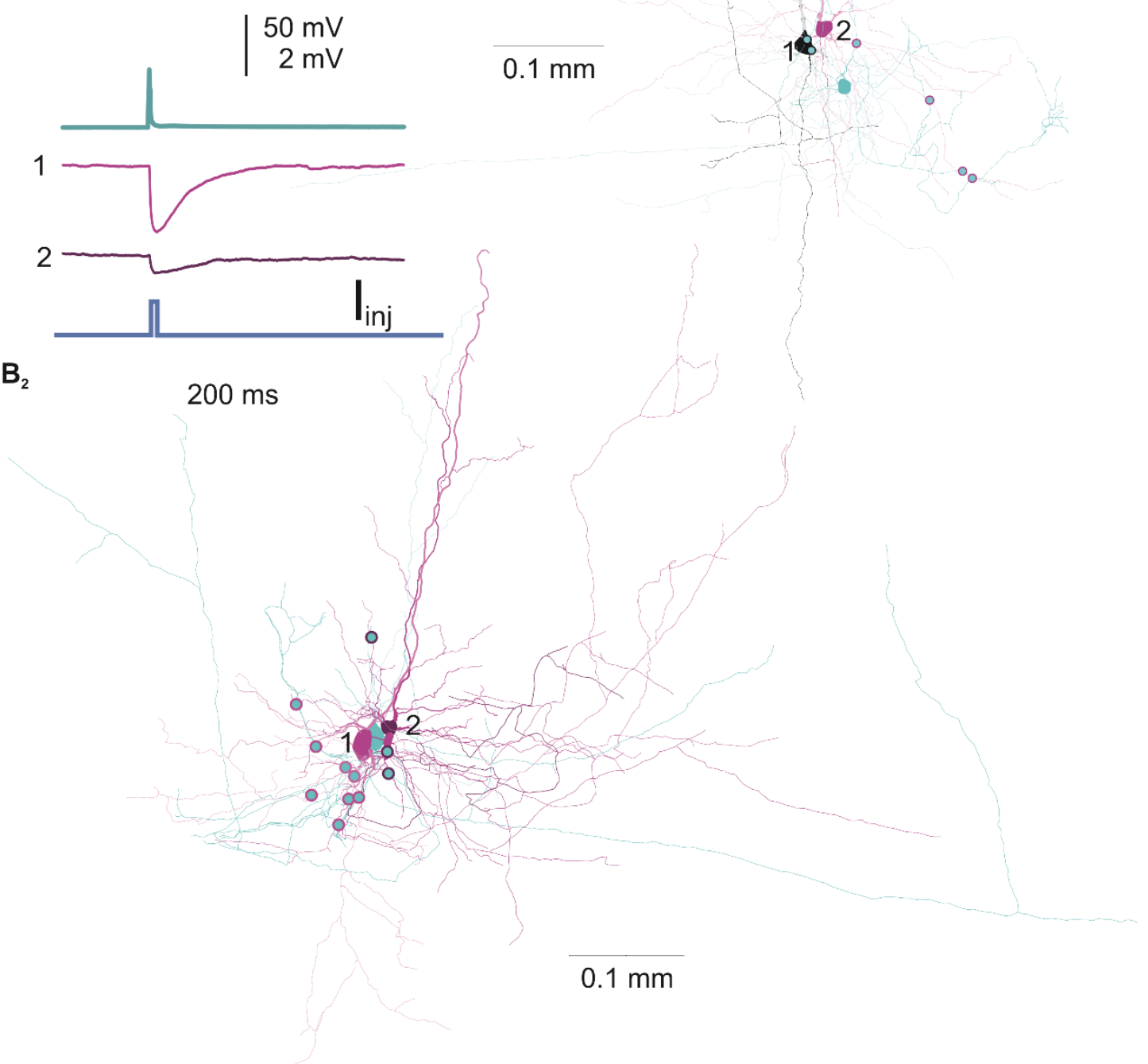


Figure 8

A

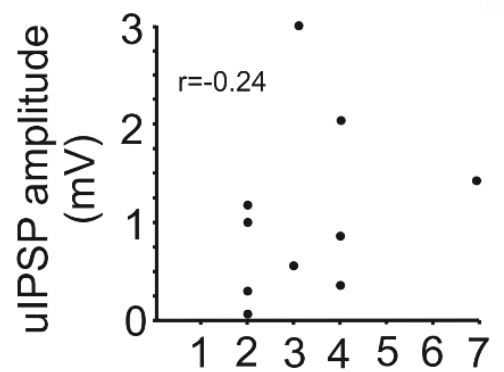

\# of synaptic contacts

C

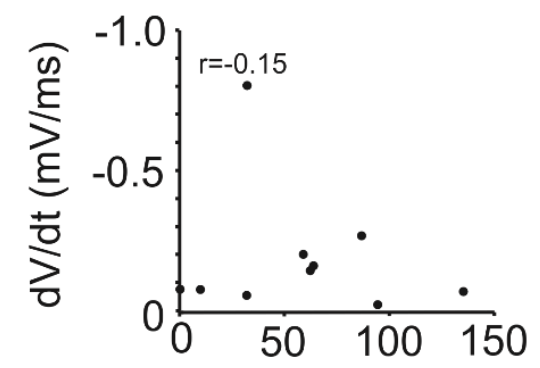

Distance from soma $(\mu \mathrm{m})$
B

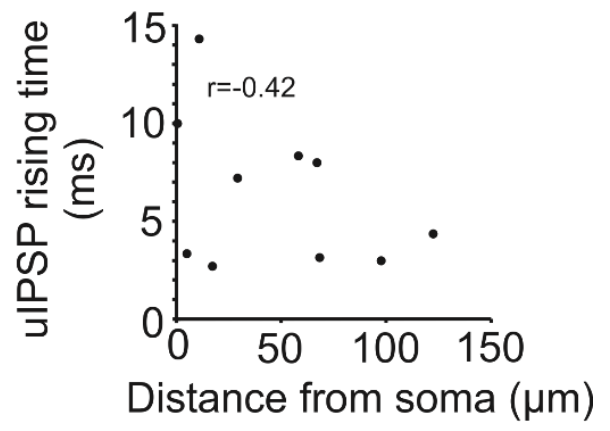

D

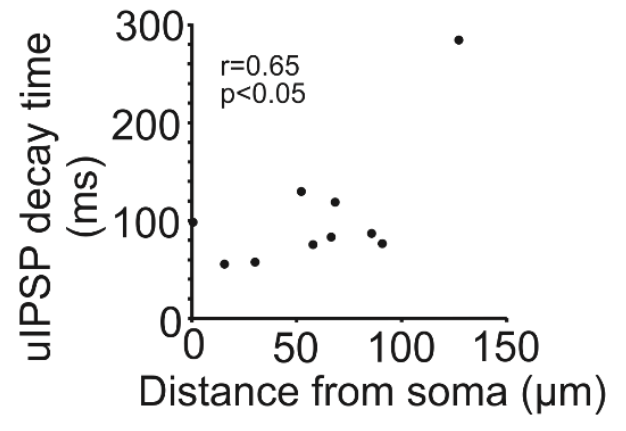




\section{CHAPTER 5}

\section{Discussion}

In this dissertation, I sought to aid in deciphering interneuronal circuitry in L6, the enigmatic cortical layer, with a focus on the role of BCs in L6. In Chapter 1, I began by providing background on L6 and BCs. In Chapters 2 and 3, I described the methods that I helped developed and used to examine L6 interneuronal circuitry. In Chapter 4, I outlined my aims, experiments and results for the project. Overall, my aims were to examine the physiological and anatomical properties of L6 interneurons and then to examine the relationships between these properties. In addition, I sought to determine whether I could identify BCs in L6. Although I did not find any interneurons that formed "baskets" under the light microscope, my analyses led me in another direction. I found that these L6 interneurons could be grouped by their firing rates. I then used these subgroups, FS and NFS interneurons, to examine whether differences could be found in their synaptic physiology and anatomical properties. While my results did not show that these subgroups differed in the majority of properties examined, it may provide a framework for future

studies. My finding that the synaptic response decay positively correlates with the mean distance of putative synaptic contact sites indicates that there is a clear relationship between the synaptic physiological properties and this relationship must be further examined. My results hopefully will aid in elucidating the properties of L6 interneurons and contribute towards understanding the relationships between the physiological and anatomical properties. Overall, my dissertation should have provided a useful background for researchers on L6 and BCs and should have provided insight into the methods used to examine interneuronal circuitry. 
The end goal of this research was to better understand neuronal circuitry in L6. However, many factors must be understood in order to achieve this. To understand how neuronal circuits underlie the processing of brain function, methods must be employed to dissect the circuit. These methods entail identifying the individual components of the circuits and characterizing how they interact. The main individual components of a neuronal circuit are the excitatory neurons (largely pyramidal neurons) and the inhibitory neurons (GABAergic interneurons). How they interact is mainly through synaptic connections. Different methods have been employed to dissect these circuits (Luo, Callaway, \& Svoboda, 2008; Tye \& Deisseroth, 2012; G. Wang et al., 2015). Many factors have made the undertaking of dissecting neuronal circuits challenging. Chief among them is the great diversity in both their individual components and how they respond. The diversity in GABAergic interneurons has been especially recognized, and a lack of understanding of the significance of the diversity has hindered progress (G. A. Ascoli et al., 2008; Javier DeFelipe et al., 2013).

The method to dissect neuronal circuits I helped develop, have described and have employed is simultaneous multiple patch-clamp recording system (G. Wang et al., 2015; Wyskiel, Larry, Jiang, Wang, \& Zhu, 2016). As outlined above, to identify the individual components of the circuit, I examined their intrinsic electrophysiological properties and attempted to recover their morphology. To examine how they interact, I used multiple simultaneous patch-clamp recordings and induce activity in individual components to determine which components are connected, and I then analyzed those connections. I initially concentrated on the role of BCs in L6. To be able to examine L6 BCs as an individual component in the circuit, a clear method for identification of BCs was crucial. Ideally, this method needs to be reproducible and should be 
universally recognized so that insights obtained advance the field. However, a significant number of factors have prevented this. BCs display a wide range of axonal and dendritic arborization patterns (T. Freund, Martin, Smith, \& Somogyi, 1983; Kisvárday, 1992) (Kisvarday, Martin, Whitteridge, \& Somogyi, 1985; Markram et al., 2004; Martin, Somogyi, \& Whitteridge, 1983; Somogyi, Kisvarday, Martin, \& Whitteridge, 1983; Thomson \& Lamy, 2007; Y. Wang, Gupta, Toledo-Rodriguez, Wu, \& Markram, 2002). The axonal arbor can be dense and largely contained within a cortical layer near the soma, or it can be across many cortical layers and span across columns. While a smaller portion of their contacts target somas, the great majority are on dendrites, either on spines or shafts (T. Freund, Maglóczky, Soltesz, \& Somogyi, 1986; Kisvarday, Martin, Somogyi, \& Friedlander, 1987; Kisvarday et al., 1985; Somogyi et al., 1983). However, a BC may not synapse at the soma of a connected pyramidal neuron (Kisvarday et al., 1987; Kubota et al., 2015; Tamás, Somogyi, \& Buhl, 1998; Y. Wang et al., 2002). If an investigator is examining this connected pair, it may lead to misidentifying the presynaptic cell. BCs are also diverse in their intrinsic electrophysiological properties, some of which have critical roles in the circuit (Tremblay, Lee, \& Rudy, 2016). Biochemically, BCs are also heterogeneous. Differences in both connectivity and function are found in proposed subdivisions (PV BCs verses $\mathrm{CCK} \mathrm{BCs}$ ) that are highly diverse themselves; for example, $\mathrm{PV} \mathrm{BCs}$ are diverse in morphology, including axonal arborization, in their physiology and their biochemical content (Yasuo Kawaguchi \& Kubota, 1997; Y Kawaguchi \& Kubota, 1998; Kubota \& Kawaguchi, 1997). In addition, differences are found where the synapse is made on the postsynaptic neuron (at the soma verses at the dendrite), including differences in function (Armstrong \& Soltesz, 2012; Bartos \& Elgueta, 2012; T. F. Freund \& Katona, 2007; Tremblay et al., 2016). While FS BCs in most of the cortex typically express PV, studies to determine if this correlation can be 
observed in L6 are not clear. While the expression of PV largely correlates to L6 FS interneurons (Perrenoud, Rossier, Geoffroy, Vitalis, \& Gallopin, 2012), studies correlating PV and the morphologies have not been clear (Arzt, Sakmann, \& Meyer, 2017; Perrenoud et al., 2012). In addition, whether it corresponds to BCs has not been shown.

The morphologies of GABAergic interneurons, including BCs, have been classically used to for identification starting with Ramón y Cajal (Cajal, 1911; Ramón, 1899; Ramon \& Cajal, 1904). Research spanning the decades that followed have led to different classification schemes, including the use of their physiology and biochemical content (Cauli et al., 1997; Gupta, Wang, \& Markram, 2000). The lack of an unequivocal identification method to classify interneurons became apparent. The establishment of the Petilla terminology was proposed to help form a more consistent system (Ascoli, Alonso-Nanclares, Anderson, \& German Barrionuevo, 2008). The use of a "gardener's" approach to classify interneurons based on their morphologies was proposed; however, the "study empirically and quantitatively demonstrates that the gardener's approach to neuron classification is untenable at this time and confirms the impression that different investigators use their own, mutually inconsistent schemes for classifying neurons based on morphological criteria" (Javier DeFelipe et al., 2013).

The use of multiple approaches to identify GABAergic interneurons has been advocated by some researchers in the field (Barth et al., 2016). For BCs, this should be standard. However, due to the many different ways in identifying BCs, a BC identified by one research group, may not correlate with a BC identified by another. Therefore, a consensus needs to be made regarding these methods. Furthermore, either BCs need a better definition or these cells need to be divided 
into distinct accepted subgroups. Overall, a better understanding of what should define a subtype is needed, which should better correlate with its role in neuronal circuits.

The criteria I used in my study to define BCs should be revised. This criteria was the identification of cells that synapse in a "basket" formation around the postsynaptic neuron observed under the light microscope. The formation of "baskets" is typically a result of multiple BCs synapses on the soma of a postsynaptic neuron (J DeFelipe, Hendry, \& Jones, 1986; MarinPadilla, 1969; Somogyi et al., 1983; Somogyi \& Soltesz, 1986; White \& Keller, 1989). Therefore, examining a single interneuron and its postsynaptic formations will not meet the criteria. A criteria to identify BCs based on the analysis of their perisomatic synapses using electron microscopy has been established by many other researchers (T. Freund et al., 1986; Jones, 1984; Kisvárday, 1992; Somogyi \& Soltesz, 1986). Therefore, electron microscopy should be employed. However, BCs form two functionally distinct subgroups that are either FS or NFS (Y Kawaguchi \& Kubota, 1998). This necessitates the use of the physiology in addition to the morphology when establishing subgroups. Further specificity, such one using a non-overlapping neurochemical marker, is needed. This specificity is crucial to identifying that component of the circuit, which can be used for targeting and monitoring, manipulating, and modeling to give us a better understanding of its role in the circuit. 


\section{References}

Armstrong, C., \& Soltesz, I. (2012). Basket cell dichotomy in microcircuit function. The Journal of Physiology, 590(4), 683-694.

Arzt, M., Sakmann, B., \& Meyer, H. S. (2017). Anatomical Correlates of Local, Translaminar, and Transcolumnar Inhibition by Layer 6 GABAergic Interneurons in Somatosensory Cortex. Cerebral Cortex, 1-12.

Ascoli, Alonso-Nanclares, Anderson, \& German Barrionuevo, R. B.-P., Andreas Burkhalter, György Buzsáki, Bruno Cauli, Javier DeFelipe, Alfonso Fairén, Dirk Feldmeyer, Gord Fishell, Yves Fregnac, Tamas F. Freund, Daniel Gardner, Esther P. Gardner, Jesse H. Goldberg, Moritz Helmstaedter, Shaul Hestrin, Fuyuki Karube, Zoltán F. Kisvárday, Bertrand Lambolez, David A. Lewis, Oscar Marin, Henry Markram, Alberto Muñoz, Adam Packer, Carl C. H. Petersen, Kathleen S. Rockland, Jean Rossier, Bernardo Rudy, Peter Somogyi, Jochen F. Staiger, Gabor Tamas, Alex M. Thomson, Maria ToledoRodriguez, Yun Wang, David C. West \& Rafael Yuste. (2008). Petilla terminology: nomenclature of features of GABAergic interneurons of the cerebral cortex. [10.1038/nrn2402]. Nat Rev Neurosci, 9(7), 557-568. doi: http://www.nature.com/nrn/journal/v9/n7/suppinfo/nrn2402_S1.html

Ascoli, G. A., Alonso-Nanclares, L., Anderson, S. A., Barrionuevo, G., Benavides-Piccione, R., Burkhalter, A., ... Yuste, R. (2008). Petilla terminology: nomenclature of features of GABAergic interneurons of the cerebral cortex. Nat Rev Neurosci, 9(7), 557-568. 
Barth, A. L., Andreas Burkhalter, Edward M. Callaway, Barry W. Connors, Bruno Cauli, Javier DeFelipe, ... Yuste, R. (2016). Comment on "Principles of connectivity among morphologically defined cell types in adult neocortex". Science, 353(6304), 1108-1108.

Bartos, M., \& Elgueta, C. (2012). Functional characteristics of parvalbumin-and cholecystokininexpressing basket cells. The Journal of Physiology, 590(4), 669-681.

Cajal, S. R. (1911). Histologie du syste me nerveux de I'Homme et des verte be s. Maloine (Paris), 2, 891-942.

Cauli, B., Audinat, E., Lambolez, B., Angulo, M. C., Ropert, N., Tsuzuki, K., . . Rossier, J. (1997). Molecular and physiological diversity of cortical nonpyramidal cells. Journal of Neuroscience, 17(10), 3894-3906.

DeFelipe, J., Hendry, S., \& Jones, E. (1986). A correlative electron microscopic study of basket cells and large GABAergic neurons in the monkey sensory-motor cortex. Neuroscience, 17(4), 991-1009.

DeFelipe, J., López-Cruz, P. L., Benavides-Piccione, R., Bielza, C., Larrañaga, P., Anderson, S., . . Feldmeyer, D. (2013). New insights into the classification and nomenclature of cortical GABAergic interneurons. Nature reviews. Neuroscience, 14(3), 202.

Freund, T., Maglóczky, Z., Soltesz, I., \& Somogyi, P. (1986). Synaptic connections, axonal and dendritic patterns of neurons immunoreactive for cholecystokinin in the visual cortex of the cat. Neuroscience, 19(4), 1133-1159.

Freund, T., Martin, K., Smith, A., \& Somogyi, P. (1983). Glutamate decarboxylaseimmunoreactive terminals of Golgi-impregnated axoaxonic cells and of presumed basket cells in synaptic contact with pyramidal neurons of the cat's visual cortex. Journal of Comparative Neurology, 221(3), 263-278. 
Freund, T. F., \& Katona, I. (2007). Perisomatic inhibition. Neuron, 56(1), 33-42.

Gupta, A., Wang, Y., \& Markram, H. (2000). Organizing principles for a diversity of GABAergic interneurons and synapses in the neocortex. Science, 287(5451), 273-278.

Jones, E. H., SHC. (1984). Basket cells. In Peters, A; Jones, EG. Cerebral cortex. Vol. 1 Cellular components of the cerebral cortex. 309-336.

Kawaguchi, Y., \& Kubota, Y. (1997). GABAergic cell subtypes and their synaptic connections in rat frontal cortex. Cerebral cortex (New York, NY: 1991), 7(6), 476-486.

Kawaguchi, Y., \& Kubota, Y. (1998). Neurochemical features and synaptic connections of large physiologically-identified GABAergic cells in the rat frontal cortex. Neuroscience, 85(3), $677-701$.

Kisvarday, Z., Martin, K., Somogyi, P., \& Friedlander, M. (1987). Evidence for interlaminar inhibitory circuits in the striate cortex of the cat. Journal of Comparative Neurology, 260(1), 1-19.

Kisvarday, Z., Martin, K., Whitteridge, D., \& Somogyi, P. (1985). Synaptic connections of intracellularly filled clutch cells: a type of small basket cell in the visual cortex of the cat. Journal of Comparative Neurology, 241(2), 111-137.

Kisvárday, Z. F. (1992). GABAergic networks of basket cells in the visual cortex. Progress in brain research, 90, 385-405.

Kubota, Y., \& Kawaguchi, Y. (1997). Two distinct subgroups of cholecystokininimmunoreactive cortical interneurons. Brain research, 752(1), 175-183.

Kubota, Y., Kondo, S., Nomura, M., Hatada, S., Yamaguchi, N., Mohamed, A. A., . . Kawaguchi, Y. (2015). Functional effects of distinct innervation styles of pyramidal cells by fast spiking cortical interneurons. elife, 4, e07919. 
Luo, L., Callaway, E. M., \& Svoboda, K. (2008). Genetic dissection of neural circuits. Neuron, 57(5), 634-660.

Marin-Padilla, M. (1969). Origin of the pericellular baskets of the pyramidal cells of the human motor cortex: a Golgi study. Brain research, 14(3), 633-646.

Markram, H., Toledo-Rodriguez, M., Wang, Y., Gupta, A., Silberberg, G., \& Wu, C. (2004). Interneurons of the neocortical inhibitory system. Nature reviews. Neuroscience, 5(10), 793.

Martin, K., Somogyi, P., \& Whitteridge, D. (1983). Physiological and morphological properties of identified basket cells in the cat's visual cortex. Experimental brain research, 50(2), 193-200.

Perrenoud, Q., Rossier, J., Geoffroy, H., Vitalis, T., \& Gallopin, T. (2012). Diversity of GABAergic interneurons in layer VIa and VIb of mouse barrel cortex. Cerebral Cortex, bhs032.

Ramón, S. (1899). Textura del sistema nervioso del hombre y de los vertebrados (Histology of the Nervous System of Man and Vertebrates). (Vol. 1): N. Moya.

Ramon, Y., \& Cajal, S. (1904). Textura del Sistema Nervioso del Hombre y de los Vertebrados (Vol. 2): Madrid Nicolas Moya.

Somogyi, P., Kisvarday, Z., Martin, K., \& Whitteridge, D. (1983). Synaptic connections of morphologically identified and physiologically characterized large basket cells in the striate cortex of cat. Neuroscience, 10(2), 261-294.

Somogyi, P., \& Soltesz, I. (1986). Immunogold demonstration of GABA in synaptic terminals of intracellularly recorded, horseradish peroxidase-filled basket cells and clutch cells in the cat's visual cortex. Neuroscience, 19(4), 1051-1065. 
Tamás, G., Somogyi, P., \& Buhl, E. H. (1998). Differentially interconnected networks of GABAergic interneurons in the visual cortex of the cat. Journal of Neuroscience, 18(11), 4255-4270.

Thomson, A. M., \& Lamy, C. (2007). Functional maps of neocortical local circuitry. Frontiers in neuroscience, 1(1), 19.

Tremblay, R., Lee, S., \& Rudy, B. (2016). GABAergic interneurons in the neocortex: from cellular properties to circuits. Neuron, 91(2), 260-292.

Tye, K. M., \& Deisseroth, K. (2012). Optogenetic investigation of neural circuits underlying brain disease in animal models. Nature Reviews Neuroscience, 13(4), 251-266.

Wang, G., Wyskiel, D. R., Yang, W., Wang, Y., Milbern, L. C., Lalanne, T., . . Zhu, J. J. (2015). An optogenetics-and imaging-assisted simultaneous multiple patch-clamp recording system for decoding complex neural circuits. Nature protocols, 10(3), 397-412.

Wang, Y., Gupta, A., Toledo-Rodriguez, M., Wu, C. Z., \& Markram, H. (2002). Anatomical, physiological, molecular and circuit properties of nest basket cells in the developing somatosensory cortex. Cerebral Cortex, 12(4), 395-410.

White, E. L., \& Keller, A. (1989). Cortical circuits: synaptic organization of the cerebral cortex: structure, function, and theory: Birkhäuser Boston.

Wyskiel, D. R., Larry, T. C., Jiang, X., Wang, G., \& Zhu, J. J. (2016). Analysis of Transsynaptic Attentional Neuronal Circuits with Octuple Patch-Clamp Recordings. Advanced PatchClamp Analysis for Neuroscientists, 139-150. 